\title{
On the design of lubricant free piston compressors
}

Paweł Owczarek

17.09.2010 
This research was carried out under the project name 'Design rules for close tolerance lubricant free piston compressors', which was financially supported by the 'Technologiestichting STW'. The financial support of STW is greatly acknowledged.

Samenstelling van de promotiecommissie:

voorzitter en secretaris:

Prof. dr. F. Eising Universiteit Twente

promotor:

Prof. dr. ir. J. Huétink Universiteit Twente

assistent promotor:

Dr. ir. H.J.M. Geijselaers Universiteit Twente

leden:

Prof. dr hab. inż. M. Gawliński Wrocław University of Technology

Ir. E. Haenen

Stirling Cryogenics BV

Dr. ir. J.B.W. Kok

Universiteit Twente

Prof. dr. ir. W.A. Poelman Universiteit Twente

Dr. ir. M.B. de Rooij Universiteit Twente

ISBN 978-90-365-3077-4

1st printing August 2010

Keywords: piston compressor, oil-free, lifetime, efficiency, coatings, gas lubrication

This thesis was prepared with $\mathrm{H}_{\mathrm{E}} \mathrm{X}$ by the author and printed by PrintPartners Ipskamp, Enschede, from an electronic document.

Copyright (c) 2010 by P. Owczarek, Enschede, The Netherlands

All rights reserved. No part of this publication may be reproduced, stored in a retrieval system, or transmitted in any form or by any means, electronic, mechanical, photocopying, recording or otherwise, without prior written permission of the copyright holder. 


\title{
ON THE DESIGN OF LUBRICANT FREE PISTON COMPRESSORS
}

\author{
PROEFSCHRIFT
}

\author{
ter verkrijging van \\ de graad van doctor aan de Universiteit Twente, \\ op gezag van de rector magnificus, \\ prof.dr. H. Brinksma, \\ volgens besluit van het College voor Promoties \\ in het openbaar te verdedigen \\ op vrijdag 17 september 2010 om 13.15 uur
}

door

Paweł Owczarek

geboren op 28 maart 1975

Turek, Poland 
Dit proefschrift is goedgekeurd door de promotor:

Prof. dr. ir. J. Huétink

en de assistent promotor:

Dr. ir. H.J.M. Geijselaers 
for Iza, Kinga and Dominika 



\section{Summary}

This thesis describes the development on long lifetime and an efficient piston compressor operating in a clean environment where oil lubrication must be excluded. Particularly in cooling systems including cryocoolers the presence of oil is a well known problem. A growing number of applications of localized gas liquefaction stations for gas storage and transportation drives the development on cryogenic cooling. At the moment, for those applications, Stirling cooling machines are the most developed technology to generate temperatures in the range of $77 \mathrm{~K}$. However, the lifetime, especially those units above $20 \mathrm{~W}$ cooling power at $80 \mathrm{~K}$ is not satisfactory. Surface wear and gas leakage dominate the compressor's performance. A proper material combination giving minimum surface wear is therefore needed. From our experimental work it follows that protecting the piston surface with DLC coatings, friction and wear can be significantly lowered. Moreover, the piston/cylinder clearance must be narrowed to reduce the power loss due to gas leakage. This, however, leads inherently to the risk of seizure, if the piston/cylinder assembly is not properly designed. An alternative solution has been investigated where the piston is designed as self-(gas)lubricated. Low or zero wear rate and virtually no friction are the main advantages ensuring long lifetime.

No oil lubricant and tight piston/cylinder fit leads to higher demands with respect to design specifications. The engineer is often confronted with various effects that occur during operation, e.g. tribology, material deformation, heat transfer, fluid flow. An accurate prediction of those processes is important to be able to analyze any newly invented design. Two numerical models were employed. For the analysis of heat transfer and distortions of the piston cylinder assembly, a FEM model was used. A proposed new piston design was analyzed showing advantages over conventional design. For the dynamic analysis of a gas lubricated ringless piston an FDM model was developed. Both models proved to be effective numerical tools for design verification. Those models can provide guidelines to achieve an optimal design. For validation purposes, a test set-up was designed to simulate operating conditions of a typical piston compressor. The feasibility of novel design aspects and the performance of selected materials can also be tested and demonstrated on this set-up. 



\section{Samenvatting}

Dit proefschrift beschrijft de invloed op de levensduur en op de efficiëntie van zuigercompressoren die gebruikt worden in een schone omgeving, waar oliesmering moet worden uitgesloten. Vooral in systemen met cryogene koelers is de aanwezigheid van olie een bekend probleem. Het groeiende aantal vloeibaar gasinstallaties met gasopslag en -transport, vraagt om cryogene koeling. Op dit moment zijn voor deze toepassingen de Stirling koelers de meest gangbare om temperaturen in de buurt van $77 \mathrm{~K}$ te bereiken. Echter de levensduur voor met name eenheden met een koelend vermogen groter dan $20 \mathrm{~W}$ bij $80 \mathrm{~K}$ is niet voldoende. Effecten zoals oppervlakteslijtage en gaslekkage beïnvloeden de prestaties van deze compressoren. Een geschikte combinatie van materialen met een minimum aan oppervlakteslijtage is daarom gewenst. Uit ons experimenteel onderzoek blijkt dat door het beschermen van het zuigeroppervlak met DLC bekleding de wrijving en de slijtage aanzienlijk wordt gereduceerd. Bovendien dient de zuiger/cilinder speling verkleind te worden om het vermogensverlies ten gevolge van gaslekkage te verminderen. Indien echter de zuiger/cilinder combinatie niet goed ontworpen, dan zal dit inherent leiden tot het risico van vastlopen. Een alternatieve ontwerpoplossing is daarom onderzocht, waarbij de zuiger zelf (gas) smerend is. Een lage of zelfs afwezige slijtage en vrijwel geen wrijving zijn hierbij de belangrijkste voordelen die aldus een lange levensduur garanderen. De afwezigheid van oliesmering en een nauwe zuiger/cilinder passing leidt tot hogere eisen die aan het ontwerp gesteld worden. De technicus wordt vaak geconfronteerd met diverse verschijnselen die tijdens bedrijf optreden, bijvoorbeeld tribologische effecten, materiaalvervorming, warmteoverdracht en vloeistofstroming. Een nauwkeurige voorspelling van deze processen is belangrijk om een nieuw ontwikkeld ontwerp te analyseren. Twee numerieke modellen werden toegepast. Voor de analyse van de warmteoverdracht en de vervorming van de zuiger/cilinder samenstelling werd een FEM model gebruikt. Een nieuw voorgesteld zuigerontwerp werd geanalyseerd, dat voordelen geeft ten opzichte van conventionele ontwerpen. Voor de dynamische analyse van een gasgesmeerde ringloze zuiger werd een FDM model ontwikkeld. Beide modellen bleken effectief gereedschap te zijn voor ontwerpverificatie. Deze modellen kunnen als richtlijn dienen voor het realiseren van een optimaal ontwerp. Ter validatie werd een testopstelling ontworpen om de werking 
van een typische zuigercompressor te simuleren. Met deze opstelling kan tevens de haalbaarheid van nieuwe ontwerpaspecten en de prestaties van nieuwe materialen getest en aangetoond worden. 


\section{Contents}

Summary vii

Samenvatting ix

1 Introduction $\quad 1$

1.1 Piston compressors technology . . . . . . . . . . . . . . 2

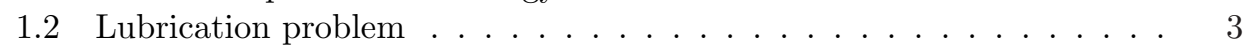

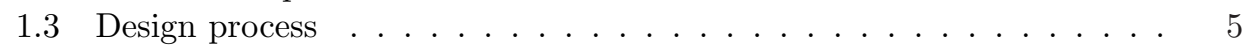

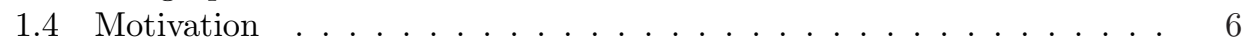

1.5 Aim and scope of the research . . . . . . . . . . . . . . 6

2 Gas spring test rig 9

2.1 General considerations . . . . . . . . . . . . . . . . . . . . . . 9

2.2 Heat transfer . . . . . . . . . . . . . . . . . . . . . . . 10

2.3 Design of the gas spring test rig . . . . . . . . . . . . . . . . . . . . . . . . . .

2.4 Piston design . . . . . . . . . . . . . . . . . . . . . . . . . . . . . . . . . . . . . . . . . .

2.4.1 Conventional design, clearance seal . . . . . . . . . . . . 15

2.4 .2 Alternative designs . . . . . . . . . . . . . . . 16

2.5 Instrumentation . . . . . . . . . . . . . . . . . . . . . . . . . . . . . . . . .

2.5.1 Pressure transducer . . . . . . . . . . . . . . . . 17

2.5.2 Temperature sensors . . . . . . . . . . . . . . 17

2.6 Experiments . . . . . . . . . . . . . . . . . . . . . . . . . . . . .

2.6.1 Gas and surface temperature . . . . . . . . . . . . . 21

2.6.2 Piston temperature . . . . . . . . . . . . . . 21

2.7 Concluding remarks . . . . . . . . . . . . . . . . . 21

3 Coatings tribology 23

3.1 The tribological system . . . . . . . . . . . . . . . . . 23

3.2 Operating conditions . . . . . . . . . . . . . . . . . . . . . . . . . .

3.3 Requirements to the system . . . . . . . . . . . . . . . . . . . . 26

3.4 Candidate surface treatments . . . . . . . . . . . . . . . . . . . 27

3.4.1 Surface hardening . . . . . . . . . . . . . 28 
3.4 .2 Soft coatings . . . . . . . . . . . . . . . . 29

3.4.3 Ceramic coatings . . . . . . . . . . . . . . . . 30

3.4.4 DLC coatings . . . . . . . . . . . . . . . 30

3.5 Properties of DLC coatings . . . . . . . . . . . . . . . . . . . . . . . . . . . . . . 30

3.5.1 Mechanical properties . . . . . . . . . . . . . . 31

3.5.2 Tribological aspects . . . . . . . . . . . . . . . . 32

3.5.3 Physical and mechanical interactions . . . . . . . . . . . . . 32

3.5.4 Environmental effects on the friction . . . . . . . . . . . . 33

3.6 Experimental investigation . . . . . . . . . . . . . . . . . . . . . . . . . . . . . 34

3.6.1 Nano-indentation measurements . . . . . . . . . . . . 35

3.6.2 Friction and wear measurements . . . . . . . . . . . . 37

3.7 Discussion . . . . . . . . . . . . . . . . . . 45

3.8 Conclusions . . . . . . . . . . . . . . . . . . . . 47

4 FEM-ALE model for thermo-mechanical distortion analysis of $\begin{array}{ll}\text { sliding components } & 49\end{array}$

4.1 Types of FEM formulations . . . . . . . . . . . . . . . . 50

4.2 Problem formulation . . . . . . . . . . . . . . . . . . 51

4.3 Weak equilibrium . . . . . . . . . . . . . . . . . . . . . 54

4.4 FEM discretization . . . . . . . . . . . . . . . . . . . . . . . . . . . . . . . . . . . 55

4.4 Interpolation functions . . . . . . . . . . . . 56

4.4.2 Thermal discretization . . . . . . . . . . . . . 56

4.4 .3 Mechanical discretization . . . . . . . . . . . . . 58

4.4 ALE formulation . . . . . . . . . . . . . . . . . . 59

4.4 .5 Modeling contact . . . . . . . . . . . . . . . 60

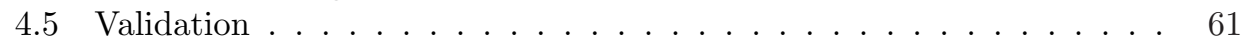

4.6 Concluding remarks . . . . . . . . . . . . . . . 63

5 Analysis of distortions of piston/cylinder assembly 65

5.1 The analyzed system . . . . . . . . . . . . . . . . 65

5.2 Transient analysis . . . . . . . . . . . . . . . . . . . . . 67

5.3 Alternative piston design . . . . . . . . . . . . . . . . 68

5.3 .1 The effect of operating frequency . . . . . . . . . . . 69

5.3 .2 The effect of piston material . . . . . . . . . . . . . 70

5.4 Concluding remarks . . . . . . . . . . . . . . . . . 71

6 Stability analysis of a gas lubricated piston $\quad 73$

6.1 Introduction . . . . . . . . . . . . . . . . . . . 73

6.2 Gas lubrication ... . . . . . . . . . . . . . . . . . . . . . . . . . . . . . . 74

6.3 Literature survey . . . . . . . . . . . . . . . . . . . . . . . . . 76

6.4 Problem formulation . . . . . . . . . . . . . . . . . . . . . . . . . . . . . . 77

6.5 Secondary piston motion model . . . . . . . . . . . . . . . . . . 78

6.5.1 Gas flow model . . . . . . . . . . . . . . . 78

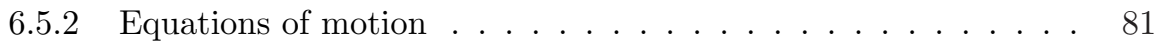

6.6 Numerical procedure . . . . . . . . . . . . . . . . 82 
6.7 Results......................... 85

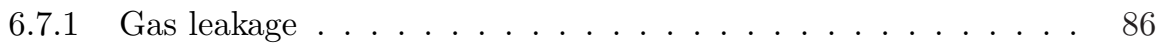

6.7.2 Parametric study . . . . . . . . . . . . . 86

6.8 Concluding remarks . . . . . . . . . . . . . . . . . . . 92

7 Conclusions and recommendations $\quad 93$

$\begin{array}{ll}\text { A Oil-free piston compressors in cryocoolers } & 97\end{array}$

B Closed thermodynamic cycles and refrigeration systems 99

$\begin{array}{ll}\text { C Installations and procedures } & 107\end{array}$

D Coating deposition techniques $\quad 109$

E Environmental effects and superlubricity of DLC coatings 111

F The vacuum tribo-tester $\quad 113$

G Friction measurements performed in vacuum $\quad 115$

$\begin{array}{ll}\text { Nomenclature } & 119\end{array}$

$\begin{array}{ll}\text { Acknowledgments } & 123\end{array}$

$\begin{array}{ll}\text { References } & 125\end{array}$ 



\section{Introduction}

The title of this thesis is: 'On the design of lubricant free piston compressors'. A lubricant can be defined as a substance, which introduced between sliding surfaces, reduces the friction between them, improves efficiency and reduces surface wear. Commonly used lubricants are different types of liquids, mainly oils. Such lubricants however, need to be avoided in this design. The expression 'lubricant free' in the title means no oil-lubricants. Other substances like gases or dry lubricants are acceptable in the analyzed system.

Compressed gases are utilized in various processes including large industrial food installations or medical applications. Specific examples are refrigeration systems including also cryocoolers. In refrigeration systems the piston compressor used to compress and circulate the working gas is a critical component. The compressor performance controls the degree of cooling and efficiency achieved in the system. Conventionally, the compressor's sliding surfaces are oil lubricated to reduce friction and wear. However, in compression of clean and speciality gases as well as in refrigeration systems the presence of an oil lubricant is a common problem. In medical applications or any other where high pureness of the compressed media is required, the oil must be excluded. In the compressors used in cryogenic systems the carry over of an oil to the working fluid must be limited to avoid contamination and hence deterioration of the gas quality and cooling performance. Without the presence of an oil lubricant the compressed medium will remain clean. This, however, necessitates developing advanced surface treatments and coatings, capable of functioning under stringent frictional sliding operating conditions. Alternatively, a different piston lubrication concept can be worked out where frictional sliding is avoided.

In general, piston compressors must fulfill specific requirements due to their inherent use. First of all, lifetime and reliability requirements which are mainly related to material wear and the compressor design. A major challenge in the design is to reduce the potential of material wear in the critical components. The second requirement is an energy efficient gas compression. One of the main contributions 
to energy losses during compression stems from sliding friction. Another factor determining compression efficiency is the piston/cylinder clearance. The clearance must be minimized in order to prevent gas leakage out of the compression space.

\subsection{Piston compressors technology}

During the compression process, the piston should form a sliding seal so that the gas is compressed without any or with acceptable leakage. This means that a very close fit to the cylinder bore is needed. Because of temperature and other engineering and economical reasons this is not practical. Naturally, maximum piston leakage occurs as the piston approaches the end of its stroke because differential pressure across the piston is the highest at this point. This leakage causes both volumetric and power losses. Piston rings are therefore used. Piston rings have many variations, but all follow the same principle of a thin metallic ring, which tends to push out against the cylinder wall and make a tight sliding fit.

a)

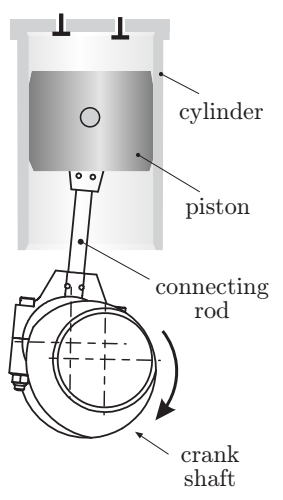

b)

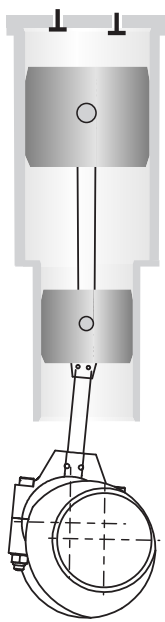

c)

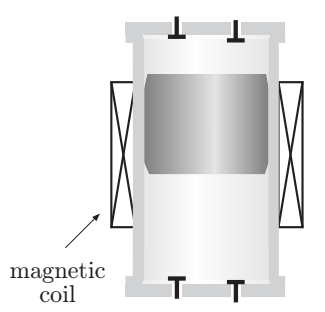

d)

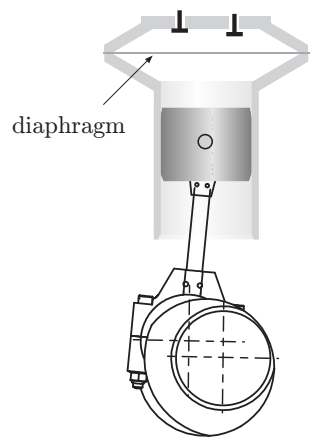

Figure 1.1: Different compressor types, a) crank driven, b) crosshead, c) free moving piston, d) diaphragm

The basic piston reciprocating compressor is a single cylinder with the compression space on one side of the piston, see figure 1.1a. The construction is similar to an automotive engine and uses the piston skirt to guide it in the cylinder. The piston is mechanically attached to a driving mechanism, a crank shaft, via the connecting rod. Its motion is constrained by the motion of the driver, thus stroke and the main position of the piston is predefined. During operation side forces are generated on the sliding surfaces. Generally the bearings, wrist pin and piston rings are 'splash' lubricated by oil. In some other cases, an oil pump is added and the components are pressure lubricated. Some compressors are designed with a separate crosshead to 
guide the piston in the cylinder instead of depending on the piston skirt to provide the guiding, see figure 1.1b. This design has many advantages. First it eliminates contact stresses on the piston skirt. Further, it separates the crankcase from the cylinder allowing control of oil migration to the working piston cylinder assembly. This necessitates a longer piston rod on which a collar or oil deflector is installed. The disadvantage is the limit in rotating speed. In addition, those compressors are heavier and more expensive.

Free piston compressors include those where the piston is supported with either a gas or flexure bearing, see figure 1.1c. With a gas bearing the piston and cylinder surfaces are separated by a thin layer of gas under pressure. The piston is floating on the gas which is the same as the working fluid. Flexure bearings suspend the moving piston by mechanical springs that offer a high radial stiffness and allow easy movement in axial direction. In free piston devices, energy is supplied or removed by an electrical linear alternator. This excludes the need for a linkage and reduces the number of moving parts. Due to the unconstrained piston motion, both the stroke and mean piston position can be modulated during operation. It makes the device more versatile since the piston motion can be adjusted continually to achieve an optimum performance. Another advantage is the lack of side forces since all driving forces act along the line of motion. In some designs friction and wear are nearly eliminated by the use of non-contact gas or flexure bearings. This reduces the high friction losses and wear rates associated with crank devices. Such a solution extends substantially compressor's lifetime and reliability. In practice, the electromagnetic driving forces cannot efficiently generate enough power to drive the piston. To enhance the motion, the piston is mounted using an elastic element so that its natural frequency is close to the intended operating frequency. By driving the system close to resonant frequency less power is needed. However, if the driving frequency or natural frequency changes, the system will move away from resonance and require more input power.

\subsection{Lubrication problem}

A new lubrication concept for piston compressors is needed which excludes oil lubricants. The functional performance of a lubrication system is determined by friction and surface wear. Lubrication failure can be determined by these two parameters. A lubricant is defined as a substance that reduces friction and wear providing smooth movement between sliding surfaces and a satisfactory lifetime for the machine. Most often liquid lubricants are used such as mineral oils, synthetic esters or water. The lubricant may also be in solid state, like teflon, various greases used in rolling bearings, or gases for use in gas bearings. In principle failure will take place when the lubrication no longer fulfills the function for which it was designed. In the case of a piston compressor the sliding components can rub destructively over each other, thereby generating heat or causing vibrations leading to damage and, in the end, to failure.

From experiments it follows that three lubrication regimes can be distinguished. 


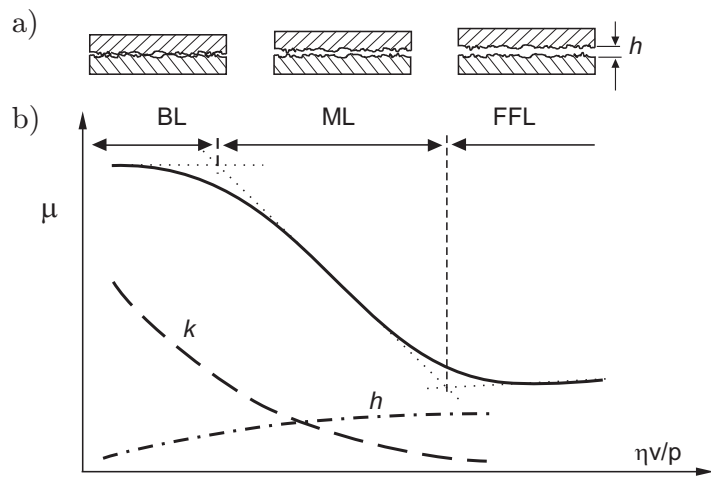

Figure 1.2: a) lubrication regimes and b) Stribeck diagram indicating coefficient of friction $\mu$ as a function of relative velocity. The volumetric wear coefficient $k$ and separation between the sliding surfaces $h$ is also depicted

Each lubrication regime can be associated with its frictional behavior, which can be represented with the Stribeck diagram. An example is given in figure 1.2, the vertical axis represents the coefficient of friction, on the horizontal axis is the dimensionless number associated with the relative surface velocity. Material wear rate $k$ and separation between the sliding surfaces $h$ associated with the different lubrication regimes are also plotted. The lubrication regimes are [9]:

1. Boundary Lubrication (BL): Interaction occurs between the sliding surfaces and the solid surface dominates the contact. Because of surface roughness contact occurs at local spots therefore the load is carried by the real area of contact which is smaller than the apparent contact area. The difference in relative velocity is accommodated in or at the interface of the interacting asperities of the solid surface causing stresses in or at the interface. In a piston compressor with no oil the sliding interface will be stressed by both mechanical and thermal loads. This unavoidably leads to material wear.

2. Full Film Lubrication (FFL): The sliding surfaces are separated and the applied load is entirely carried by the gas under pressure which results in very low friction. In FFL wear is virtually absent. Material failure may occur due to thermal cyclic load. However, this can occur in every lubrication mode.

3. Mixed Lubrication (ML) is the intermediate regime. In this regime friction arises from the combination of a contact at the asperity interfaces and friction caused by shearing the lubricant film in the rest of the contacting region. 


\subsection{Design process}

There are various descriptions of design process available in the literature. Typically a design process follows a logical sequence of events that are common in engineering design. According to van den Kroonenberg there are three most important steps ( figure 1.3) in the design [57].

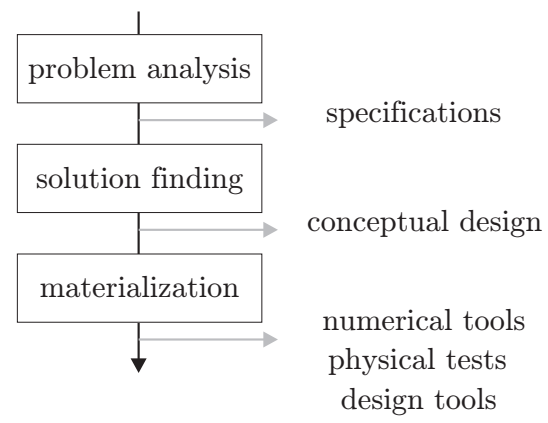

Figure 1.3: The design process

A design process usually begins with a problem analysis. The results of the analysis specify functional requirements of the designed system that has to satisfy a given set of needs. Those needs follow from the designed machine parameters, i.e. capacity, working conditions, materials, maintenance intervals (lifetime). In a piston compressor design different sub-problems can be specified (i.e. lubrication of the crank shaft and connecting rods, etc). This research however will concentrate exclusively on the lubrication problems associated with the ringless piston cylinder assembly. Specifically, working in an environment as in the compression section of Stirling cooling machines. The piston cylinder assembly is mainly responsible for lifetime of those machines.

In the second step, solution finding, different ideas that satisfy the specified requirements are transferred into design concepts. In this work mainly two design concepts will be investigated, based on: minimizing friction with DLC coatings (BL regime) and gas lubrication (FFL regime).

Developing a lubricant free piston compressor for the particular application, the designer will be confronted with many effects, e.g. tribological, material deformation, heat transfer, fluid flow. An accurate prediction of those effects is important to be able to investigate the proposed design. The design concepts in this work will be analyzed with two numerical models. Both models proved to be effective numerical tools for design verification and can provide guidelines to achieve an optimal design. For validation purposes, an experimental test set-up was designed. The feasibility of novel design aspects and the performance of selected materials can also be tested and demonstrated on this set-up. 


\subsection{Motivation}

Construction of an oil free piston compressor is interesting for reasons of cryogenic cooler performance. In addition, it can be used for specific applications where lubricants are not allowed, like the process industry and in medical applications.

In the conventional solution, the sliding surface of the piston compressor is lubricated with an oil to diminish the friction coefficient. However an oil lubricant presents a source of pollution and contaminates the working fluid which results in decreased cooling performance and lifetime. An oil free compressor would require less machine parts, like oil pumps, filters etc. Oil scraping rings are unnecessary and compression rings may be omitted as well. The consequence of this will be that the parts need to be produced in such a way that friction, thermal expansion and thermal fatigue do not cause problems on the piston/cylinder interface.

Live tests performed on various Stirling coolers have shown, that using teflon-based coatings, the life time is limited to about 4,000 to 10,000 hours mean time to failure (MTTF), depending on the cooler type becouse of surface wear in the piston cylinder assembly. In order to ensure MTTF values of more than 20,000 hours, the wear has to be reduced.

The development of high performance long lifetime and maintenance free piston compressors is very important as, in the next few years, the cryocoolers market is expected to expand substantially. This is caused by fast growing application of localized gas liquefaction, gas storage and transportation, electronic and thermal noise suppression by cryogenic cooling and the High Temperature Superconductivity (HTS) technology.

\subsection{Aim and scope of the research}

The aim of this research is to develop novel design of high performance piston compressors for the application to refrigeration systems excluding oil lubricant. The lifetime and reliability are identified as the most important aspects which are mainly attributed to the piston surface wear. Moreover, considering efficient gas compression, the energy losses due to frictional sliding must be low. Another factor determining compression efficiency is the piston/cylinder clearance. The clearance must be minimized in order to prevent gas leakage out of the compression space. With the view to advanced materials and with the help of numerical modeling, various design concepts are explored.

In Chapter 2 the proposed design and solutions will be validated and demonstrated on the most elementary set-up which is the gas spring compressor test rig. The gas flow and heat flux in the materials predicted with numerical simulations are experimentally validated in the test rig. With the obtained results design guidelines will be established for oil free piston compressors with a minimum piston/cylinder 
clearance. The thermal phenomena occurring in a piston compressor are investigated in a parallel running PhD project performed in the Group of Thermal Engineering.

In section 1.2, it was indicated in what lubrication regimes a piston compressor can operate and what the consequences are regarding friction and material wear. In this thesis two distinct approaches to solve the piston lubrication problem will be discussed.

In Chapter 3 we concentrate on BL regime and therefore tribology of preselected materials is investigated in inert atmosphere. Considering the tribological system it is concluded that chemical aspects play an important role. Therefore the chemistry of the compressed medium is of concern. With a view to the advanced materials, design and tribology issues, several potentially applicable materials and surface coatings are considered and tested. Wear rate of the materials has to be reduced to an acceptable level. In tribological systems very often surface properties are modified since the surface determines friction and wear between sliding bodies. Therefore coatings with a low coefficient of friction are studied. Tribological tests under conditions as expected in the compression section of a Stirling cooler were performed.

An important aspect is the control of mechanical tolerances at a large temperature range. For high performance of the piston compressor a narrow tolerance between piston and cylinder must be realized. For that reason a numerical model based on finite element method (FEM) is used to provide a better understanding of the matched materials and their response under given operating conditions. The FEM model will be used to predict deviations in size of the piston and cylinder caused by thermal expansion. This is discussed in the Chapter 4. Results of thermo-mechanical distortion analysis are presented in Chapter 5 .

In the second approach we consider the piston operating in the FFL regime. The possibility of designing a gas-lubricated piston is studied in Chapter 6. Low viscosity of the gas offers virtually frictionless and wearless operation. Consequently such design ensure the highest lifetime. In order to fully benefit from the advantages offered by full film lubrication (FFL) the piston must be properly designed. Many design parameters are involved. To optimize the design a numerical model has been developed based on finite difference method (FDM). The model takes into account the piston geometry, dynamic properties and operating conditions. The influence of various design parameters and operating conditions on the, so called, secondary piston motion (SPM) has been analyzed.

\section{Appendices}

- Appendix A, presents some requirements to piston compressors in cryocoolers

- Appendix B, briefly outlines the basic thermodynamic cycles used in refrigeration systems and selected devices based on Stirling and Rankine cycle. 



\section{Gas spring test rig}

This chapter deals with the gas spring test rig developed within this project at the University of Twente. The test rig is designed to simulate operating conditions of a typical piston compressor. The feasibility of novel design aspects and the performance of selected materials can be tested and demonstrated on this set-up.

The power losses during compression are mainly attributed to the gas leakage out of the compression space. Therefore, control of the piston/cylinder clearance is essential. The material thermal expansion is mainly responsible for components deformation and change of the piston/cylinder clearance during operation. Heat transfer phenomena occurring in gas compression space need to be accurately predicted since the amount of heat transferred to the surrounding solid surfaces, e.g. piston and cylinder liner determines the material's temperature. The thermal phenomena occurring in a piston compressor will be explored with this set-up.

The potential of various piston designs to reduce gas leakage is discussed. Further, the gas spring test rig construction is outlined. Same experimental results obtained within this project will be discussed at the end.

\subsection{General considerations}

Material wear and oil deterioration are the main factors which limit lifetime of refrigeration systems. An oil-free, ringless piston compressor has the potential to eliminate these problems. The gas spring is basically the most elementary piston compressor where a working gas closed in a compression space is compressed/decompressed by means of a reciprocating piston. A cyclic pressure variation is generated. Associated with these pressure variations are flows and temperature variations. As can be proved analytically and experimentally the dominant factor affecting the compression performance is the piston/liner clearance. Because of the clearance the gas will escape partially from the compression space. 
This reduces the pressure variation and consequently the gas temperature. Gas flow within the narrow clearance can be considered as laminar and it is proportional to [9] [90]:

$$
Q \doteqdot \frac{c^{3} \Delta p^{2}}{\eta L}
$$

where the radial clearance $c$ is dominant in the equation. $L$ is the seal length and the pressure difference along the piston is denoted by $\Delta p$. Figure 2.1 shows results of the pressure drop analysis for different gases in a piston/cylinder clearance as a function of time. The calculations were done for the following geometry: piston length $\mathrm{L}=20$ $\mathrm{mm}$, diameter $\mathrm{D}=50 \mathrm{~mm}, \mathrm{c}=0.03 \mathrm{~mm}$ and $\Delta \mathrm{p}=200 \mathrm{kPa}$.

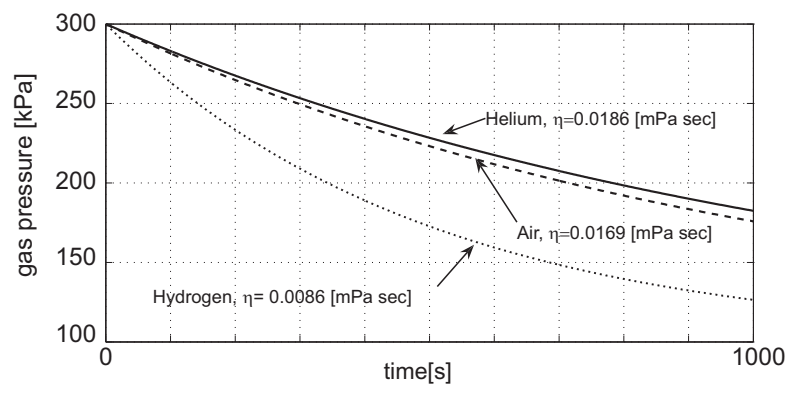

Figure 2.1: Gas pressure drop in a clearance seal

\section{$2.2 \quad$ Heat transfer}

The in-cylinder heat transfer is a complex combination of mainly two processes: conduction and forced convection schematically shown in figure 2.2. In the compression space the surfaces are exposed to a transient convective heat flux. The gas flow induced by the piston motion has a transient boundary layer. In the boundary layer the gas velocity rises from zero (at the wall) to the bulk value. Due to the gas velocity the temperature rises (and decreases) from the wall temperature to the temperature of the bulk gas and a thermal boundary layer is formed, see figure 2.2 . The characteristics of the boundary layer like thickness and turbulence determine the gas/solid heat transfer. Two other major parameters of heat transfer are the gas density and the gas temperature. Hence there will be a periodically oscillating heat flux between cylinder wall and gas. The temperature of the solid cylinder material will then be periodically fluctuating as well. However, the piston speed is high and the temperature fluctuations only penetrate a small distance into the cylinder wall. Because of the complex connection between fluid motion, pressure and temperature, accurate modeling of heat transfer is conducted by combining thermodynamics with Computational Fluid Dynamics (CFD). Those activities are carried out within the parallel running PhD project in the Group of Thermal Engineering. The main interest of the investigation lies in prediction of the instantaneous heat flux [59] [60]. 

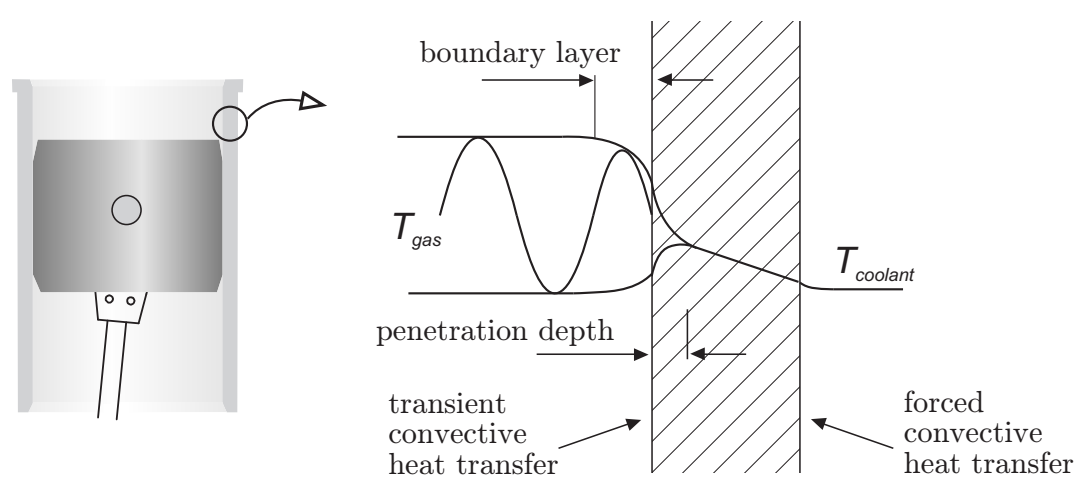

Figure 2.2: Heat transfer in a cylinder liner wall

\subsection{Design of the gas spring test rig}

The gas spring test rig is designed and built to accommodate an oil-free, ringless piston operation. Construction of the apparatus allows easy adaptation in order to investigate a range of operating conditions. Therefore the apparatus has a modular construction. The characteristic operating conditions are given in table 2.1. Since the gas spring will be used to explore the novel design under various parameters the components can be exchanged with the newly designed ones. The minimum clearance is one of the issues to be investigated. Experimenting with a tight piston/liner fit means that the risk of seizure is inherent thus special safety solutions are provided.

The apparatus has a crosshead type construction and is constructed on the base of the Stirling cryogenerator type SPC-1 (Stirling B.V./Netherlands), see Appendix B. The lubricated piston of the compressor base is used as a crosshead, driving the 50 $\mathrm{mm}$ diameter unlubricated piston of the gas spring, see figure 2.3. This arrangement reduces side forces on the piston sliding surface and also separates the working gas from oil lubricant contained in the crank case. The base compressor originally is equipped with an oil lubrication system. The lubricant is pumped through oil cooler, filter and further the main bearings are lubricated. Via small channels in the crank shaft and the connecting rods the oil is supplied to the gudgeon pin.

\begin{tabular}{lll}
\hline piston stroke, & 0.052 & {$[\mathrm{~m}]$} \\
piston diameter, & 0.05 & {$[\mathrm{~m}]$} \\
operating speed, & $<3000$ & {$[\mathrm{RPM}]$} \\
& $<50$ & {$[\mathrm{~Hz}]$} \\
compression ratio, & $2 . .8$ & {$[-]$} \\
working gas & helium & \\
\hline
\end{tabular}

Table 2.1: Characteristic operating parameters of the gas spring test rig 


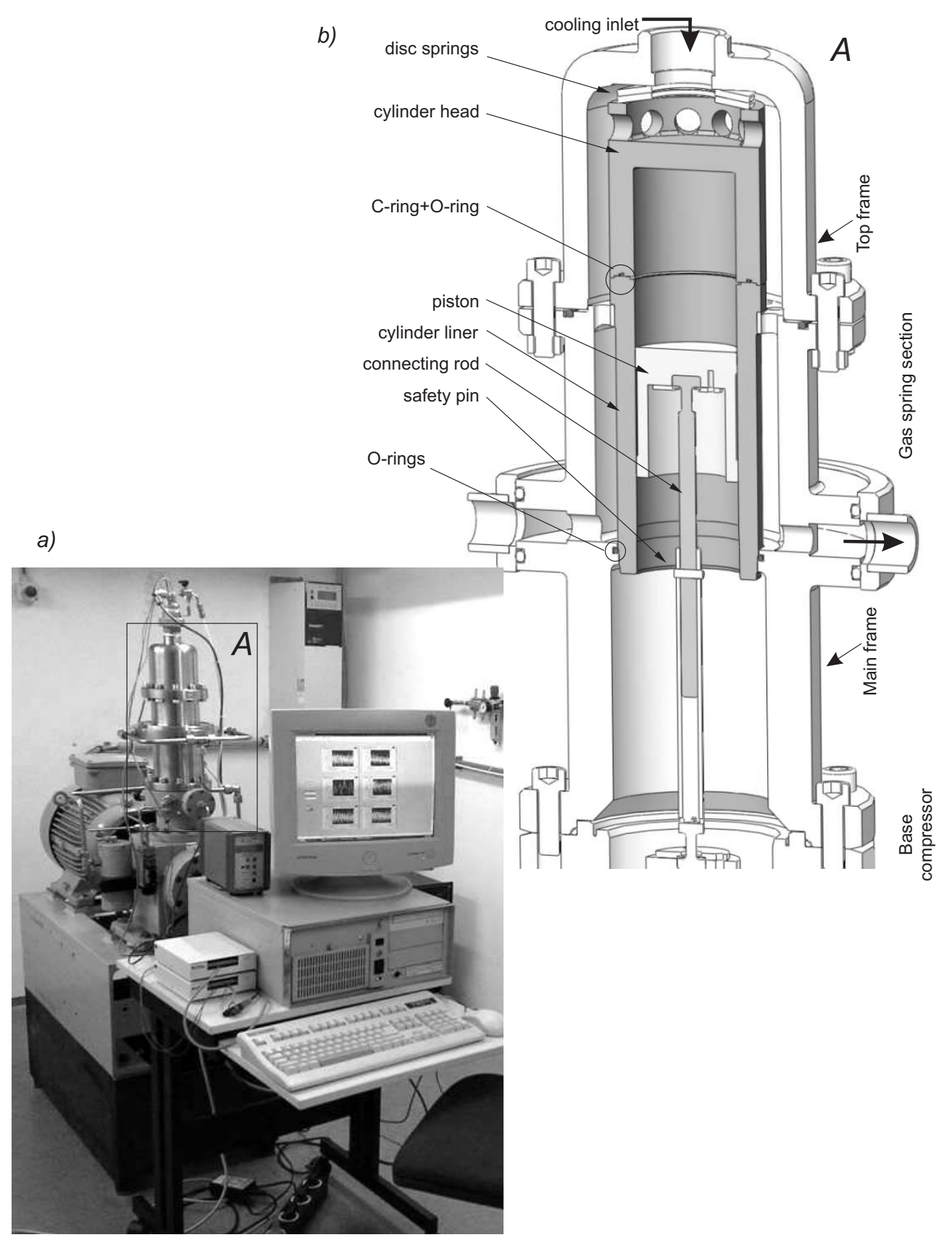

Figure 2.3: Gas spring test rig a) and cross view of the gas spring section b) 


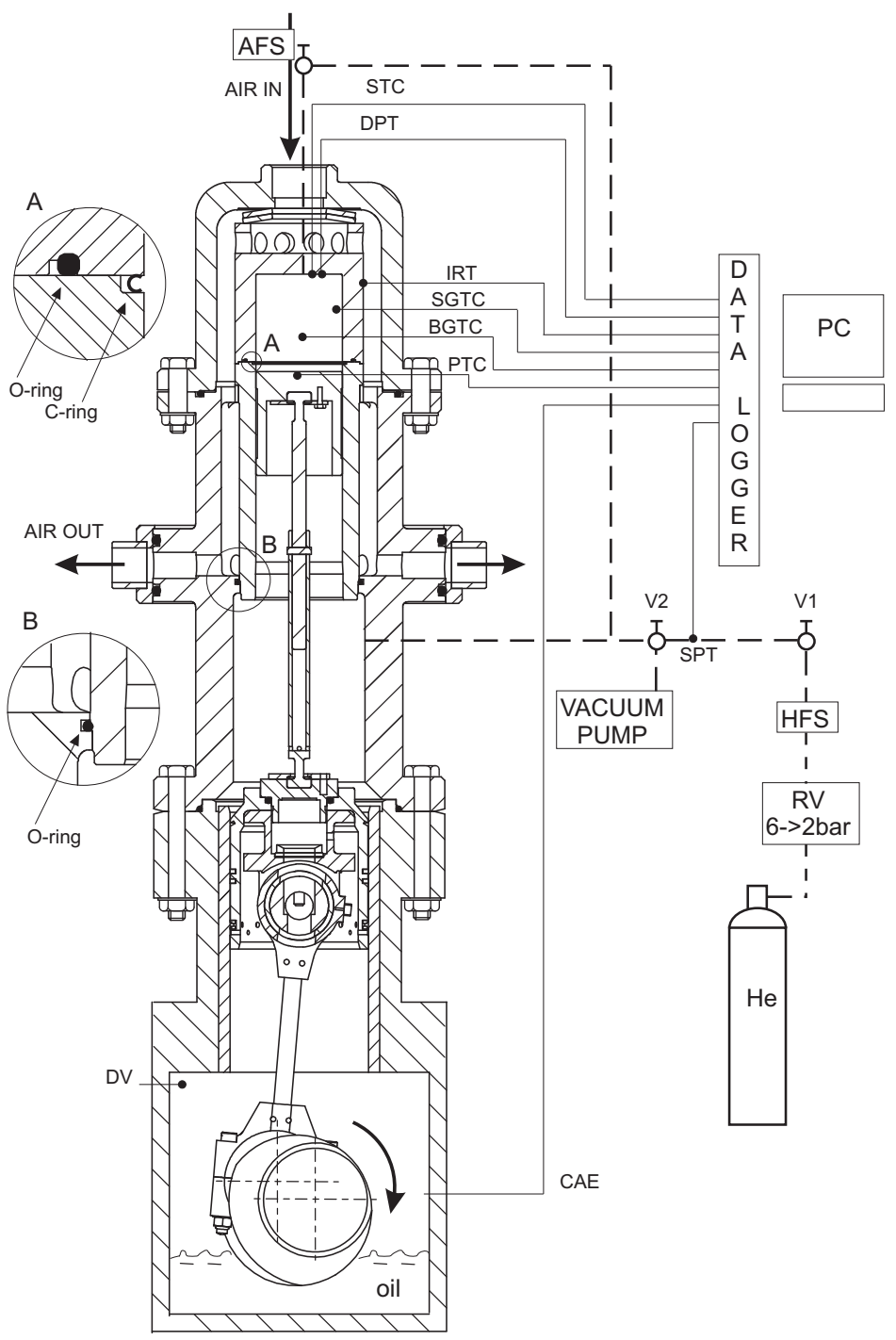

Figure 2.4: Cross section of the gas spring test rig and the installation set-up scheme, with the following abbreviations: AFS air filter system, STC surface thermocouple, DPT dynamic pressure transducer, IRT infrared temperature sensor, SGTC surface gas thermocouple, BGTC bulk gas thermocouple, PTC piston thermocouples, SPT static pressure transducer, HFS helium filter system, RV pressure reduction valve, V1 one-way valve, V2 two-way valve, DV discharge valve, CAE crank angle encoder. Sensor connections are depicted by continuous lines and the dashed lines depict the helium installation, the thick arrows show flow direction of the cooling air. 
a)

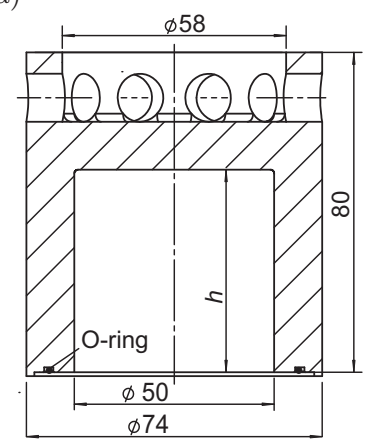

b)

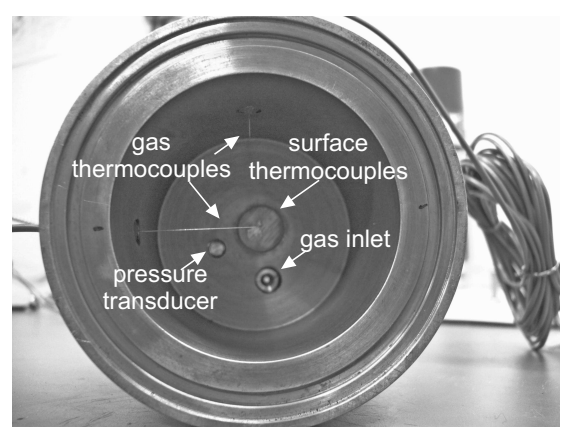

Figure 2.5: The cylinder head, a) cross-section drawing and b) view on the installed sensors

The gas spring section consists of the piston, cylinder liner and cylinder head assembled in the outer casing. This consists of two parts: namely the main frame and the top frame. The frames provide a support structure for the apparatus therefore need to be rigid and manufactured with high accuracy. Special attention was paid to the concentricity of the main frame with respect to the base compressor since after assembly any misalignment would cause side forces on the piston/liner interface. The gas spring piston and the base compressor piston are rigidly connected by a telescopic connecting rod joined with a safety pin. The safety pin is designed to break if too high loads occur. In case of seizure or any other excessive forces the pin will disconnect the two pistons preventing the base compressor and the gas spring section from damage, see figure 2.3. The connecting rod contains a reduced cross section on its ends to act as an elastic hinge in order to compensate eventual piston side forces. The cylinder liner is inserted after the piston and the main frame have been assembled. The liner is resting on the inner flange of the main frame. The lower part of the liner is resting on the O-ring sealing the cooling air from the buffer space containing the working gas. The cylinder head/liner interface is dual sealed to minimize the gas escape from the compression space to the cooling channel. A standard O-ring is placed in the half thickness of the interface. On the cylinder edge the special high precision, gas tight C-ring (HTMS/ Belgium) is mounted. More details can be seen in figure 2.4. A high sealing level is provided due to high contact forces. The sealing concept is based on elasto-plastic deformation of the seal during compression when assembled, in addition the internal gas pressure creates extra sealing load. The seal is made from the nickel alloy X-750. The cylinder head is pressed down to the liner with two disc springs. The top frame is bolted to the main frame loading the disc springs. The cylinder head is made from copper alloy of high thermal conductivity. With high thermal conductivity the thermal penetration depth is expected to be larger. All parts of the gas spring are exchangeable. By adapting the dimension, $h$, see figure 2.5a, experiments with different compression ratios can be carried out. Most of the sensors are installed here, see the photograph in figure $2.5 \mathrm{~b}$. In the cylinder head wall the transient heat flux on 
the surface will be measured by fast response thermocouples. Gas temperature and pressure characteristics will be recorded as well.

The apparatus is driven by an AC electric motor, power $11 \mathrm{~kW}$ (Loher/ Netherlands) type ANGA 160M stabilized in speed by a flywheel. A frequency controller (Dynavert/ Netherlands) is coupled with the electric engine. This allows to set the rotating speed in the range of 1 up to 3000 RPM. The electric motor and compressor shaft are connected with a transition piece, Centaflex X-series (Stemin/ Netherlands) which is relatively stiff in the torsional direction and can adjust elastically to compensate possible misalignments between the electric motor and gas spring shaft. The instantaneous crank shaft position is recorded by a crank angle encoder (Leine\&Linde/ Sweden), type RHI 503. The sensor is mounted on the free end of the electric motor. The sensor has a resolution of 3600 pulses per revolution.

\subsection{Piston design}

A tight clearance must be maintained between the piston and cylinder liner from cold start to rated power conditions. Ideally, an intelligent piston is desired which can control its diametric thermal expansion to be consistent with the liner. Several piston designs have been created to address this problem. Unfortunately, most of these are either too expensive or have unsatisfactory performance.

\subsubsection{Conventional design, clearance seal}

One design solution to control the piston/liner clearance can be maintained with a careful material selection. The minimum clearance is mainly determined by the two factors: thermal expansion of both piston and liner and the practical finite clearance obtainable due to the machining constraints. Manufacturing the piston and liner from materials of the same coefficient of thermal expansion does not solve the problem of changing clearance due to the following reasons: 1) The average piston temperature differs from that of the liner; the liner outer surface is usually cooled unlike the piston. 2) Piston and liner have a temperature distribution along their lengths. 3) The temperature gradients and averaged temperatures difference between piston and liner vary for each load condition and during transients. To prevent piston seizure the clearance must account for the maximum temperature differential between the hottest part of the piston and the coolest part of the liner at any operating condition. This minimum design clearance can be reduced by manufacturing the components from materials of a low and similar coefficient of thermal expansion. In order to reduce the temperature gradients within the components, a material of high thermal conductivity is desired. 


\subsubsection{Alternative designs}

A candidate ringless piston design concept is shown in figure $2.6 \mathrm{~b}$. The piston consists of two main parts; the crown and rod are one part, the skirt is the second. The two parts are in contact along a seating surface and are held there by a spring. During running the piston crown operates at higher temperature than the skirt. The skirt temperature is held low by ceramic inserts that reduce the heat flux to the skirt. By this means, the skirt temperature is made to be approximately the same as the liner wall; this is because the skirt is isolated from heat flux from the crown and is in close proximity to the liner wall. This encourages heat flow between wall and skirt by convection and radiation and tends to make their temperatures equal. The crown thermally expands, but its expansion does not change the minimum clearance between piston and wall, since the minimum clearance is between skirt and wall. When the crown expands radially, it slides on the contact surface with the skirt and compresses the spring, but the clearance is unchanged. Therefore, the clearance change is a function only of the temperature of the skirt and the liner. Since these temperatures are always approximately equal, the clearance is always approximately constant if the thermal expansion coefficients of the materials are the same. The following drawback of this concept is that the skirt may become unseated at the crown interface under high operating speed due to inertia loads. For compressors with large pistons the concept is feasible; however, for smaller pistons the assembly can be more problematic. Further, a small amount of gas leak is tolerated in this concept. The

a)

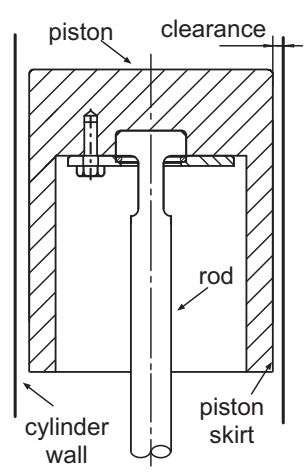

b)

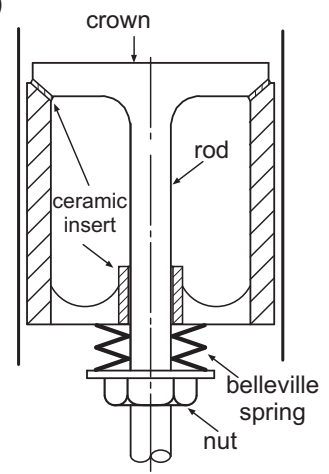

c)

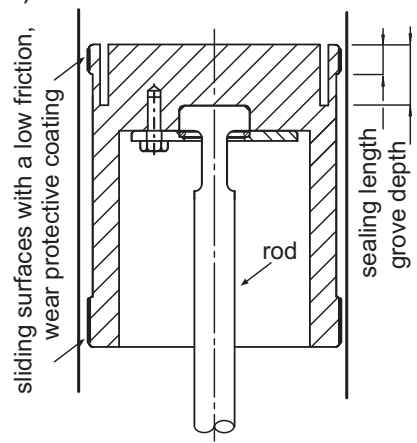

Figure 2.6: Candidate ringless piston designs for an oil-free piston compressor a) clearance seal, b) the T-piston [3] and c) the U-piston

second alternative design having the potential to suppress the gas leak is depicted in figure 2.6c. The piston is constructed similar to the traditionally used elastic lip seals in hydraulic systems. The sealing element could be designed as an integrated part of the piston. The circumferential slot allows the piston skirt to adjust elastically to the liner surface. The rising pressure in the slot during compression and thermal expansion would give rise to the sealing effect. The main advantage is that the gas leakage is almost totally suppressed. Since the sliding surface will be continuously in 
sliding contact it must be covered with a wear protective coating. The application of DLC coating may reduce the coefficient of friction down to 0.02 . Sliding properties of DLC coatings in an oil-free environment will be discussed in the following chapter.

\subsection{Instrumentation}

In order to quantify loss mechanisms associated with the compression and expansion process, the $\mathrm{p}-\mathrm{V}$ work done by the piston on the gas needs to be accurately measured. This requires the gas pressure to be recorded in the function of the gas volume. The gas volume is calculated based on the instantaneous crank position. A typical operating frequency of $25 \mathrm{~Hz}$ means that a sensors response time of less then 0.004 sec is required on the assumption that 100 measured data points for one cycle are sufficient. Heat transfer and flow processes are believed to contribute mainly to the loss mechanisms in cryocoolers [2]. The gas leakage in the piston/cylinder clearance can be determined based on gas pressure measurements in the compression space and in the buffer space. Measurements of transient convective heat transfer on the gas/surface interface requires fast surface and gas temperature sensors. The gas spring test rig is instrumented with the following sensors:

\subsubsection{Pressure transducer}

The gas pressure fluctuations in the compression space are measured with a piezoelectric dynamic pressure transducer (Kistler/ Switzerland) type 6052B1. The sensing element measures the relative stress and is thus unable to record the absolute pressure in the compressed volume. Instead, only the amplitude of the change is measured. Together with the buffer-space absolute pressure measurement, the compressed gas pressure is leveled. With this sensor a high pressure range can be measured: 0-250 bar, high operating temperature up to $400{ }^{\circ} \mathrm{C}$. The signal is amplified with a charge amplifier, type 5011B10Y50 with a very wide frequency range 0 up to $200 \mathrm{kHz}$. The amplifier has a drift compensation module integrated, allowing the piezoelectric transducer to be used as a static transducer as well.

\subsubsection{Temperature sensors}

Eroding-type surface thermocouples are custommade by Nanmac Corporation/ USA. The main body of the probe is made of the same material as the wall (Copper EN 13601, DIN 40500, R250), thus eliminating the errors caused by the differences in material thermal properties. Several reports [34] [35] witness the superiority of these thermocouples over other available constructions for very fast and accurate surface temperature measurements. Instantaneous surface heat flux can also be measured because of one extra reference thermocouple. The back thermocouple is a standard E-type ( 2 wires and a junction) thermocouple, with the tip installed $0.5 \mathrm{~mm}$ from the 


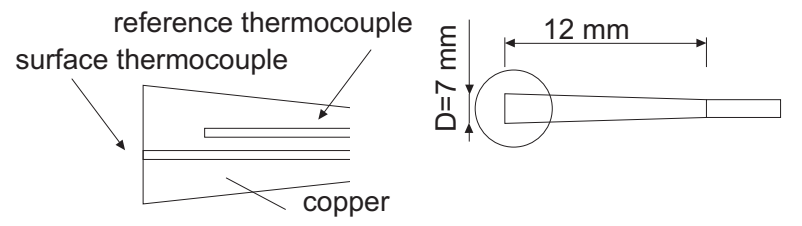

Figure 2.7: Schematic representation of the surface thermocouples

surface. In this way, with the known distance, heat flux will be instantly measured through the temperature gradient. A ribbon of chromel and a ribbon of constantan (Etype), each $0.025 \mathrm{~mm}$ thick, are embedded in the copper probe body and separated by a sheet of mica $0.005 \mathrm{~mm}$ thick. The thermocouple junctions are formed by sanding the front surface of the probe, which creates many microscopic thermocouple junctions.

The instantaneous bulk gas temperature is measured with fast response thermocouples (Paul Beckman/ US) type 03. Those probes have extremely low mass. The junction is $0.2 \mathrm{~mm}$ in diameter together with insulation, which gives millisecond range response. The thermocouple is constructed as a standard E type (chromel-constantan).

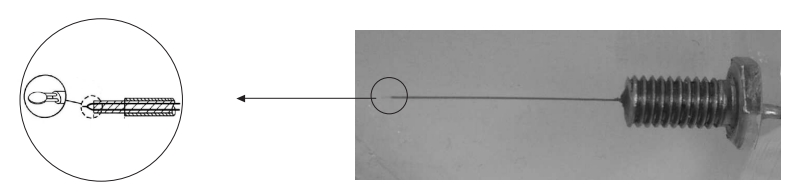

Figure 2.8: Gas thermocouple

One probe $25 \mathrm{~mm}$ in length protrudes in the compression space and measures the bulk temperature of the gas, see figure 2.8. One probe $6.25 \mathrm{~mm}$ in length is mounted in the head wall, ending flush with the surface and measures the surface temperature. A thermally conductive glue is used to fill the little gap between the thermocouple sensing probe body and the surrounding wall. Boron nitride coating (Saint-Gobain) is used due to its high thermal conductivity $(125-300 \mathrm{~W} / \mathrm{mK})$ and low dielectric constant. An infrared sensor (Raytek Corporation), model type MI 20 is used to record the temperature on the outer surface of the cylinder head. The sensor is installed in the top frame.

More details concerning the gas spring test rig instrumentation and procedures are provided in Appendix C.

\subsection{Experiments}

A baseline performance is measured using a bronze piston. For the first measurements we decided to use a typical material. In case of failure, because of the lower piston 
hardness the liner surface would not damage. Bronze is often used for sliding machine elements because of a low friction coefficient ( 0.3 bronze/steel in air). The thermal expansion coefficient of bronze is close to that of stainless steel and the high thermal conductivity will reduce the temperature gradient along the piston. A labyrinth seal can effectively reduce gas leakage in a clearance seal. In the report [17] analytical results were shown indicating that three grooves can reduce gas leak by approximately $30 \%$. A further increase the number of groves would reduce gas leak by about $1 \%$ only. No information was given about the groove dimensions. It has been decided to make three triangular grooves on the sliding surfaces, simply due to the fact that triangular grooves could easily be made. Figure 2.9 shows the design.

$\mathrm{B}$

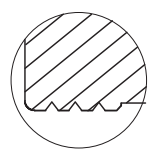

SECTION A-A

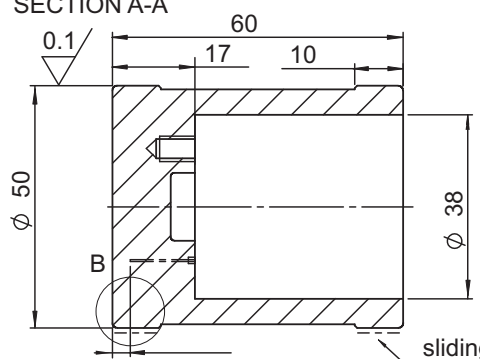

$\left({ }^{*}\right)$ thermocouples depth

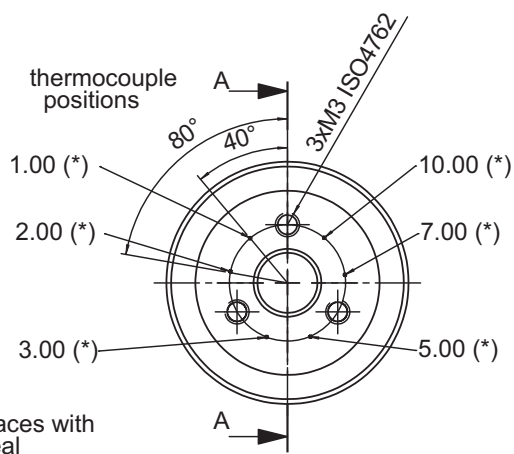

abyrinth seal

Figure 2.9: Piston

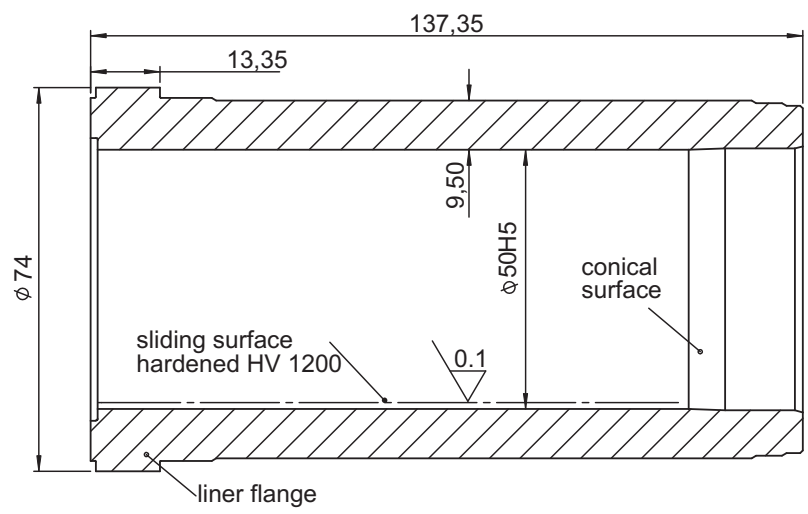

Figure 2.10: Cylinder liner

The cylinder liner is made from austenitic stainless steel (AISI 316L), figure 2.10 shows the drawings. The three main parts can be distinguished: the conical section, the sliding surface and the flange. The conical section is made to allow easier handling during assembly. The sliding surface is hardened according to the Kolsterization 
process. More information about the process is given in Chapter 3. The liner is manufactured with tight tolerances and high precisions, the surface roughness is low, $\mathrm{Ra}=0.1 \mu \mathrm{m}$. The piston presented in figure 2.9 has been mounted, and together

a)

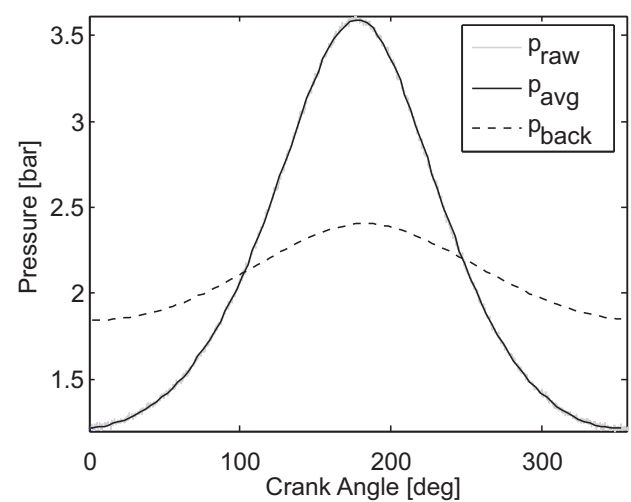

c)

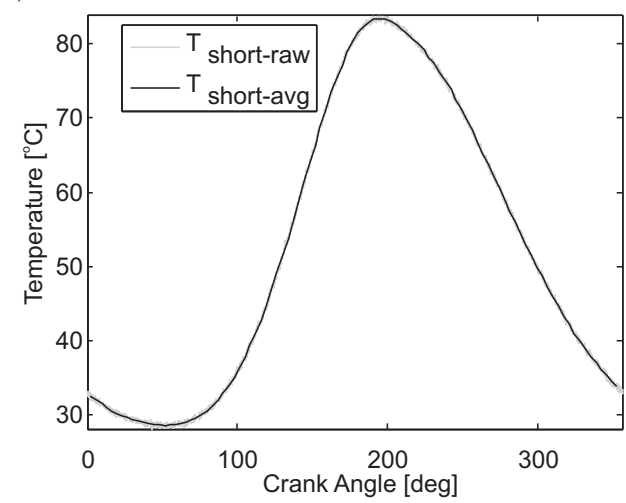

b)

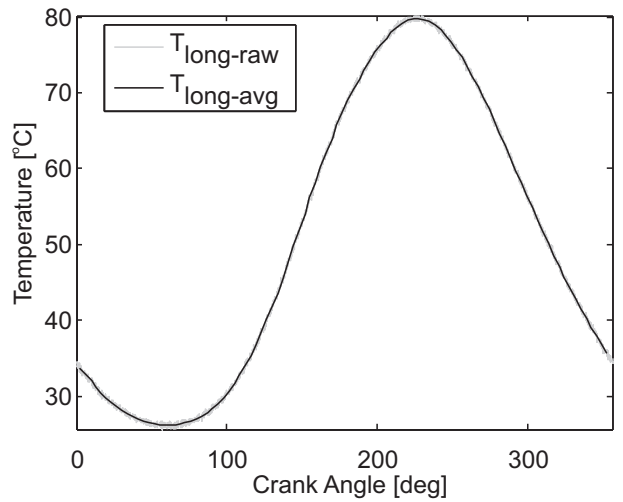

d)

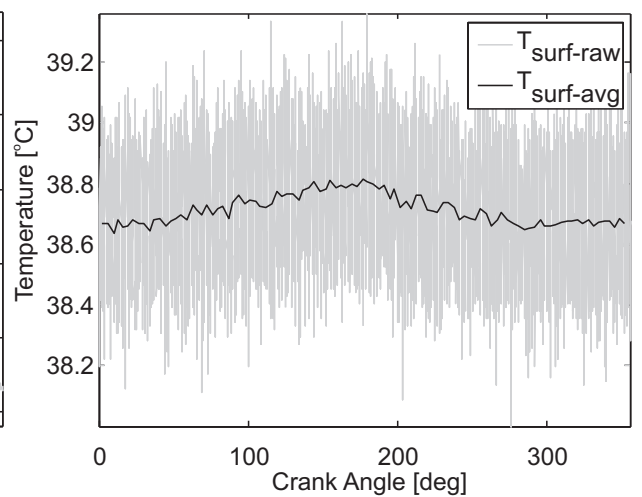

Figure 2.11: Some measurement results, a) Compressed Gas Pressure, b) Compressed Gas Temperature in the bulk, c) Compressed Gas Temperature close to the wall, d) Inner Surface Cylinder Temperature

with a cylinder (measured $\mathrm{D}=50.205$ ), couples to a diameter-clearance of $25 \mu \mathrm{m}$. Experiments were prepared according to the procedure explained in Appendix C. The set-up was vacuumized and charged with helium to a higher pressure (6 bar) and bled several consecutive times to ensure the operating gas purity. For all the experiments helium was finally charged to the pressure $1.5-2 \mathrm{bar}$, left for a long enough time (30-60 $\mathrm{min})$ for all the pressures in the set-up to equalize and come to the thermal equilibrium, and then the set-up was operated. A range of operating frequencies was investigated: $0.5,2,5$ and $10 \mathrm{~Hz}$. Typical measurement results for the $10 \mathrm{~Hz}$ operating frequency are shown in figure 2.11. More measurement data can be found in the work published by Lekic [59] [60]. 


\subsubsection{Gas and surface temperature}

Every measurement was started after a certain steady state was reached. Raw recorded data is span-averaged over the batches of 100 samples in order to clean the noisy signals (raw and avg lines on the graphs). The raw signal displayed on 2.11 has an uncertainty of $0.6 \mathrm{~K}$. With the averaging procedure this amplitude is reduced by a factor of 20 , to a value of $0.03 \mathrm{~K}$. Data is post-processed for the span-averaging, calibration correction of the thermocouples, and the gas pressure leveling.

\subsubsection{Piston temperature}

The pistons have been instrumented with thermcouples to measure the material temperature. The thermocouples (ThermoElectric/ Netherlands) are mounted on various depths in the piston crown, measuring temperature on radially distributed points and 1-10 mm distance away from the compressing surface. They are glued by the thermally conductive silver glue. The thermocouple wires are fixed to the exit ports in the gas-spring frame wall and flexing of the lead wires is unavoidable because of the reciprocating piston. With this, the danger of distorting the signals occur. Performed experimental investigation showed no influence on the measured signal.

\subsection{Concluding remarks}

The design and construction of the gas spring test rig developed during this project was discussed. The test rig is constructed on the base of the SPC- 1 cryogenerator in a crosshead arrangement and it is driven by an electric motor. A frequency controller and the modular construction allows to set up the desired piston operating conditions. The construction of the test rig allows easy access to the gas spring components. Thus, they can be easily exchanged or disassembled for inspection. Working with a tight piston/cylinder fit the risk of seizure is vital. Special safety design solutions are included to prevent the test rig from damage in case of seizure.

For the first experiments materials for the working components (piston and cylinder) were selected based on available characteristic data and the basic tribological tests conducted within this project, see section 3.6.2. In the gas spring the performance of the selected materials and the novel piston design can be tested under conditions simulating piston compressor. The key response is the material wear and the gas leakage in the piston/cylinder clearance.

The first preliminary results and operation of the set-up were demonstrated. At specific locations in the gas spring experimental data, mainly pressure and temperature can be measured. With these, data accuracy of the employed numerical models can be validated and eventual improvements can be made for more accurate prediction. 


\section{Appendices}

- Appendix C, gas spring test rig instrumentation and procedures 


\section{Coatings tribology}

In this chapter we focus on the tribology aspects of dry sliding surfaces in the compression section of a Stirling cooler. The working environment is characterized by specific conditions, namely helium gas, low contact pressure, reciprocating motion. Since liquid lubricants need to be excluded from the compressor a solid lubrication is considered here as the alternative. The aim of this study is a materials selection for both minimum material wear and low coefficient of friction $(\mathrm{CoF})$. With less material wear the maintenance intervals can be extended. Low friction between the sliding components will result in less energy loss and also less heat generated on the sliding surfaces. The resulting lower temperature would also involve less thermal distortions in the components.

The tribological system with the operating conditions are discussed. The commonly used materials and their alternatives are presented. Special attention is put on the diamond-like carbon (DLC) coatings. The combination of wear resistance and very low $\mathrm{CoF}$ in one material makes DLC coatings an ideal choice for the design of sliding mechanical components. Finally, the experimental results obtained from sliding tests performed within this project will be presented.

\subsection{The tribological system}

Considering friction and wear, mechanical, physical as well as chemical aspects play a role. To study tribology in a systematic way, Czichos [14] has developed a so-called system approach. A tribological system is defined as: an entity whose functional behavior is connected with interacting surfaces in relative motion. Friction, lubrication and wear are considered as system dependent characteristics and therefore to study friction, wear and lubrication one needs to look at the total tribological system.

According to Czichos, it is necessary to describe both the function and the structure of the tribological system. A tribological system generally consists of four elements: 
two sliding bodies, a lubricant and the environment. The two sliding bodies have both volume and surface properties. Volume properties are geometry, chemical composition, mechanical properties (e.g. hardness, elastic modulus) and thermal properties (e.g. thermal conductivity). Surface properties are characterized by the surface composition (boundary films) and microgeometry. The environment is characterized by properties like chemical composition, pressure and temperature. The lubricant is the medium between the sliding surfaces, that can be in a fluid or solid state.

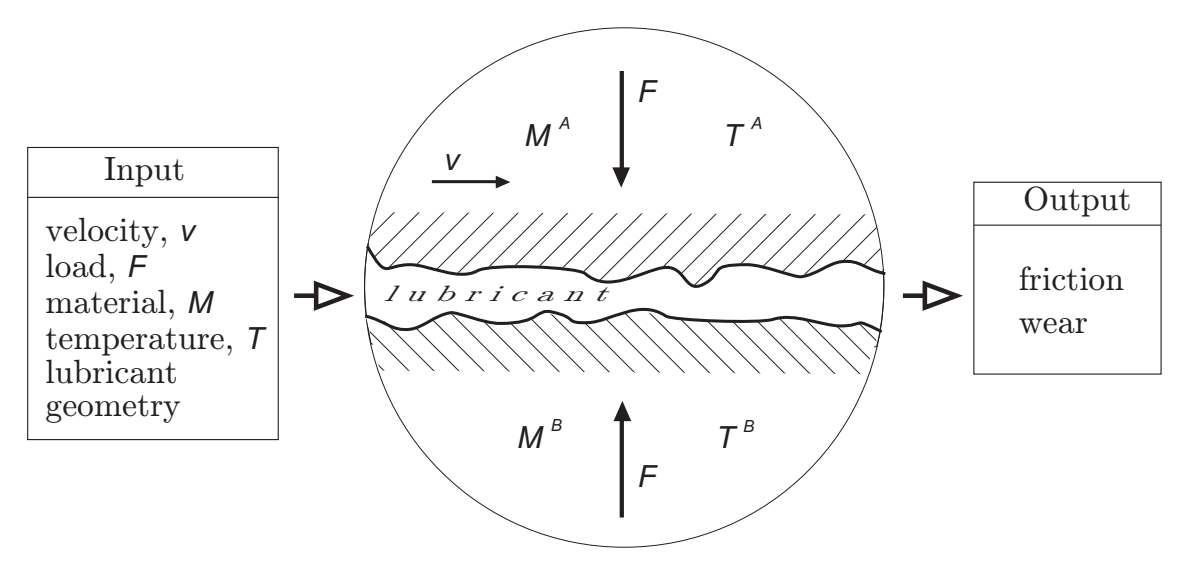

Figure 3.1: The tribological system.

Figure 3.1 shows schematically a tribological system of two sliding bodies made of material $A$ and $B$. The system behavior is determined by the operational parameters: contact load $F$, relative and absolute surface velocity $v$, temperature $T$ and type of motion (e.g. one-directional or reciprocating sliding). The coefficient of friction $\mu$ and the volumetric wear coefficient $k$ are parameters commonly used to quantify the performance of the tribological system.

$$
\mu=\frac{F_{t}}{F_{n}}
$$

where $F_{t}$ and $F_{n}$ are respectively the tangential force and normal load.

$$
k=\frac{V}{F_{n} d}
$$

where $V$ is the volume of material worn off and $d$ is the sliding distance. Typical $\mu$ and $k$ values of common materials measured in an inert atmosphere, sliding on steel are given in table 3.1 . 


\begin{tabular}{lcc}
\hline & $\mu[-]$ & $k\left[\mathrm{~mm}^{3} / \mathrm{Nm}\right]$ \\
polymers & 0.1 & $10^{-6}$ \\
$\mathrm{MoS}_{2}$ & 0.02 & $10^{-7}$ \\
graphite & 0.8 & $10^{-3}$ \\
ceramics & 0.5 & $10^{-6}$ \\
diamond & 0.8 & $10^{-3}$ \\
\hline
\end{tabular}

Table 3.1: Typical CoF values, $\mu$ and volumetric wear coefficient, $k$ of different materials, sliding against steel and measured in inert atmosphere [50] [64]

\subsection{Operating conditions}

The operating conditions of a piston compressor used in cooling systems are characterized mainly by thermal loads and the specific environment. In many cases, Stirling cryocoolers are hermetically sealed systems with working gas, i.e. helium or hydrogen. Such an environment will restrict the possibilities of chemical reactions and therefore the formation of protecting films on the surface, e.g. oxides. The working fluid, because of its specific chemical and/or physical properties, may have a dominant effect on tribological performance.

In this work a ringless piston is considered where the sliding contact is formed between two conforming surfaces. Typical contact pressures at the piston interface as reported are approximately $1.5 \mathrm{MPa}$ [13]. In case of rough surfaces, the load will be carried by a large number of small contacts, the so-called microcontacts. The real contact area over which two surfaces are in contact is typically only a few percent of the nominal contact area (the apparent contact area). Therefore the real contact pressure at the microcontacts level is considerably larger than the nominal contact pressure.

Two heat sources can be distinguished that contribute to the temperature rising of the sliding components; the convective heat transfer from the compressed hot gas and the heat generated due to frictional sliding. The gas generated heat flux is experimentally determined and is lower than $0.8 \mathrm{MW} / \mathrm{m}^{2}$ [56]. According to our numerical model the maximum surface temperature will be lower than $200{ }^{\circ} \mathrm{C}$ with the maximum heat flux that will occur at the top dead center (TDC). The friction generated heat input will reach its maximum at the half piston stroke when the sliding velocity $v$ is the highest. The rise in temperature of a sliding surface due to frictional heat depends on the frictional power,

$$
P_{\mu}=F_{\mathrm{n}} \mu v
$$

and the interaction of several factors, such as the real area of the sliding contact, the specific heat of the material, the thermal conductivity of the material, the temperature and volume of the surrounding material, and provided cooling. If the heat conduction from the surface to the bulk is hindered, for example by insufficient thermal conductivity, a high temperature will arise in the contact area. Consequently, thermal degradation of the surfaces of the contacting bodies can occur when a critical temperature is exceeded. This critical temperature can be a phase transformation 
or melting temperature for metals or graphitization in carbon coatings. Besides the formation of a high equilibrium temperature at the interface, also at the microcontact scale high temperatures can occur at the interface between contacting bodies. In such microcontacts, high local temperatures are acting at the asperity tops during only a short instant of time, also called flash temperatures. Flash temperatures can cause or initiate (local) thermal degradation. From the above discussion it can be concluded that with lower surface roughness the real contact area is larger. This will minimize the mechanical and thermal loads on the micro level.

\subsection{Requirements to the system}

Lifetime and efficiency of Stirling coolers depend to a great extent on the performance of the compressors. Specifically surface wear and sealing ability at the piston/cylinder interface is paramount. A minimum operating time of 10,000 hours is required with possibly the highest efficiency and reliability. From the tribological point of view this can be realized if a volumetric wear coefficient $k$ of sliding couples, lower than $5 \times 10^{-9}$ $\mathrm{mm}^{3} / \mathrm{Nm}$ can be demonstrated under operating conditions as expected in a piston compressor. This is a very low amount as compared to typical systems see table 3.1. The estimate is obtained based on the assumption that the amount of material loss is acceptable regarding efficiency, i.e. $1 \mu \mathrm{m}$ for a piston of $50 \mathrm{~mm}$ in diameter and length working with a rotating speed of $1500 \mathrm{RPM}$, piston stroke of $52 \mathrm{~mm}$ and contact load 1 N. Additionally, using self lubricating coatings instead of oil lubrication means that all the tasks of oil, for instance transport of wear debris and heat out of the contact have to be achieved by the coating in the absence of a fluid.

Important properties in different zones regarding tribology in a piston/cylinder assembly are presented in figure 3.2. The designed system should fulfil specific requirements, such as already discussed 1) good wear and friction characteristics, but also 2) strength and high dimensional stability under temperature variations, 3) high heat conductivity, regarding the heat sources present in the piston compressor, the surface and components material need to conduct heat efficiently reducing its operating temperature, 4) favorable heat expansion of the components, 5) low mass forces (piston) are required to minimize the dynamic forces. Typical piston materials are light alloys, cast irons and alloyed steels while cylinders are usually made from cast iron and alloyed steels. Furthermore, regarding manufacturing processes, the following remarks can be made. The sliding surfaces must be produced with a low surface roughness. Typically the higher the surface roughness the higher friction and wear occur in the initial stage of sliding (the running-in process). The running-in process is very critical regarding future performance and the lifetime of the compressor unit. This process is characterized by initial transfer film formation and strong reduction in friction. Wear rates are usually the highest during the running-in stage and sometimes unpredictable leading to a premature failure.

It is well known that mainly the top surface parameters control friction and wear. Modern coatings and surface treatment methods give the possibility to design material 
properties localized where they are most needed. Therefore the substrate material can be made from a material of desired mechanical and/or thermal properties while the coating is designed for obtaining low friction, resistance to wear, resistance for thermal loads, fatigue etc. A number of possibilities exists; they can be divided into two groups: 1) modification of the existing surface with thermal and/or chemical methods, leading to higher hardness gradually decreasing towards the core material, 2) coatings deposition, a different material offering better properties. In case of coatings we can expect sharp changes of material properties in one step or more (multi-layer coatings) leading to internal stresses. Coatings are deposited by means of different processes. External sliding surfaces of machine components are coated relatively easily (e.g. piston sliding surfaces). When internal surfaces (cylinder) need to be coated the coating thickness depends on the ratio between characteristic inner dimension to surface length. A limiting factor for the choice of a deposition process is the process temperature. Certain machine parts materials are sensitive to high temperature. This is because high temperature may lead to a material structure change (e.g. phase transformation, annealing, precipitation) and consequently to the material degradation.

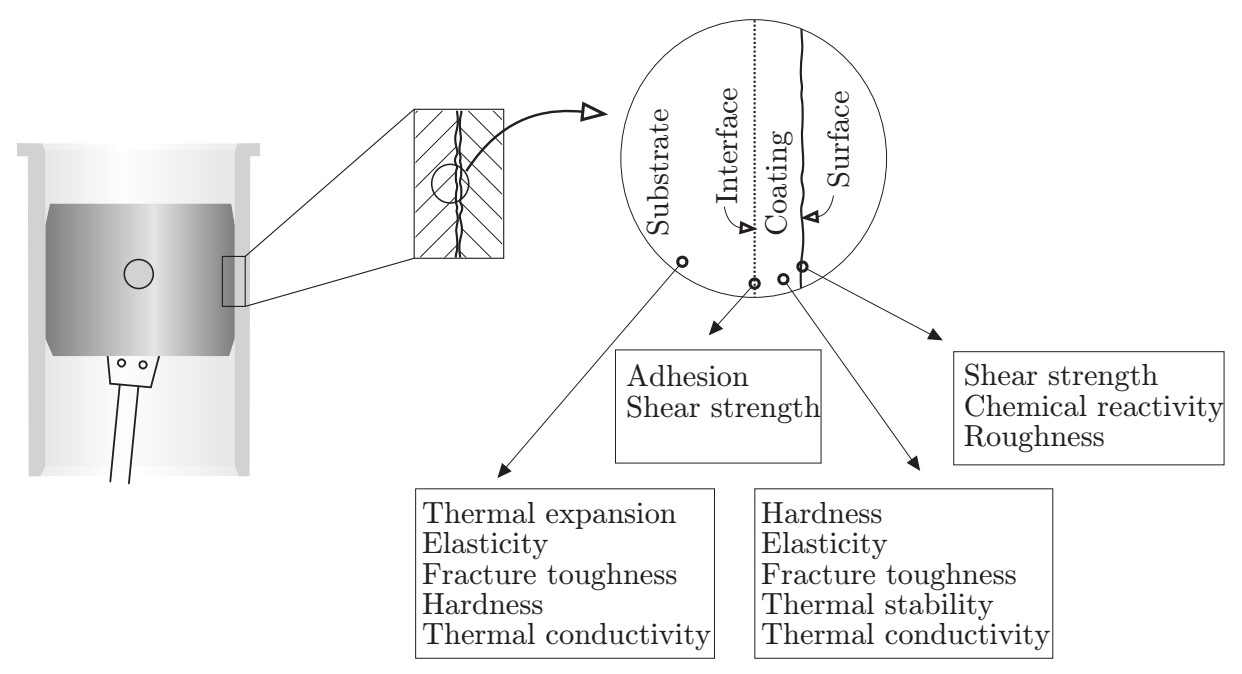

Figure 3.2: Piston/cylinder assembly and important material and surface properties in different zones.

\subsection{Candidate surface treatments}

The applicability of a wear protective coating or surface treatment for machine components is mainly the combination of high wear resistance and low coefficient of friction. Also the wear of the uncoated counter surface is important. There are 
different opinions on what can be considered as a low coefficient of friction. Typically for dry sliding components under ambient conditions a coefficient lower than 0.2 is considered to be low, ultralow approximately 0.1 and superlow below 0.01 .

\subsubsection{Surface hardening}

Various techniques exist for surface hardening, the most popular techniques are thermal and chemical hardening. In thermal hardening the existing metallurgical subsurface structure is modified due to high thermal gradient by means of quenching. Depending on the method refined grains and harder microstructure can be obtained. The other technique may involve change in chemical composition on the surface and subsurface material. An example is carburizing and nitriding where carbon or nitrogen enters the surface by a diffusion process. Thermo-chemical hardening is also widely used. However, typically in thermal processes high temperatures and material phase transitions are involved. For high precision components often problems with shape distortion arise due to the high process temperatures and residual stresses. Correction by grinding is necessary afterwards. This has obvious consequences for the manufacturing precisions and costs.

\section{Kolsterising ${ }^{\circledR}$}

A number of low temperature hardening processes have also been developed. The Kolsterising process from Bodycote Metal Technology Group/Apeldoorn has shown good promise as a surface hardening treatment for austenitic stainless steel alloys applied in unlubricated piston pumps on an industrial scale. According to Bodycote's promotional information [78], the Kolsterising process is based on carbon diffusion into the surface of an austenitic stainless steel at low temperature, $<300{ }^{\circ} \mathrm{C}$, from a gaseous atmosphere. The amount of carbon introduced is $6-7 \mathrm{wt} \%$ at the surface, decreasing to zero at a depth that depends on the time of the treatment. Bodycote's regular treatment provides a hardened material up to about $33 \mu \mathrm{m}$. Within the material, carbon is incorporated in supersaturated interstitial solid solution in the austenitic phase. Accommodation of the carbon in the layer is claimed to cause expansion of the affected austenite crystal lattice. This imposes compressive stresses in the layer. These stresses, combined with the changes in chemical composition, significantly harden the material. Hardness values $\mathrm{HV}_{0.05} 1000-1200{ }^{1}$ are produced at the shallow surface and decrease with depth to the original substrate hardness of about 200 at $30-40 \mu \mathrm{m}$. Constant hardness is obtained on the entire surface independent of geometry or shape. Due to the low hardening temperature there is no change in shape or size and the material retains its original structure. The hardened surfaces were tested against surface fatigue showing superior performance. In [30] some experimental data can be found. Details of the actual carburization treatment are proprietary.

\footnotetext{
${ }^{1}$ Vickers hardness scale, measured with low load applied on the indenter only $0.05 \mathrm{~N}$ to avoid the influence of softer sub-material
} 


\subsubsection{Soft coatings}

\section{Polymer coatings}

Polymers are frequently used for dry sliding components because of their selflubricating properties. Such polymers exhibit low shear strength and the material in contact deforms readily during sliding. The lubrication action of polymers occurs via material transfer to the counter surface during relative motion. A thin transfer film of polymer material is produced and this film reduces the CoF. The tribological performance of commercially available PTFE-based coatings was evaluated in environments simulating compressor conditions used in refrigeration systems [15] [16]. It was found that their behavior was not greatly affected by the environment and under certain conditions surpassed Diamond Like Carbon coating showing a very high load carrying capacity and 'gradual' failure. Mainly the wear debris generated acted as a third-body lubricant preventing catastrophic failure. The results show that a coefficient of friction of 0.1 is the lower limit.

The contact surface temperature between sliding parts usually limits the use of polymers. To improve the thermal stability as well as mechanical and tribological performance, polymer coatings are often filled with other materials. Composites are made by adding solid lubricants to the matrix such as graphite or $\mathrm{MoS}_{2}$. As an example, Torlon 4301 is a polyamide-imide resin designed as a general purpose, low friction and low wear material. The resin composition contains $12 \%$ graphite and $3 \%$ fluoropolymer. Graphite conducts heat very well, thus efficiently reduces the contact surface temperature.

\section{Lamellar coatings}

Molybdenum disulfide $\left(\mathrm{MoS}_{2}\right)$ and graphite are the most popular lamellar coating materials. $\mathrm{MoS}_{2}$ has a lamellar structure with individual sheets of molybdenum and sulphur atoms. The material has a strong structure in two dimensions but it is weak in the third. It shows one of the lowest CoF (0.02) measured for dry sliding contact. Good adhesion and a low $\mathrm{CoF}$ has been achieved with sputtered $\mathrm{MoS}_{2}$ films and typically only a very thin film of about $0.2 \mu \mathrm{m}$ is required for effective lubrication. $\mathrm{MoS}_{2}$ is considered as the best solution for vacuum solid lubrication. The film thickness, substrate material, surface roughness and the environment, especially humidity, have a considerable influence on the tribological properties. Unfortunately performance of $\mathrm{MoS}_{2}$ is dependent on the sliding motion. Under reciprocating sliding the individual sheets are gradually removed from the sliding contact. Therefore, the wear coefficient measured during reciprocating sliding is high. Graphite, however, relies on the presence of vapors to give low shear strength between its individual planes. Those materials are considered as not being effective lubricants in Stirling coolers. 


\subsubsection{Ceramic coatings}

Ceramics are well known as wear protective materials. Titanium nitride (TiN) and chromium nitride $(\mathrm{CrN})$ are the most popular ceramic coatings. Thin, hard coatings can be produced by PVD or CVD methods. Because of high temperature and corrosion resistance they are often applied in piston engines. Those coatings have been intensively investigated and are currently in use [5] [43] [52] [85]. Ceramic coatings perform well under oil lubricated sliding conditions. Unlubricated sliding turned out to be detrimental for the counter sliding surface. Mainly due to abrasive wear resulting from very hard wear debris formed during sliding. Ceramic inclusions embedded in DLC matrix are already applied on an industrial scale. These coatings have low residual stress, high hardness, and high toughness and exhibit very good tribological properties.

Coefficient of friction of ceramics sliding in vacuum is rather high and spans from 0.3 to 0.9 [75]. The idea of minimizing friction in zirconia by doping with copper oxide $(\mathrm{CuO})$ was investigated by Pasaribu [70]. He concluded that the addition of $\mathrm{CuO}$ provides a soft interfacial layer in the contact that can reduce friction from 0.8 to 0.2 depending on operating conditions.

\subsubsection{DLC coatings}

Thin Diamond-Like Carbon (DLC) coatings have proved to provide excellent low friction and wear protection properties in dry sliding conditions. The CoF values measured, when sliding against different metallic or ceramic counterparts, are typically in the range of 0.2 to 0.02 [6] [8]. This is in the same range or lower than the typical values in oil lubricated sliding conditions. These values are considerably lower than the friction values using other hard surface coating. At the same time, DLC coatings also provide an excellent wear resistance, which has proved to be far better than, for example, the wear resistance of ceramic coatings [50] [73]. The application of DLC coatings will be further investigated here.

\subsection{Properties of DLC coatings}

Carbon forms various crystalline and disordered structures because it is able to exist in different hybridizations $\left(\mathrm{sp}^{1}, \mathrm{sp}^{2}, \mathrm{sp}^{3}\right)$ [72]. According to different carbon synthesis methods and deposition parameters the carbon coatings differ with the percentage of carbon allotrope content. They can be more diamond-like with a large percentage of $\mathrm{sp}^{3}$ bonded carbon atoms (responsible for high hardness, wear resistance and thermal conductivity) or graphite-like with a higher percentage of $\mathrm{sp}^{2}$ carbon bonds (low CoF). Polycrystalline diamond (PCD) and amorphous diamond (DLC) are two specific forms of carbon coatings. Carbon occurs in them as a structured diamond and/or graphite allotrope or as amorphous carbon. Amorphous carbon (a-C) is a disordered structure where the $\mathrm{sp}^{3}$ and $\mathrm{sp}^{2}$ carbon bonds are both randomly present. 
Typically, DLC coatings contain a percentage of hydrogen originating from the gas that provides the carbon. The amount of hydrogen stored in the structure depends on the deposition technique. Amorphous hydrogenated carbon coatings are denoted as a-C:H and contain usually 10-40\% of hydrogen. During the deposition process very often some metals are doped into the structure. The incorporation of metals or silicon improves mechanical and tribological properties [20]. More information on the deposition methods can be found in Appendix D.

\subsubsection{Mechanical properties}

The mechanical properties of DLC coatings vary widely depending on their deposition method, structure and chemical composition. Generally they combine ceramiclike properties, high hardness, high internal stresses and polymer-like relatively low elastic modulus. Therefore they exhibit exceptionally high hardness/Young's modulus $(H / E)$ ratios, with values higher than 0.08 up to 0.2 . This high ratio means the DLC materials are relatively strain tolerant. Donnet and others [19] measured mechanical properties of various DLC coatings of different hydrogen content. They stated that at high hydrogen content the a-C:H coatings can be more viscoplastic in nature. It has been suggested that the specific structure of these materials implies that in such structures there is a possibility for the random carbon network to relax, probably through free volumes like in polymers. It has been concluded that the network relaxation permits weaker interaction between asperities on the sliding surfaces. The friction reduction is correlated with the measured viscoplastic properties [31]. By comparison, the highest achievable $H / E$ ratio for tool steel is about 0.02 . Typically for ceramics this value is around 0.06 . Increasing hydrogen content has a tendency to reduce both the hardness and Young's modulus [32]. On the other hand increasing $\mathrm{sp}^{3}$ fraction improves the mechanical properties.

A thick coating is required for various applications, in particular, to improve the machine components lifetime. Coating thickness available on the market are typically up to $4 \mu \mathrm{m}$ [6] [8] [20]. In practice the thickness is closely related to internal stresses generated during deposition, the coatings density and hardness. With increasing coatings thickness higher internal stresses are introduced. This subsequently lowers coating adhesion resulting in a lower wear resistance and the coating has a tendency to spall from the substrate. Internal stresses formed during deposition have two sources. Thermal stresses, caused by the difference in thermal expansion between the coating and substrate. The stresses are generated during cooling from the process temperature to room temperature. The second source of stresses in the coating is caused by growth of the coating during deposition. The material volume of the coating changes during coatings deposition. However, the coatings growth is restricted because it is adhered to the substrate. Prevention of volume change gives rise to compressive internal stresses. For tribological performance the internal stress can be advantageous. High compressive stresses in general improve resistance against fatigue wear [44]. However, when too high it will cause low adhesion or form cracks in the substrate or at the interface. 
The adhesion strength between the coating and substrate material is determined by the kind of bonds that occur on the interface (dependent on physical, chemical and mechanical interactions). The coating adhesion is often improved by depositing a thin interlayer on the substrate surface. An example is the deposition of a thin Ti layer. Titanium is a strong carbide former which adheres strongly to the substrate. DLC coatings typically have high hardness and wear resistance but they have also high internal compressive stresses [36], which in turn limit the thickness of the coating, because of adhesion failure. Often metal nano-particles are introduced into the DLC structure (Me-DLC) which markedly reduce compressive stresses thus thicker coating can be deposited [45]. The better adhesion of the Me-DLC coatings is the key issue why these coatings have found large application in industry [41].

\subsubsection{Tribological aspects}

DLC coatings are known to exhibit excellent properties concerning friction and wear under specific conditions [4] [20]. The lubrication action does not involve material deformation as in the case of polymers and lamellar coatings. Shear deformation is not visible in DLC coatings because of their amorphous structure, they possess no crystallographic shear planes to enable easy shear. The DLC coatings slide through a sharp interface between the coating itself and counter surface which may be covered by a very thin layer of transfer material [20]. The CoF may vary in the range of 0.001 to 0.7 depending on test conditions and environment. Two parameters are reported often having the most influence on the tribo-properties which are: a) intrinsic, coating specific, the friction and wear behaviors of the coatings are strongly affected by their chemical and structural nature and b) extrinsic, the tribo test conditions specific, i.e. contact pressure, nature of motion, sliding speed, substrate material support and adhesion, chemistry and temperature of the environment, nature of the counter surface. The specific contributions to friction of DLC coatings will be discussed next.

\subsubsection{Physical and mechanical interactions}

In general when rough surfaces slide, a high level of mechanical interlocking takes place between surface asperities which leads to high frictional losses. This effect is pronounced during the running-in stage of sliding. In fact, recent systematic studies confirm the existence of almost linear correlation between surface roughness and friction and wear coefficients of sliding diamond surfaces [4]. Due to the amorphous structure the roughness of the coating is usually low. The DLC coatings follow the roughness of the substrate with some tendency to smooth the surface roughness depending on the coating thickness. This is in contrast to the pyramidal structure of some PCD surfaces. However, roughness of DLC coatings may increase depending on the deposition process due to formation of numerous nano/micro particles and/or droplets being ejected from the solid carbon source [27].

Adhesion is considered as the dominant contribution to friction of DLC coatings provided that the sliding surfaces are relatively smooth. The adhesive interaction 
may primarily result from several types of bonding. Among others, the covalent bonds interaction between unoccupied or dangling $\sigma$-bonds of sliding carbon interfaces can account for a significant source of adhesion. Covalent bonding is the strongest type in carbon-based materials. Apparently under inert sliding conditions the covalent bonds that are free and active interact with each other and give rise to high $\mathrm{CoF}(>0.7)$ as reported in [23] [21]. However, the $\sigma$-bonds react readily with environment $(\mathrm{O}, \mathrm{H}$, $\mathrm{OH})$. Adhesion with DLC coatings is not likely to involve covalent $\sigma$-bonds if $\mathrm{O}, \mathrm{H}$ or $\mathrm{OH}$ are available in the test atmosphere [20].

\subsubsection{Environmental effects on the friction}

Among the gaseous species, oxygen and water molecules have been shown to have the strongest effect on friction and wear of DLC coatings. In vacuum and inert gasses a CoF of less than 0.01 is feasible, especially with highly hydrogenated DLC coatings. However when oxygen and/or moisture are added into the sliding atmosphere the CoF may increase substantially.

Tribochemistry between hydrogen and the carbonaceous network of the DLC film has been shown to be responsible for the control of a superlow friction regime observed with a-C:H coatings [20]. In inert or vacuum conditions, friction and wear can be accurately controlled by hydrogen. This effect is explained more in Appendix E. Low hydrogenated coatings ( $<34$ at \% hydrogen) may exhibit a similar superlow friction level by introducing a significant pressure of hydrogen in the friction environment. The preservation of superlow friction of a-C:H was investigated under different hydrogen pressures [31]. At low hydrogen pressure (50 Pa) similar behavior effects were observed as in vacuum, meaning strong adhesion between counter surfaces leading to a local loss of transfer layer. At a hydrogen pressure above $500 \mathrm{~Pa}$ the transfer film is mainly preserved and low CoF $(<0.01)$ has been measured. These findings suggest that both, the specific features of a-C:H itself and the combination with the atmosphere define the super low friction. Without those conditions, friction reaches high values, consistent with the tribological behavior of diamond in inert or vacuum atmosphere. Thus, hydrogen is essential to achieve an accurate control of the friction of DLC coatings.

Under conditions where the DLC coating was present only on one of the sliding surfaces, the formation of a carbon rich transfer layer on the counter surface was paramount for achieving ultra-low friction [26] [63] [25] [21]. The type of composition of the counter face material may play some role in formation of the transfer layer. Normally, those materials that are known to be very strong carbide formers ( $\mathrm{Ti}$, $\mathrm{Fe}, \mathrm{W}, \mathrm{Si}$ etc) tend to generate such layers faster and with a much higher degree of coverage and strength bonding. During sliding also wear debris will be produced from the contacting surfaces. These debris particles are trapped at the sliding contact interfaces and undergo severe physical grinding action as well as chemical reaction with the uncoated surface. Often the debris particles are smeared on one or both sliding surfaces. 


\subsection{Experimental investigation}

Mechanical and tribological characterizations were performed on four sets of DLC coated samples. The DLC coatings are commercially available and delivered by Bekaert Advanced Coatings /Belgium (coatings named 26A and 27A) and Hauzer Techno Coating BV /Netherlands (H1 and NFC). Characteristic data of the coatings according to the producer specifications and mechanical properties measured with nano-indentation technique are given in table 3.2. The tribological performance was evaluated in environments simulating compressor conditions. A special tribometer was developed for this purpose, details are given in Appendix F.

\begin{tabular}{lcccc} 
& $26 \mathrm{~A}$ & $27 \mathrm{~A}$ & $\mathrm{H} 1$ & $\mathrm{NFC}$ \\
\hline structure & multilayer & multilayer & multilayer & singlelayer \\
thickness $[\mu \mathrm{m}]$ & $1.5-2.0$ & $1.5-2.0$ & $2.3-2.5$ & 1.2 \\
adhesion $[\mathrm{N}]$ & 20 & 20 & 50 & - \\
hydrogen [at \%] & $25-30$ & $25-30$ & - & - \\
\hline \hline hardness [GPa] & 25.201 & 23.584 & 27.086 & 9.458 \\
E-modulus [GPa] & 193.246 & 206.094 & 237.030 & 70.390 \\
H/E rate [-] & 0.1304 & 0.1144 & 0.1142 & 0.1343 \\
\hline
\end{tabular}

Table 3.2: Coatings data according to producer and measured mechanical properties

Pin-samples are cut from large bearing balls $(76.2 \mathrm{~mm}$ in diameter) made from the commonly used bearing steel, AISI 52100. The hemispherical surface which is the sliding surface was coated. The large ball diameter was chosen to maximally reduce the contact pressure towards the one expected on the piston sliding surface. Roughness measurements were taken using an interference microscope. The averaged surface roughness measured was $\mathrm{Ra}=0.2 \mu \mathrm{m}$. As depicted, some holes and scratches are visible which can be as much as $3 \mu m$ deep, see figure 3.3 .

a)

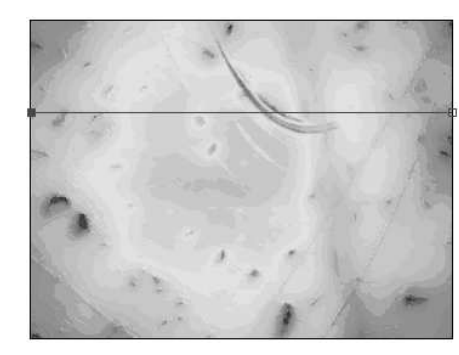

b)

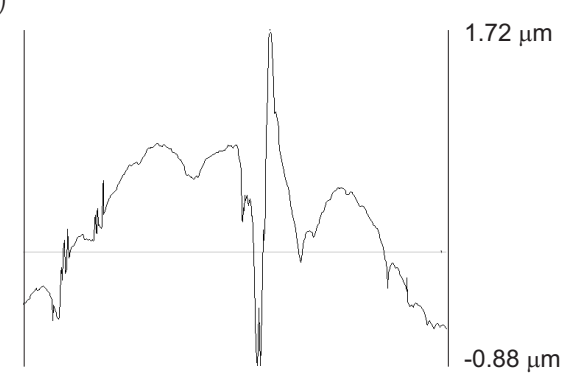

Figure 3.3: a) Initial pin surface roughness, $\mathrm{Ra}=0.2 \mu \mathrm{m}$, measured with the Micromap surface profilometer and b) surface profile along the black line 
a)

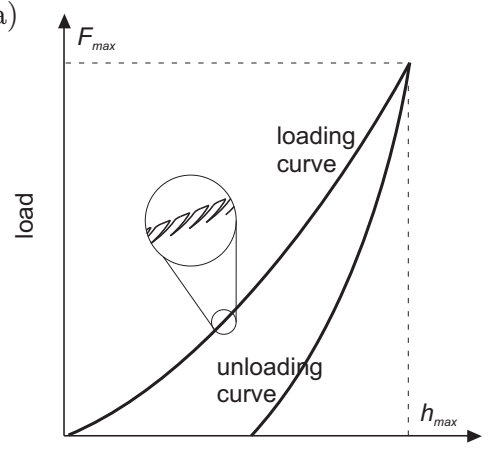

indentation depth

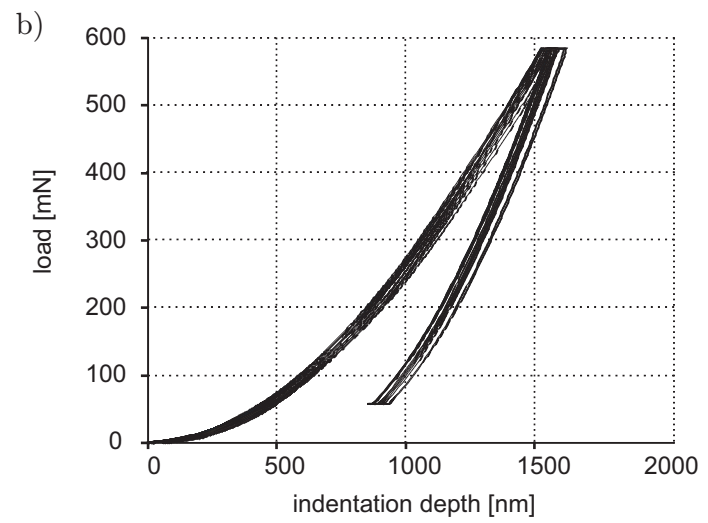

Figure 3.4: a) Schematic illustration of the CSM nano-indentation test, b) force displacement curves from nano-indentation tests on the $27 \mathrm{~A}$ coating

Discs were made from two types of steel. The discs made from $316 \mathrm{~L}$ were surface hardened to approximately $1400 \mathrm{HV}_{0.05}$ according to the Kolsterising process (further called $316 \mathrm{~L}+\mathrm{K})$. Before surface hardening the disc surfaces were polished to roughness $\mathrm{Ra}=0.2 \mu \mathrm{m}$. A second set of discs was prepared from AISI 52100 bearing steel (further called 52100).

\subsubsection{Nano-indentation measurements}

The mechanical properties of a material are usually measured by making a plastic indentation on the surface with an indenter of well-defined geometry and material. For coatings usually the Berkovich type, diamond indenter is used. The apparatus applies the load and measures the displacement (indentation depth). Using an indenter tip with a calibrated area function and based on the unloading part of the loaddisplacement curve, both hardness $H$ and elastic modulus $E$ can be determined. The hardness is defined as the pressure under the tip, given by the ratio of force to the projected area of plastic deformation. The shape of the unloading curve provides a measure of elastic modulus. The theory of determining hardness and elastic modulus from a load displacement curve is given in [18]. The measurement in a conventional way (quasi-static mode) has the disadvantage that it calculates the mechanical properties based on one point of the load-displacement curve and the maximum indentation depth corresponding to $F_{\max }$ point, see figure 3.4 . Regarding hardness measurements of coatings the indentation load must be chosen carefully. The coating thickness is usually lower than $4 \mu \mathrm{m}$. When the applied load is too high the measured value is influenced by the substrate material. 
a)

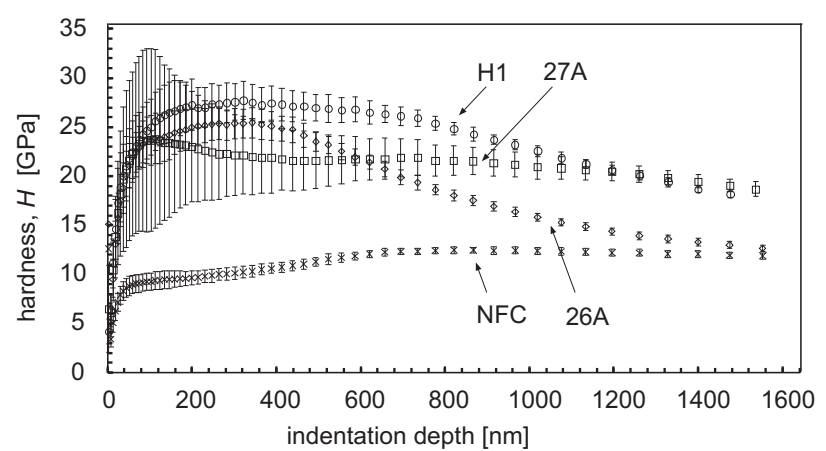

b)

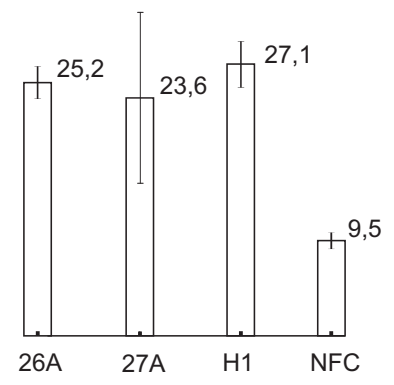

Figure 3.5: a) Hardness vs indentation depth and b) averaged values from data at $10 \%$ of the maximum indentation depth with a standard deviation

a)

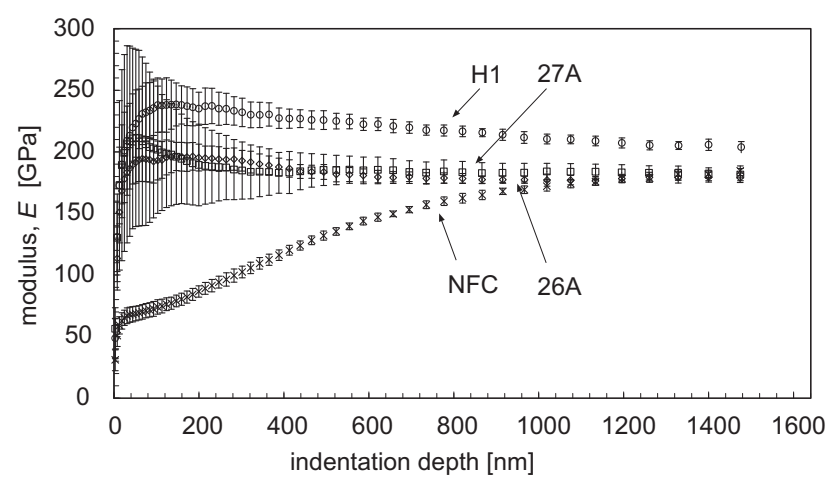

b)

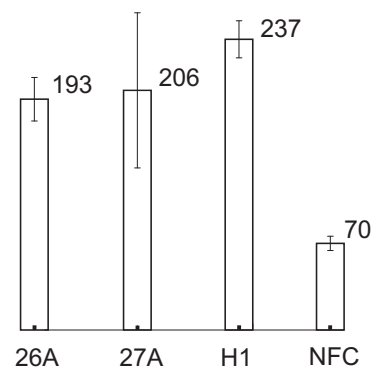

Figure 3.6: a) Elastic modulus vs indentation depth and b) averaged values from data at $10 \%$ of the maximum indentation depth with a standard deviation

\section{Measurement results}

With a dynamic mode the risk of sensing substrate material is excluded. The Continuous Stiffness Measurement (CSM) technique allows the contact stiffness to be measured continuously along the loading portion of the test, thus the values of $H$ and $E$ are determined as a function of the coating thickness. The CSM system applies a load to the indenter tip to force the tip into the surface while simultaneously superimposing an oscillating force with a force amplitude generally several orders of magnitude smaller than the nominal load. The indentation system and contact together are modeled as a simple harmonic oscillator. Thus, by monitoring the ratio between force and displacement oscillation amplitudes, as well as phase shift between the two, the elastic stiffness of the contact may be evaluated continuously as the 
indenter penetrates the material [66].

Hardness and elastic modulus of the examined coatings were evaluated according to the CSM procedure with the Berkovitch indenter. The coatings were deposited on AISI 52100 steel. The results are presented on the figures 3.5 and 3.6. According to a standard procedure, one test consists of 9 indents. Due to a large scatter in the measured data the samples: 26A, H1, NFC were tested twice. The abrupt increase of the values at the initial stage may be due to some factors including water adsorption, film surface roughness, elastic deformation from both the tip and the sample surface and the intrinsic error of the instrument [62]. In general it reaches a small plateau and finally becomes very close to the value of the substrate. The measured hardness of the NFC coating was lower than that of the substrate material. The sample 27A showed the largest spread of measured data therefore it was measured three times. The recorded force displacement curves of the sample $27 \mathrm{~A}$ are shown in figure $3.4 \mathrm{~b}$. The coating hardness and elastic modulus values are averaged from data at $10 \%$ of the maximum indentation depth. For determination of the elastic modulus Poisson's coefficient $\nu=0.25$ was assumed.

\subsubsection{Friction and wear measurements}

The coatings friction characteristics were evaluated in a pin-on-disc tribometer in an environmental chamber where the gaseous environment can be controlled. Pin-on-disc testing procedures are most frequently used in laboratory sliding and wear tests. The vacuum tribotester was developed in the Surface Engineering and Tribology Group. Details about the vacuum tribometer can be found in Appendix F.

Prior to experiments the samples were cleaned in an ultrasonic bath for at least 10 minutes and rinsed with alcohol. In order to protect the sliding surfaces from any contamination all operations during mounting were done using gloves. The experiments were run under contact load of $1 \mathrm{~N}$. With the pin sphere radius 38.1 $\mathrm{mm}$ it results in the mean and maximum Hertzian contact pressure of respectively $80 \mathrm{MPa}$ and $120 \mathrm{MPa}$. Regarding the surface irregularities as shown in figure 3.3 much higher contact pressures can be expected locally. Some tests were done with higher contact loads in order to check the coatings sensitivity. Before experiments the chamber was evacuated to $0.1 \mathrm{mPa}$ prior to filling with helium He5.0. The tests were run for $10 \mathrm{~km}$ or terminated when the $\mathrm{CoF}$ reached values higher than 0.3 . Some tests were done with extended sliding distance to $25 \mathrm{~km}$ to investigate the coating performance at a long sliding distance.

The measured friction coefficients and estimated volumetric wear coefficients from all experiments are summarized in table 3.3 . 


\begin{tabular}{lccccc} 
& & $26 \mathrm{~A}$ & $27 \mathrm{~A}$ & $\mathrm{H} 1$ & $\mathrm{NFC}$ \\
\hline$\mu$ & & - & 0.020 & 0.010 & 0.003 \\
$k$ & in helium sliding on 316L+K & - & $9.2 \mathrm{e}-8$ & $9.2 \mathrm{e}-8$ & $2.5 \mathrm{e}-10$ \\
$R$ & & $0 / 4$ & $3 / 4$ & $2 / 4$ & $2 / 4$ \\
\hline$\mu$ & \multirow{2}{*}{$\begin{array}{c}\text { in helium sliding on 52100 } \\
k\end{array}$} & - & - & 0.012 & 0.010 \\
$R$ & & - & - & $3.9 \mathrm{e}-10$ & - \\
\hline$\mu$ & & - & 0.050 & 0.0012 & 0.040 \\
$k$ & in vacuum sliding on 52100 & - & $5.5 \mathrm{e}-9$ & $1.1 \mathrm{e}-8$ & $2.3 \mathrm{e}-8$ \\
$R$ & & - & $1 / 4$ & $2 / 4$ & $1 / 4$ \\
\hline
\end{tabular}

Table 3.3: The measured CoF, $\mu$ [-] under sliding velocity $1 \mathrm{~m} / \mathrm{s}$ and normal force 1 $\mathrm{N}$ obtained in helium and vacuum environment. The averaged $\mu$ value after runningin stage and the lowest measured volumetric wear coefficient, $k\left[\mathrm{~mm}^{3} / \mathrm{Nm}\right]$, is given. The $\mathrm{R}$ factor indicates the rate between the number of successful measurements to the total number of performed experiments

\section{Measurements on the Bekaert coatings}

Results of four experiments performed on the 27A samples are shown in figure 3.7. The test conditions are summarized in table 3.4. Varying CoF evolution during running-in can be observed, see figure 3.7b. During the experiment test 2 in the first $50 \mathrm{~m}$ a high $\mu=0.5$ was recorded: after that distance coefficient of friction rapidly decreased to 0.01 to finally stabilize at 0.02 .

\begin{aligned} & \hline normal load $1(2,3) \mathrm{N} \\ &$ sliding velocity $1 \mathrm{~m} / \mathrm{s} \\ &$ temperature $\mathrm{RT} \\ &$ disc material $316 \mathrm{~L}+\mathrm{K} \\ &$ disc roughness $\mathrm{Ra}=0.2 \\ &$ atmosphere helium \\ & sliding distance $10 \mathrm{~km} \\ &$\hline\end{aligned}

Table 3.4: Experimental conditions

In experiment, test3 initial $\mu=0.1$ gradually decreased to $\mu=0.02$ and until approximately $5 \mathrm{~km} \mu$ remains on that level. After $6 \mathrm{~km}$ the coefficient of friction started rising up to reach 0.035 and after $15 \mathrm{~km}$ its value dropped again to $\mu=0.02$. The initial decrease in CoF is explained by the transfer film formation from the pin to the disc surface. Evidence of the transferred material was observed under light microscope and is shown in figure 3.9. The experiment, test1 started with high $\mu$ and essentially remained on that level until the experiment was terminated by the operator. This friction characteristic basically shows an instant failure of the coating. Microscopic inspection revealed that the coating indeed had been removed from the sliding spot. 

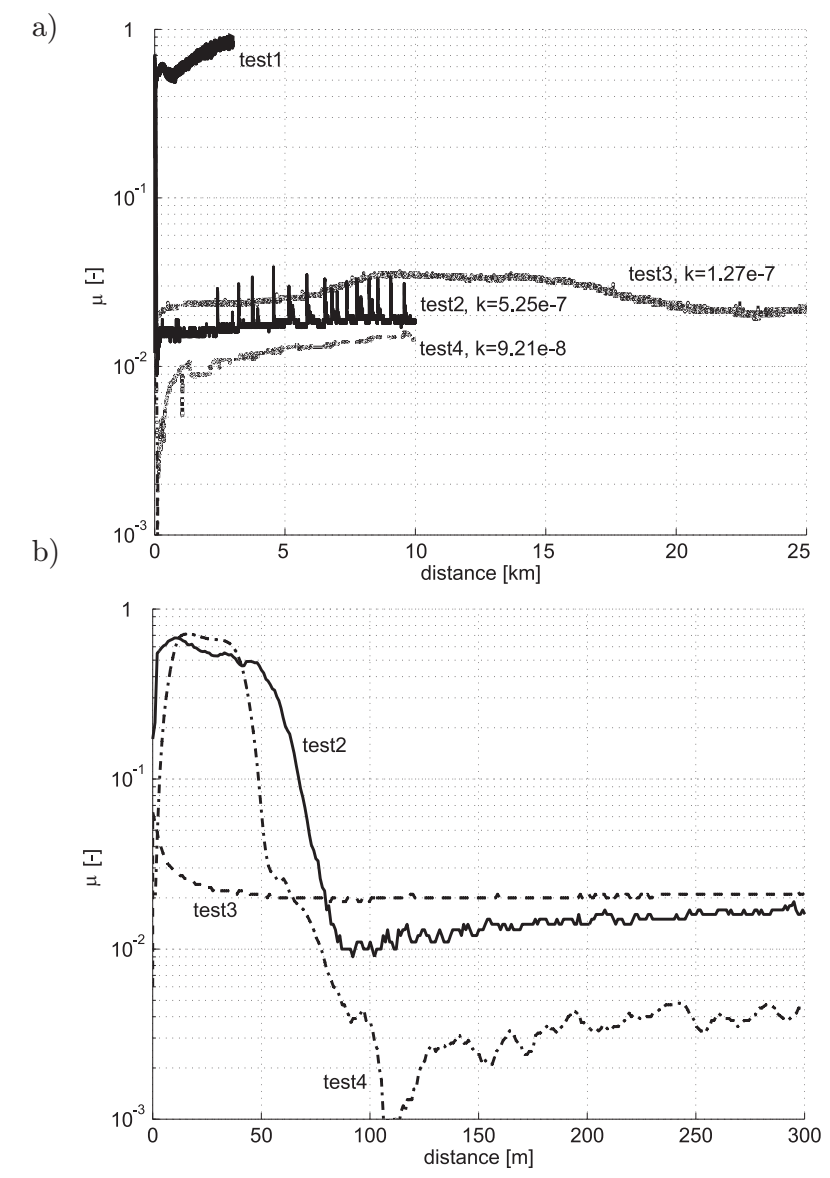

Figure 3.7: a) CoF measurements results, the sliding pair: coating 27A on $316 \mathrm{~L}+\mathrm{K}$ disc, b) the running in stage

The results show that the $27 \mathrm{~A}$ coating can run-in to a state of ultra-low CoF, showing repeatability in the experiments $3 / 4$ with a mean $\mathrm{CoF}$ in the steady state of 0.02 . All tests performed on the $26 \mathrm{~A}$ samples ended with premature failure and the results are not presented. Essentially their characteristics can be compared to the experiment test1. All samples tested with higher loads: 2 and $3 \mathrm{~N}$, have shown a high $\mu=0.6$. This high CoF indicates that the coatings failed. Microscopic inspection revealed that the coating indeed had been removed from the sliding spot.

Examples of wear measurements obtained with the interference microscope are presented in figure 3.8. There is a nonuniform material removal from the pin across the sliding spot, shown in figure $3.8 \mathrm{a}$ on the corresponding surface profile. In the figure $3.8 \mathrm{~b}$ no wear could be observed. The wear rates of the coatings were estimated based on the measured wear spots diameter. 
a)
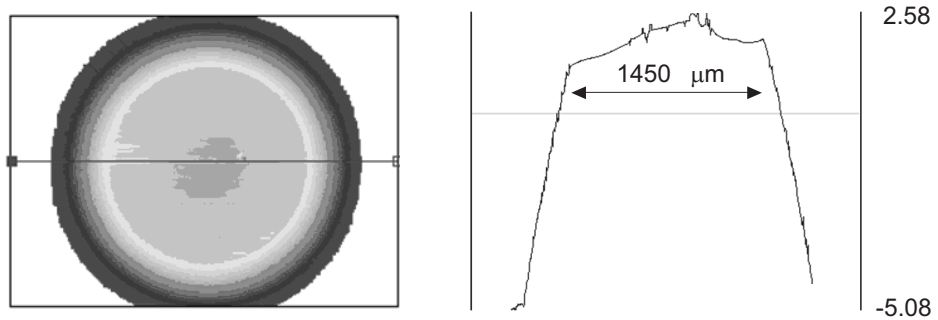

b)
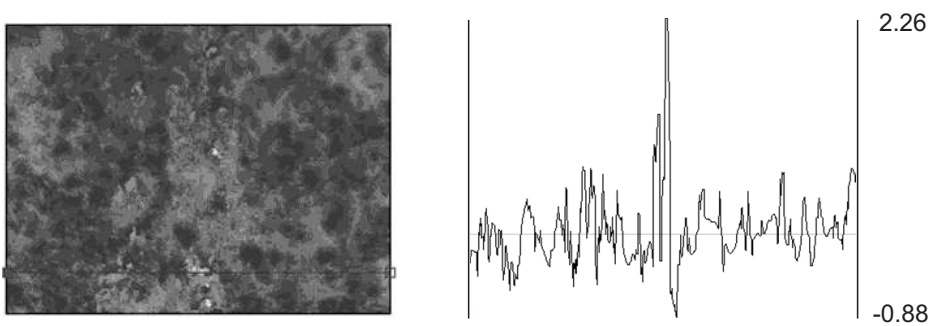

c)
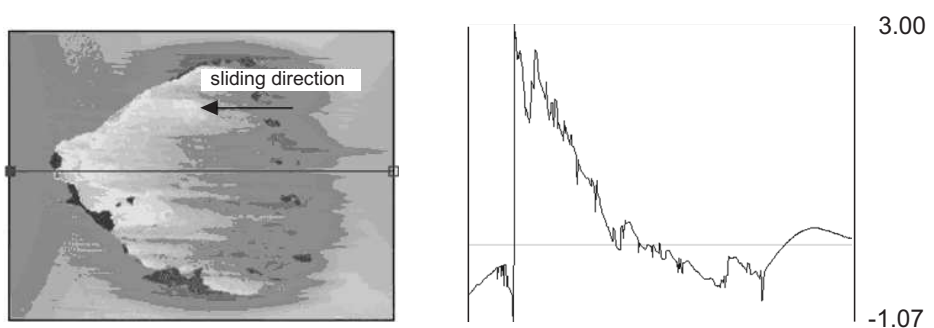

d)
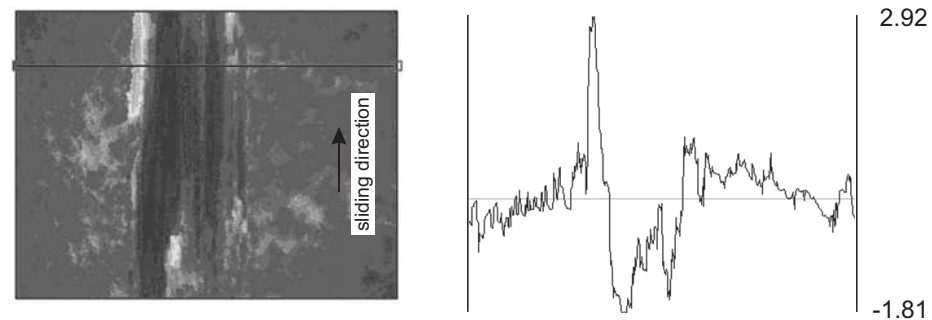

Figure 3.8: Interference microscope images (left) with corresponding surface profiles (right) from two tests, see figure 3.7. Figures a) and b) show the pin profile and the disc profile respectively after test $2, \mathrm{c}$ ) and d) show the pin profile and the disc profile after test1

Figure 3.9 shows the transfer material on the counter surface that was formed on the disc during the experiment test2. This image is a zoom-in on figure $3.8 \mathrm{~b}$. It 


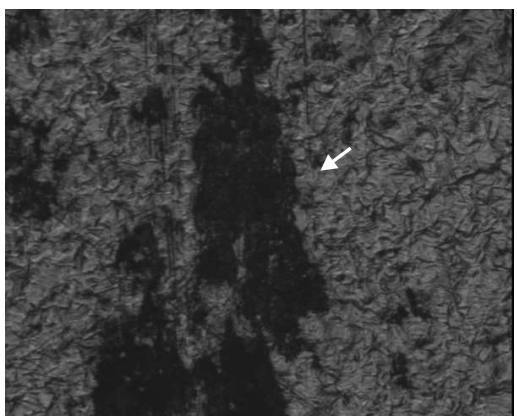

Figure 3.9: Traces of the transferred material on the disc surface, zoom-in image from figure $3.8 \mathrm{~b}$.

should be noticed here that the available amount of DLC material to be transferred from the pin sliding spot to the disc surface is relatively small compared to the disc surface that needs to be covered. As can be seen on the image the tribo-film does not entirely cover the sliding path. Most of the material is locked within the surface roughness. This limited amount of DLC was sufficient to form the tribo film and effectively reduce $\mathrm{CoF}$ to 0.02 over the sliding distance of $25 \mathrm{~km}$ (test3).

\section{Measurements on the Hauzer coatings}

\begin{aligned} & \hline normal load $1 \mathrm{~N} \\ &$ sliding velocity $1 \mathrm{~m} / \mathrm{s} \\ &$ temperature $\mathrm{RT} \\ &$ disc material 52100 and \\ & $316 \mathrm{~L}+\mathrm{K} \\ &$ disc roughness $\mathrm{Ra}=0.02 \\ &$ atmosphere helium \\ & sliding distance $10 \mathrm{~km} \\ &$\hline\end{aligned}

Table 3.5: Test conditions

After the first experiments discussed above showing relatively poor reproducibility of the coatings performance it has been decided to change the type of discs used. Because of the low surface roughness, discs made of 52100, from SKF/Netherlands were chosen. Surface roughness of the discs is ten times lower than the ones previously used. In the meantime the stainless steel discs were mirror polished to the same roughness as the 52100 discs and subjected again to the Kolsterising process. The test conditions are summarized in table 3.5 .

The results of CoF measurements performed on the DLC coatings delivered by Hauzer are presented in figure 3.10 the single layer, hydrogenated coatings named NFC and 
a)

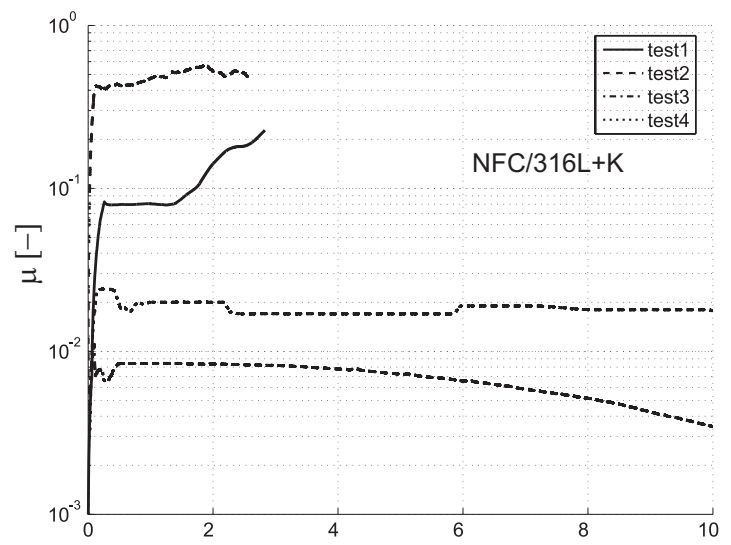

b)

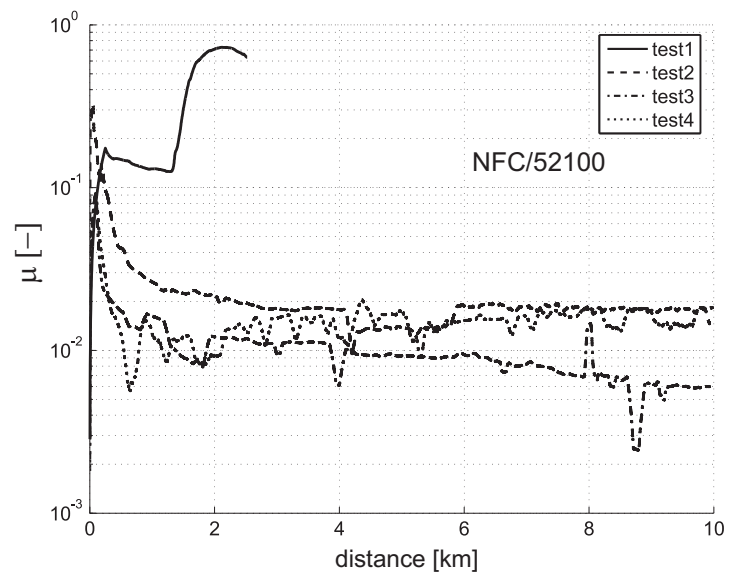

Figure 3.10: CoF of the sliding pairs: a) the coating NFC on $316 \mathrm{~L}+\mathrm{K}$ steel and b) the NFC coating on 52100 steel both measured in helium

in figure 3.11 the multilayer coating named H1. The presented data are filtered ${ }^{2}$. There is a clear difference when comparing the friction characteristics obtained with the different discs. The discs made of $316 \mathrm{~L}+\mathrm{K}$ generate a relatively smooth friction signal over the sliding distance. The discs made of 52100 give a more irregular signal with a more pronounced running-in stage. The running-in stage takes approximately $3 \mathrm{~km}$ after which the CoF stabilizes around 0.02. A similar trend can be seen for both types of coatings. In case of $316 \mathrm{~L}+\mathrm{K}$ discs the CoF stabilizes after already less than $1 \mathrm{~km}$. But reproducibility of the coating performance is lower here. The following explanation is offered. Since the surface roughness is the same for both discs, the difference in performance can be related to the surface material. Namely, the high carbon concentration on the Kolsterized surface may offer favorable conditions for the

\footnotetext{
${ }^{2}$ A standard Matlab function 'filter' was employed. It finds a running average of a chosen set of data (set of 100 data points was used)
} 
a)

b)
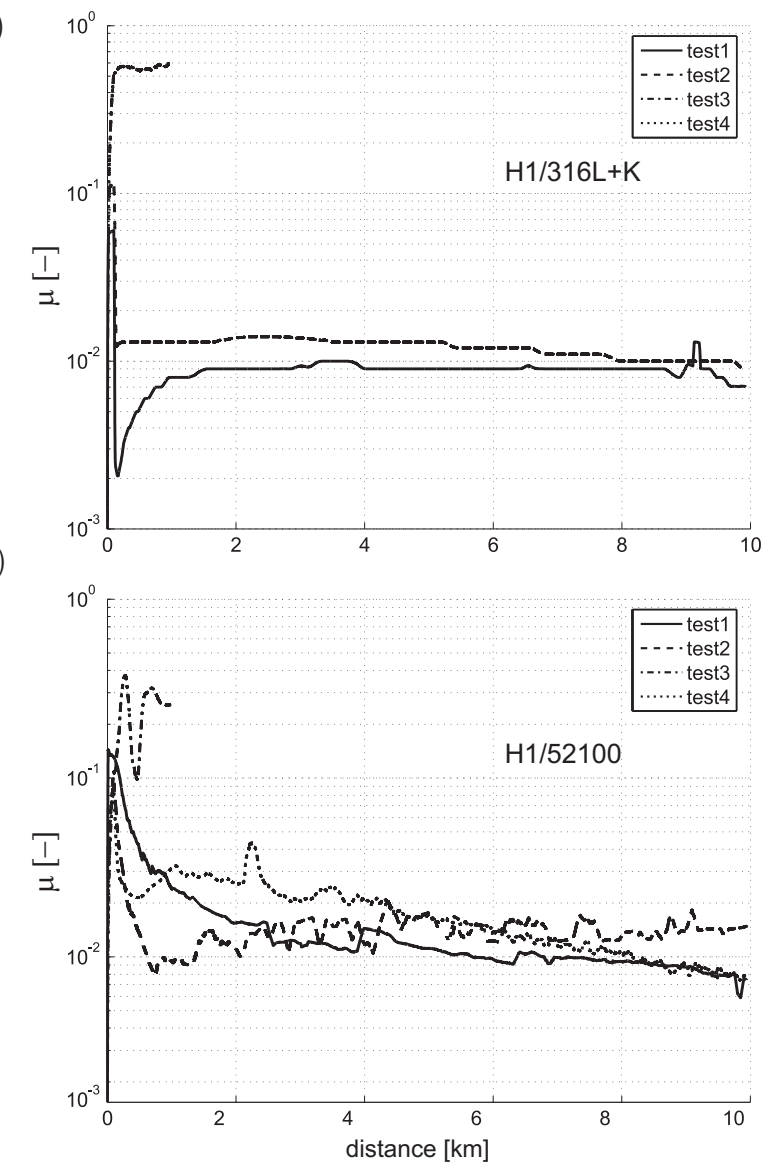

Figure 3.11: CoF of the sliding pairs: a) the coating $\mathrm{H} 1$ on $316 \mathrm{~L}+\mathrm{K}$ steel and b) the H1 coating on 52100 steel both measured in helium

material transfer of the carbonous coating.

Microscopic inspections of the surfaces revealed that all the coatings showing $\mu>0.3$ failed indeed. The averaged $\mu$ values are summarized in table 3.3 .

\section{Long distance measurements}

In the application to piston compressors both long lifetime and low CoF are crucial. Therefore the sliding distance of the following experiments was extended to $25 \mathrm{~km}$ with the intention to assess the coating's sliding durability. The pin samples were coated with the H1 coating, as the counter sliding surface, the 52100 discs were used. The test conditions are given in table 3.6. 


\begin{aligned} & \hline normal load $1 \mathrm{~N} \\ &$ sliding velocity $1 \mathrm{~m} / \mathrm{s} \\ &$ temperature $\mathrm{RT} \\ &$ disc material 52100 \\ & disc surface $\mathrm{Ra}=0.02 \\ &$ atmosphere helium \\ & sliding distance $25 \mathrm{~km} \\ &$\hline\end{aligned}

Table 3.6: Test conditions

Figure 3.12 shows CoF measurements from two experiments, named ld13He and ld14He, performed under the same conditions in helium on the $\mathrm{H} 1$ coatings. Both tests were intended to run for $25 \mathrm{~km}$ sliding distance. However, the test ld14He has been terminated due to problems related to the data logging system. The other two coatings failed at the start of the test. This premature termination allowed us to compare material wear after the sliding distances of 10 and $25 \mathrm{~km}$ under the same conditions.

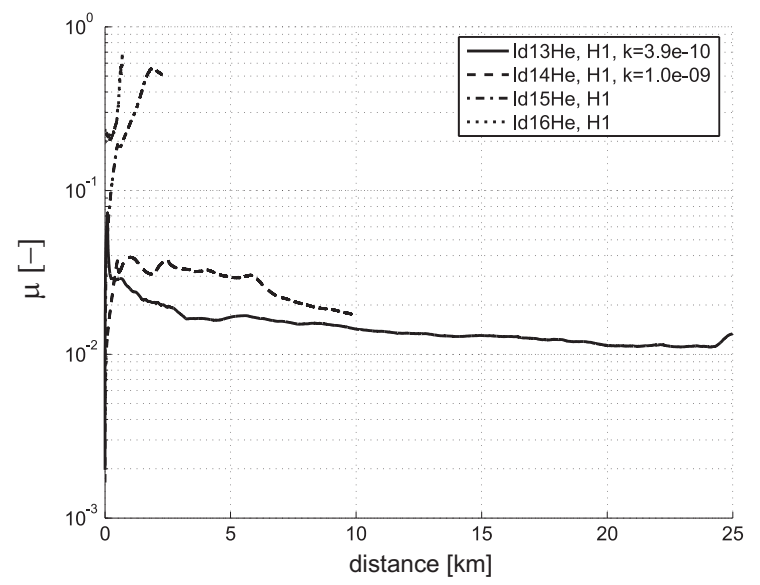

Figure 3.12: CoF of the H1 coatings measured in helium with 52100 discs

The degree of material wear was inspected with a light microscope. Figure 3.13 shows wear pictures after the two experiments ld13He and ld14He. As can be seen the worn spots have nearly the same diameters $D_{l d 13 H e}=355 \mu \mathrm{m}$ and $D_{l d 14 H e}=360 \mu \mathrm{m}$ after the sliding distance of respectively $10 \mathrm{~km}$ and $25 \mathrm{~km}$. It can be concluded that the sliding distance has no effect on the material wear as long as the transfer layer has been formed.

Figures $3.13 \mathrm{~b}$ and 3.13d show the corresponding disc surfaces. All the discs were mirror polished prior to measurements and interference microscope measurements revealed that the surface roughness was low, $\mathrm{Ra}=0.02 \mu \mathrm{m}$. However, as can be seen 
a)

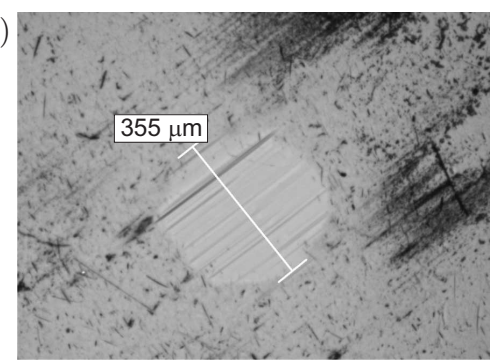

b)

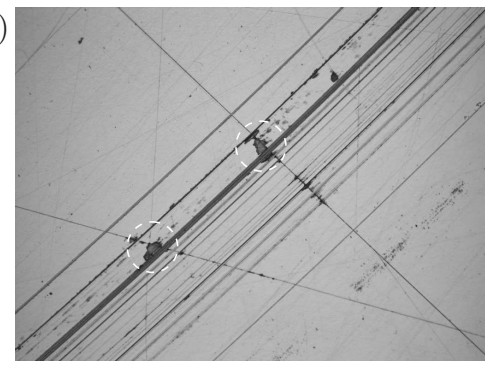

c)

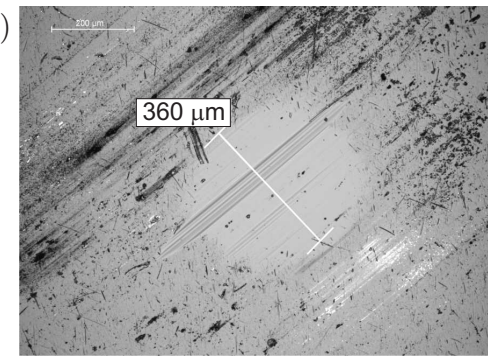

d)

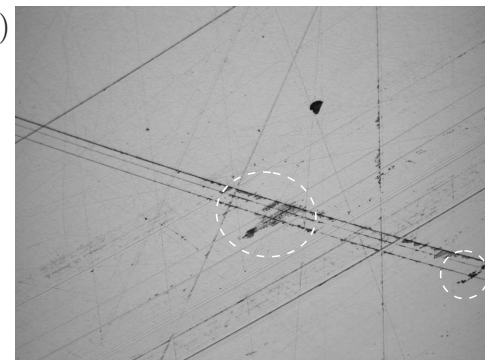

Figure 3.13: Wear observations after the experiments performed on coatings H1: a) the pin worn spot and b) corresponding sliding track on the 52100 disc after the test ld13He c) the pin worn spot and d) sliding track on the 52100 disc after the test ld14He.

on the images there are many random scratches that may result from previous grinding or unproper handling. Comparing to a single asperity scratch, due to the scratching action a groove is formed in the softer surface flanked by two shoulders. The shoulders geometry may have a detrimental effect on the coating wear. The coated pin when passing the grooves would be successfully ground until the grooves are filled with the transferred DLC material. Indeed, as can be observed, a large amount of transferred material is located near the post-grinding groves. This effect may also be responsible for the low reproducibility of the coating's performance.

In Appendix G results of $\mathrm{CoF}$ measurements in vacuum are presented. Wear observations with SEM and EDX spectroscopy measurements were also performed which gives some more insight on the material transfer. In general, we observed more coating failures. This can be explained by the poor convective heat transfer in vacuum.

\subsection{Discussion}

The basic tribological issues regarding dry sliding of coated surfaces in the application to Stirling coolers were discussed. The working environment is characterized mainly 
by low contact pressure, oscillatory temperature, reciprocating motion and inert atmosphere. Various coatings were considered. The DLC coatings have the potential to significantly reduce frictional losses and material wear in sliding mechanical components. The CoF characteristics of the examined coatings are far lower than those for other solid lubricants, in fact far lower than those attained with any other eligible surface-finishing process. The good protection against wear will improve the service lifetime of coated components.

Detailed study has shown that the combination of specific mechanical properties with weak surface interaction forces accounts for the low friction and high wear resistance of DLC coatings. Test conditions and the environment can dramatically influence the tribological performance. The wear and friction behavior of amorphous hydrogenated (a-C:H) DLC coatings have been investigated in a controlled environment using the newly developed vacuum tribotester. As illustrated in this chapter DLC coatings can provide unique properties. The combination of excellent wear resistance and low friction makes these coatings ideal for severe tribological applications under dry sliding conditions in inert gas (helium). The exceptional tribological behavior can be explained by the specific mechanical properties (intrinsic) and favorable environment (extrinsic), see section 3.5.2. The viscoplastic-like properties and structure relaxation of those materials permit weaker interaction between asperities on the sliding surfaces. On the other hand, the hydrogen-terminated carbon surface provides no chemical or physical interactions during sliding. If only one surface is coated the formation of transferred material on the uncoated surface is paramount to achieve low friction. The presence of transferred material on disc surfaces was also observed in those experiments. We can also conclude that if the material transfer occurs the surface wear rate can be exceptionally low. Another advantage is that the worn material is only partially in the form of free wear debris. Most of the worn material is transferred to the counter surface. This has the potential to minimize pollution of the working gas and deterioration of a Stirling cooler performance.

Published experimental results show that DLC surfaces can be protected by a reaction with gases, which could be present in the material or provided on purpose to the working gas. For the application to wear protection and friction reduction in an inert atmosphere, it seems that hydrogen plays a dominant role. Thus, considering sliding surfaces in Stirling compressor, hydrogen included in the working gas may substantially reduce friction and wear.

From the above it follows that DLC coating can be the solid lubrication solution for our design problem. Although, failures of the coatings often occur that are not fully understood, it should be noticed that the contact conditions are more severe on the pin-on-disc configuration then those expected on the piston/cylinder interface (conforming surfaces of low normal force). The contact stresses are considerably higher. In addition, the roughness on both pin and disc surfaces increases contact stresses. Also, the disc surfaces contain some post grinding scratches. This can accelerate wear rate since it can successively cut and remove the coating. Other causes of coatings failure can be related to the type of motion. As for the rotating sliding the available amount of DLC material to be transferred from the pin sliding 
spot to the disc surface is relatively small compared to the disc surface that needs to be covered. The actual conditions on the piston/cylinder interface are expected to be less critical. This is because of lower contact stresses and larger material volume available on the coated surface (piston) compared to the sliding area on the counter surface (cylinder).

\subsection{Conclusions}

In the experimental study four sets of coatings were investigated. The $27 \mathrm{~A}$ coatings sliding against Kolsterised AISI 316L can run-in to a state of ultra-low CoF, $\mu=0.02$ and wear coefficient, $k$ in the order of $10^{-7}$ was achieved. All tests performed on the $26 \mathrm{~A}$ samples ended with premature failure.

The performance of DLC coatings delivered by Hauzer differs when comparing the friction characteristics obtained with different disc materials. The discs made of $316 \mathrm{~L}+\mathrm{K}$ generate a relatively smooth friction signal over the sliding distance. The discs made of 52100 give a more irregular signal with a more pronounced runningin stage. A similar trend can be seen for both types of coating. The difference in the performance is related to the surface chemistry. Namely, the high carbon concentration on the Kolsterized surface may offer favorable conditions for the material transfer of the carbonous coating. In the experiment named test 4 , see figure 3.10a the lowest volumetric wear coefficient was measured, $\mathrm{k}=2.5 \times 10^{-10}$.

The lowest CoF, $\mu=0.0012$ was measured in vacuum during the long distance measurement, named ld8vac, shown in Appendix G. Detailed wear inspections confirmed that the wear debris from the DLC coated pin forms a thick transfer layer on the disc surface. Moreover, wear observations on the coatings H1 after the two experiments named $\mathrm{ld} 13 \mathrm{He}$ and $\mathrm{ld} 14 \mathrm{He}$ revealed that the sliding distance has no effect on the material wear as long as the transfer layer has been formed. In other words, when the transfer material layer is formed the surface wear is terminated.

Some experiments on the coatings (27A, H1, NFC) investigated during this project proved to provide superlow CoF and considerably minimize surface wear. However, reproducibility of those coating's performance is another issue and needs to be improved. From the results discussed above, it can be concluded that the DLC coatings have very promising tribological properties. But, in order to make those coatings competitive to conventionally used coatings in piston compressors their reliability must be higher.

\section{Appendices}

- Appendix D, briefly presents the DLC coatings deposition techniques,

- Appendix E, the environmental effects on sliding properties of DLC coatings,

- Appendix F, describes in detail the vacuum tribo-tester, 
- Appendix G, experimental results on DLC coatings performed in vacuum. 


\title{
FEM-ALE model for
} thermo-mechanical distortion analysis of sliding components

\begin{abstract}
A numerical model will be presented for analysis of heat transfer and thermomechanical distortions in a piston/cylinder assembly (PCA). In a design process of mechanical components Computer Aided Design (CAD) systems assisted with Finite Element Method (FEM) packages are widely accepted as effective tools for design verification. CAD is mainly used for detailed engineering 3D models and $2 \mathrm{D}$ drawings of mechanical components. The output of a CAD model contains information such as dimensions with corresponding tolerances and materials. With FEM stress and deformation analysis of a mechanical entity under defined loading can be performed to investigate the influence of a range of design parameters (like material properties, geometry, sliding conditions, etc). FEM is based on mesh discretization of a continuous domain into a set of discrete sub-domains. When sliding contact of two deformable bodies is to be simulated, the meshes of the bodies need to match across the contact surface. This can be accomplished by application of remeshing or by a renewed contact search. However contact search algorithms and remeshing consume major computational time. As a remedy, the sliding contact problem of two bodies in this thesis is partly modeled as a flow problem. A stationary body is descretized with a Lagrangian mesh and the moving body with an Eulerian mesh. This has the advantage that the meshes on both sides of the contact remain in conformity.
\end{abstract}

The presented FEM model will be used to analyze the distortions of piston/cylinder assembly operating under BL regime, see section 1.2. Gas flow in the piston/cylinder clearance and related effects are not considered here. In order to provide an efficient model for accurate numerical simulations the model must be able to account for the following: 
1. heat transfer on the gas/surface interface

2. heat transfer in the solid body

3. thermal expansion of the solid body

4. contact pressure between two sliding bodies

5. heat transfer between two solid bodies in contact

6. heat generated due to friction on sliding surfaces

First, the different formulations used in the FEM simulations will be compared. The model description starts with the basic equations that governs material deformation and heat transfer. Subsequently the thermo-mechanical contact problem will be discussed. The proposed model is demonstrated on a simple contact problem where a block is sliding on a surface. The obtained numerical results will be validated with the analytical solution of subsurface stress distribution and the energy balance equation.

\subsection{Types of FEM formulations}

The Lagrangian formulation is the most widely used in solid mechanics. In the Lagrangian formulation the state variables (here; stresses, strains, temperature) are functions of the material coordinates $X$. In other words the mesh (also called grid, i.e. reference frame) is fixed to the material point and deforms accordingly. Therefore the material time derivatives of state variables can easily be obtained. In this formulation the reference frame is equal to the initial geometry (Total Lagrangian) or equal to the deformed geometry and moving with the material (Updated Lagrangian). At the end of a time step the referential situation is updated to the current situation, the time derivatives are obtained on the updated mesh. New state variables are calculated after solving the material time increment. Since the mesh is fixed to the material, free surfaces are accurately followed.

The Eulerian formulation is less popular in solid mechanics. Eulerian models are most applied in simulations of very large deformations and fluid flows. Their advantage follows from the fact that Eulerian elements do not deform with the material. Therefore regardless of the magnitude of deformation, Eulerian mesh retains its original shape. Where in Lagrangian formulations the history dependent state variables are readily known, in Eulerian formulations they have to be convected through the mesh every step. Moreover it's often more difficult to describe the boundary conditions accurately. Since free surfaces are never coinciding with the element edges, tracking the free surface is not as straightforward as in Lagrangian formulations.

The Arbitrary Lagrangian Eulerian (ALE) method is a combination of both above mentioned formulations; the mesh displacement can be controlled independently from the material displacement. This method originates from fluid structure interaction 
analysis in the field of fluid dynamics. Later it was introduced for forming processes analysis to avoid mesh distortion when modeling large deformations [48] [47] [82] [83] and to simplify contact description [42] [81]. An ALE calculation step involves two additions with respect to an Updated Lagrangian step. The first is a strategy to reposition the mesh such that a certain mesh quality is maintained. In our case this means maintaining conformity of the meshes across the contact. The second adaptation is a mapping of the state variables to the repositioned mesh from the Lagrangian mesh. In our case this involves solving a transient convection problem.

\subsection{Problem formulation}

Two deformable and thermally conductive bodies in relative motion are considered. Heat loads are applied on external surfaces due to both convective heat transfer and sliding friction. This leads to a thermo-mechanical problem including contact where two fields interact, namely temperature and deformation. The thermo-mechanical problem to be solved is defined by the strong formulations of conservation of energy and momentum. The strong formulations of the problem are to find solutions for the temperature $\mathrm{T}$ and displacements $\mathbf{u}$ within the domain, so that the equilibrium equations and the boundary conditions are fulfilled.
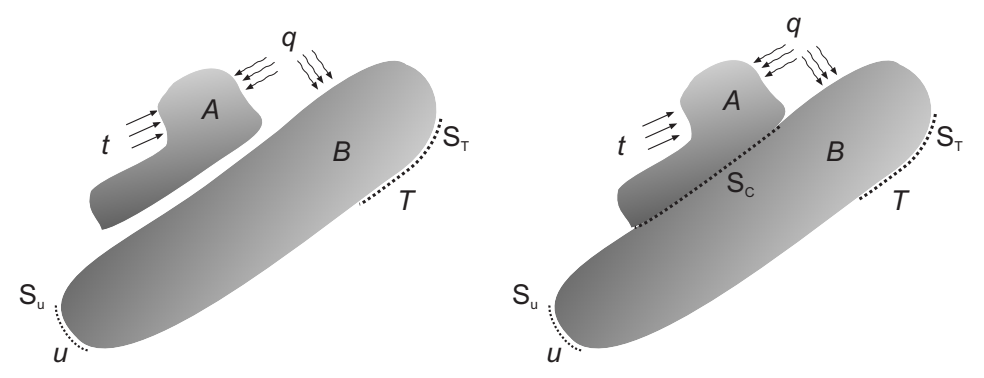

Figure 4.1: Problem definition

The problem is outlined in figure 4.1 together with the boundary conditions. The essential boundary conditions that must be fulfilled are:

$$
\begin{aligned}
& \mathbf{u}=\mathbf{u}_{0} \text { on } S_{u} \\
& T=T_{S} \text { on } S_{T}
\end{aligned}
$$

While the natural boundary conditions are:

$$
\begin{aligned}
& \boldsymbol{\sigma} \cdot \mathbf{n}=\mathbf{t} \quad \text { on } S_{t} \\
& \mathbf{q} \cdot \mathbf{n}=q \quad \text { on } S_{q} \\
& -\mu \boldsymbol{\tau} \cdot \mathbf{n d}=q^{A} \cdot \mathbf{n}+q^{B} \cdot \mathbf{n} \text { on } S_{C} \\
& \boldsymbol{\tau} \cdot \mathbf{n}=\mathbf{t}_{C} \quad \text { on } S_{C}
\end{aligned}
$$


The material behavior is assumed to satisfy generally applied constitutive equations for elastic material including thermal effects. The temperature evolution in a solid is governed by the equation of energy conservation which follows from thermodynamics. The energy conservation in the absence of internal heat sources is written:

$$
\nabla \cdot \mathbf{q}+\boldsymbol{\sigma}: \mathbf{D}-\rho c_{p} \dot{T}=0
$$

where $\mathbf{q}$ is the heat flow, $\rho$ is the mass density, $c_{p}$ is the thermal heat capacity. The effect of mechanical dissipation, the $\boldsymbol{\sigma}: \mathbf{D}$ term is at least three orders lower than the applied external heat. For material thermal equilibrium this term will be neglected. However, for contact thermal equilibrium this term is fundamental.

The equilibrium equation in the absence of inertia and body forces in the current configuration can be written as:

$$
\boldsymbol{\sigma} \cdot \nabla=0
$$

where $\boldsymbol{\sigma}$ is the Cauchy stress. The Cauchy stress is expressed in terms of strain by means of a constitutive law.

The total strain is assumed to consist of the sum of independent elastic and thermal strains,

$$
\epsilon=\epsilon^{e l}+\epsilon^{t h}
$$

The stress is assumed to be proportional to the elastic strain,

$$
\boldsymbol{\sigma}=\mathbf{E}: \boldsymbol{\epsilon}^{e l}
$$

Here $\mathbf{E}$ is the fourth order elasticity tensor $\mathbf{E}=2 G(\mathbf{I}-(1 / 3) \mathbf{1 1})+B \mathbf{1 1}$, where $G$ and $B$ are the shear and bulk modulus. For the stress we find then:

$$
\boldsymbol{\sigma}=\mathbf{E}:\left(\boldsymbol{\epsilon}-\boldsymbol{\epsilon}^{t h}\right)
$$

For isotropic materials the strain due to thermal expansion is:

$$
\boldsymbol{\epsilon}^{t h}=\alpha T \mathbf{1}
$$

where $\alpha$ is the thermal expansion coefficient.

The thermo-elastic constitutive equation can be written with the thermal material tensor defined as $\mathbf{K}=-\alpha B \mathbf{1}$ :

$$
\boldsymbol{\sigma}=\mathbf{E}: \boldsymbol{\epsilon}+\mathbf{K}:\left(T-T_{0}\right)
$$

The contact between the sliding surfaces must be well predicted. As we have to deal with dry sliding surfaces the numerical model must be able to account for friction generated heat and contact heat exchange. The heat generation due to friction, $\mathbf{q}_{f}$, between sliding surfaces is given by the mechanical dissipation rate:

$$
\mathbf{q}_{f}=\boldsymbol{\tau} \cdot \mathbf{d}
$$

where $\boldsymbol{\tau}$ represents vector of contact stresses and $\mathbf{d}$ the relative surface velocity. 
During the solution process of a general contact problem including large relative motion, the active part of the contact interface has to be determined. In the active part of the contact an initial gap vanishes or becomes negative. The contact model should satisfy the Kuhn-Trucker conditions:

$$
\begin{gathered}
g \geq 0 \\
\tau_{3} \leq 0 \\
\tau_{3} \cdot g=0
\end{gathered}
$$

This states that the product of the normal contact stress $\tau_{3}$ and the gap width $g$ is always zero. If there is contact the gap width is zero and the contact stress negative. When the bodies do not make contact the gap width is positive and the contact stresses equal zero.

Friction is modeled in a similar manner as elasto-plastic material behavior. A constitutive relation between the surfaces separation, the gap $g$, the relative surface velocity $\mathbf{d}$ and the contact stress $\boldsymbol{\tau}$ is defined. It is assumed that the normal component of $\mathbf{d}$ is completely elastic and that the tangential component of the relative velocity can be decomposed into a reversible and irreversible part: $\mathbf{d}=\mathbf{d}^{\text {rev }}+\mathbf{d}^{i r}$. This decomposition allows to describe the stick-slip condition. Where the reversible part represents the stick and the irreversible represent the slip condition. The transition from stick to slip is governed by the contact function $\left(\Phi^{C}\right)$. This function divides the contact stress space into a region where stick occurs $\left(\Phi^{C}<0\right)$ and where slip takes place $\Phi^{C}=0[7]$.

In contact mechanic analysis mainly two methods are used; Lagrangian multipliers and the penalty formulation. The former introduces additional variables in the system of equations, but does fulfill the constrains correctly. The latter assumes certain penetration into the contacting bodies, but has no additional variables. The sliding contact here is modeled according to the penalty formulation, so called the contact layer approach [81]. In reality a contact between two surfaces is made via local spots. The height of an asperity is very small compared to the dimension of the total contact area. Hence the contact is modeled as a layer with a very small, finite thickness $\triangle x$. The position of two related points is shown in figure 4.2. Two coordinate systems are defined; global x,y,z, and local x',y',z'. The local coordinate system is defined in the reference surface $S_{r}$ having equal normal distance to the surfaces $S_{A}$ and $S_{B}$ of the contacting bodies $A$ and $B$. This coordinate system is used to account for contact stresses and surface relative velocity $\mathbf{d}$.

a)

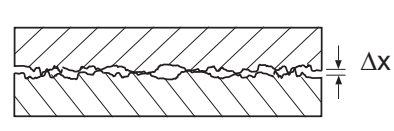

b)

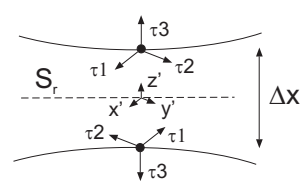

Figure 4.2: a) Contact zone and b) contact layer model 
The contact stress is written as:

$$
\dot{\tau}=\mathbf{E}^{C}:\left(\mathbf{d}-\mathbf{d}^{i r}\right)
$$

The definitions of $\mathbf{d}$ and the rate of contact stress are elaborated in [46] [81] [82]. The diagonal contact stiffness tensor $\mathbf{E}^{C}$ in matrix form is:

$$
\mathbf{E}^{C}=\left[\begin{array}{ccc}
E_{11} & 0 & 0 \\
0 & E_{22} & 0 \\
0 & 0 & E_{33}
\end{array}\right]
$$

The contact stiffness $E_{33}$ in normal direction is the penalty, which prevents the penetration of one body into the other. The tangential stiffness'es $\left(E_{11}\right.$ and $\left.E_{22}\right)$ are equal and depend on the friction coefficient, $\mu$.

In the above section the basic formulations regarding material elastic deformation, heat transfer, and contact were introduced. The objective now is to find the material displacements and temperatures $(\mathbf{u}, \mathrm{T})$ that satisfy the equilibrium equation (4.4) and conservation energy equation (4.3) subjected to the boundary conditions. The formulated problem will be solved according to the ALE procedure. The following sections explains derivation of the FEM equations and the solution procedure according to the UL method. The mechanical and thermal equilibrium will be combined into one system of equations:

$$
\left[\begin{array}{c}
\mathbf{K}^{M M} \mathbf{K}^{M T} \\
\mathbf{K}^{T M} \mathbf{K}^{T T}
\end{array}\right] \cdot\left[\begin{array}{c}
\triangle \mathbf{u} \\
\triangle \mathbf{T}
\end{array}\right]=\left[\begin{array}{c}
\mathbf{F}-\mathbf{R} \\
\mathbf{\Phi}
\end{array}\right]-\left[\begin{array}{c}
\mathbf{0} \\
\mathbf{\Lambda} \mathbf{T}^{\mathbf{n}-\mathbf{1}}
\end{array}\right]
$$

In the decoupled ALE method, each time step is subdivided into two parts which are solved separately [86]. First, UL step is carried out solving for material displacements $u_{m}$ and temperatures $T_{m}$ which correspond to grid displacements $u_{g}$ and temperatures $T_{g}$.

\subsection{Weak equilibrium}

The numerical procedure for solving the problem outlined in section 4.2 consists of solving the weak formulations of equilibrium. The weak formulation can be obtained by weighing the equilibrium equation together with the natural boundary conditions in an integral formulation. In the coupled thermo-mechanical contact problem two fields interact: deformation and temperature. Both need to be considered in the weak formulation.

First we consider the mechanical equilibrium (4.4) weighed with admissible variational velocity fields, $\delta \mathbf{v}$. After applying divergence theorem the weak mechanical equilibrium reads:

$$
\delta W^{M}=\int_{\Omega}(\nabla \delta \mathbf{v}: \boldsymbol{\sigma}) d \Omega-\int_{S_{t}}(\delta \mathbf{v} \cdot \mathbf{t}) d S=0
$$


In the same way as weighing the strong form of mechanical equilibrium the thermal equilibrium can also be weighing with an admissible variation temperature fields, $\delta T$. The thermal equilibrium in the weak form is written as:

$$
\delta W^{T}=\int_{\Omega} \delta T\left(\rho c_{p} T\right) d \Omega-\int_{\Omega}(\delta T \nabla) \cdot \mathbf{q} d \Omega-\int_{S_{q}} \delta T q d S=0
$$

In order to solve the thermo-mechanical problem including contact the weak equations of equilibrium must be extended to two continuous bodies with a common surface. On this surface the penetration condition and a friction law applies.

Considering the contact surface $S_{C}$ only, the weak form of mechanical equilibrium is [82] [81]:

$$
\int_{S_{C}}\left(\delta \mathbf{v}^{A}-\delta \mathbf{v}^{B}\right) \cdot \boldsymbol{\tau} d S=0
$$

where the condition (4.11) must be satisfied. The variational velocity fields $\delta \mathbf{v}^{A}$ and $\delta \mathbf{v}^{B}$ belong to the surfaces in contact of body $A$ and $B$ respectively. Adding (4.17) to the weak form for a single body (4.15), the weak mechanical form for two contacting bodies can be written as:

$$
\delta W^{M_{C}}=\int_{\Omega^{A}, \Omega^{B}}(\delta \mathbf{v} \nabla): \boldsymbol{\sigma} d \Omega-\int_{S_{C}}\left(\delta \mathbf{v}^{A}-\delta \mathbf{v}^{A}\right) \cdot \boldsymbol{\tau} d S \int_{S_{t}}(\delta \mathbf{v} \cdot \mathbf{t}) d S=0
$$

Conservation of energy (4.3) over the contact surface reduces by (4.16) to:

$$
\int_{S_{C}} \delta \dot{T}(\boldsymbol{\tau} \cdot \mathbf{d}) d S+\int_{S_{C}}\left(\delta \dot{T}^{A}-\delta \dot{T}^{B}\right) q d S=0
$$

where the scalar heat flow $q$ is normal to the surface. In this weak contact equilibrium $\mathbf{d}=\mathbf{v}^{A}-\mathbf{v}^{B}$ is the relative contact velocity. Joint weighting of both bodies (4.16) and the contact surface (4.19) gives:

$$
\begin{aligned}
\delta W^{T_{C}} & =\int_{\Omega^{A}, \Omega^{B}} \delta \dot{T} \rho c \dot{T} d \Omega-\int_{\Omega^{A}, \Omega^{B}}(\delta \dot{T} \nabla) \cdot \mathbf{q} d \Omega- \\
& \int_{S_{C}} \delta \dot{T}(\boldsymbol{\tau} \cdot \mathbf{d}) d S-\int_{S_{C}}\left(\delta \dot{T}^{A}-\delta \dot{T}^{B}\right) q d S-\int_{S_{q}} \delta \dot{T} h d S=0
\end{aligned}
$$

\subsection{FEM discretization}

The approximate solution for the previously formulated weak equilibrium equations for the thermo-mechanical problem including contact will be evaluated by means of FEM. The discussed model is implemented in the proprietary code DiekA [46] [86]. The two bodies with sliding contact are divided into finite elements. In this work isoparametric bilinear quadrilateral, antisymmetric elements are used. For 
isoparametric elements the same interpolation is applied for both the geometry and the independent field variables [11]. The primary field variables: displacements, temperature (or their increments) are calculated in the element nodes. The state variables inside an element are fully determined by the interpolation functions which depend on the nodal point values and local coordinates.

\subsubsection{Interpolation functions}

The interpolation functions $N$ are $C^{o}$ continuous across element edges and have the value of one on the nodal points.

$$
\begin{aligned}
& \mathbf{x}=\sum N^{\alpha} \mathbf{x}^{\alpha} \\
& \xi=\sum N^{\alpha} \xi^{\alpha}
\end{aligned}
$$

where $N^{\alpha}$ is the time independent linear interpolation function for the node $\alpha$. Integrals over the element domain are determined using numerical integration. Accurate higher order integration is obtained by Gaussian quadrature, [1].

\subsubsection{Thermal discretization}

Starting from the weak form of thermal equilibrium (4.16) all continuous field variables are described by the proposed interpolation functions. For the nodal degrees of freedom the interpolations are written;

$$
\begin{array}{cc}
\mathbf{v}=\sum_{\alpha} N^{\alpha} \mathbf{v}^{\alpha} & \delta \mathbf{v}=\sum_{\alpha} N^{\alpha} \delta \mathbf{v}^{\alpha} \\
\dot{T}=\sum_{\alpha} N^{\alpha} \dot{T}^{\alpha} & \delta \dot{T}=\sum_{\alpha} N^{\alpha} \delta \dot{T}^{\alpha}
\end{array}
$$

The rate of deformation is written now in index notation, as:

$$
D_{i, j}=\frac{1}{2}\left(v_{i, j}+v_{j, i}\right)=\frac{1}{2} \sum_{\alpha k}\left(N_{, j}^{\alpha} \delta_{i k}+N_{, i}^{\alpha} \delta_{j k}\right) v_{k}^{\alpha}=\sum_{\alpha k} B_{i j k}^{\alpha} v_{k}^{\alpha}
$$

and in the tensor form

$$
\mathbf{D}=\mathbf{B} \cdot \mathbf{v} \quad \delta \mathbf{D}=\mathbf{B} \cdot \delta \mathbf{v}
$$

As for the material rate of deformation, the relative contact velocity can be written to yield:

$$
\mathbf{d}=\mathbf{B}_{C}^{\alpha} \cdot \mathbf{v}^{\alpha} \quad \delta \mathbf{d}=\mathbf{B}_{C}^{\alpha} \cdot \delta \mathbf{v}^{\alpha}
$$

where $\mathbf{B}_{C}^{\alpha}$ is a second order tensor that relates the relative surface velocity vector to the nodal velocities. The temperature difference between opposite nodes in the 
contact $\triangle T_{C}=T^{A}-T^{B}$ is evaluated by projection onto the local normal direction. The heat flow perpendicular to the contact surface can be written as:

$$
q=\kappa \triangle T_{C}
$$

where $\kappa$ is the contact heat transfer coefficient.

The unknown field variable heat flux in equation (4.16) can be expressed in an other field variable, temperature and therefore be eliminated from the solution procedure. Considering the Fourier law, for small increments it can be written:

$$
\mathbf{q}+\triangle \mathbf{q}=-\lambda \nabla(T+\triangle T)
$$

where $\lambda$ is the (isotropic, thus a scalar) thermal conductivity. This implies that:

$$
\triangle \mathbf{q}=-\lambda \nabla \triangle T
$$

Substituting the Fourier law in the equation of energy conservation, written in the rate incremental form yields:

$$
\rho c_{p} \triangle T=\nabla \lambda \nabla(T+\theta \triangle T) \triangle t
$$

An implicit scheme is applied to the conductivity, the optimal value of $\theta=2 / 3$ is recommended [89]

Since the problem will be solved in the rate form the equation is split into a diffusion term acting on the material rate of change of the temperature and a term acting on the initial temperature:

$$
\delta W^{T}=\int_{\Omega}(\delta T \rho c \triangle T+\theta \lambda \triangle t \nabla \delta T \cdot \nabla \triangle T) d \Omega=-\lambda \triangle t \int_{\Omega} \nabla \delta T \nabla T d \Omega
$$

With all the interpolations defined, the weak formulation of thermal equilibrium for a situation of contacting bodies $A$ and $B$ can be rewritten to yield the finite element discretization:

$$
\begin{gathered}
\delta W^{T_{C}}=\int_{\Omega^{A}, \Omega^{B}} \delta T(\rho c \triangle T+\theta \lambda \triangle t \nabla \delta T \cdot \nabla \triangle T) d \Omega+\lambda \triangle t \int_{\Omega^{A}, \Omega^{B}} \nabla \delta T \nabla T d \Omega \\
\int_{S_{C}} \delta T \kappa \triangle T^{C} \triangle t d S-\int_{S_{C}} \delta T N \boldsymbol{\tau} \cdot \mathbf{B}_{C} \cdot \mathbf{v} \triangle t d S+\int_{S_{q}} \delta T N q \triangle t d S=0
\end{gathered}
$$

Under the assumption that the equation (4.32) has to hold for all possible temperature variation fields, the discrete system can be written as:

$$
\left[\mathbf{K}^{T T}\right] \triangle T+\left[\mathbf{K}^{T M}\right] \cdot \triangle \mathbf{u}=\left[\boldsymbol{\Phi}^{T}\right]-[\boldsymbol{\Lambda}] \mathbf{T}^{(n-1)}=0
$$

in this equation $\mathbf{K}^{T M}$ denotes dissipative heating on the contact surface and $\mathbf{K}^{T T}$ 
contains the heat capacity and the heat flow due to temperature increment. The $\boldsymbol{\Phi}$ component contains external heat flow.

$$
\begin{aligned}
{\left[\mathbf{K}^{T T}\right] } & =\rho c \int_{\Omega^{A}, \Omega^{B}}\left(N^{T} N+\theta \frac{\lambda \triangle t}{\rho c_{p}} \nabla \delta T \cdot \nabla\right) d \Omega \\
{\left[\mathbf{K}^{T M}\right] } & =\int_{S_{C}} N \tau \cdot \mathbf{B}_{C} \cdot \mathbf{v} \triangle t d \Omega \\
{[\boldsymbol{\Phi}] } & =\int_{S_{q}} N q \triangle t d S+\int_{S_{C}} \kappa \triangle T^{C} \triangle t d S \\
{[\mathbf{\Lambda}] } & =\lambda \int_{\Omega^{A}, \Omega^{B}}(\nabla N)^{T} \cdot(\nabla N) d \Omega
\end{aligned}
$$

\subsubsection{Mechanical discretization}

Upon using the interpolations (4.22) and substituting the constitutive behavior (4.9) the weak form of the mechanical equilibrium of equation (4.15) excluding contact is written as:

$$
\delta W^{M}=\int_{\Omega} \delta \mathbf{v}(\mathbf{B})^{T}:\{\mathbf{E}: \mathbf{B v}+\mathbf{K} N \dot{T}\} d \Omega-\int_{S_{t}} \delta \mathbf{v} N \cdot \mathbf{t} d S=0
$$

Interpolation for the contact elements is defined taking, $\left(\delta \mathbf{v}^{A}-\delta \mathbf{v}^{B}\right)=\delta \triangle \mathbf{v}$ gives

$$
\begin{array}{r}
\Delta \mathbf{v}=\mathbf{N}_{C} \cdot \mathbf{v} \\
\delta \triangle \mathbf{v}=\mathbf{N}_{C} \cdot \delta \mathbf{v}
\end{array}
$$

where $\mathbf{N}_{C}$ is a secondary order tensor. The relation for the contact stress is written in incremental form:

$$
\boldsymbol{\tau}=\boldsymbol{\tau}^{n-1}+\triangle \boldsymbol{\tau}=\boldsymbol{\tau}^{n-1}+\mathbf{L}_{C} \cdot \mathbf{d} \triangle t=\boldsymbol{\tau}^{n-1}+\mathbf{L}_{C} \cdot \mathbf{B}_{C} \cdot \mathbf{v} \triangle t
$$

here $\mathbf{L}_{C}$ contains the contact and stick slip behavior. Mechanical contact behavior is considered independent from temperature [71].

Upon using the interpolations (4.22) the weak form of the mechanical equilibrium of equation (4.15) including contact is written as:

$$
\begin{aligned}
\delta W^{M_{C}} & =\int_{\Omega^{A}, \Omega^{B}} \delta \mathbf{v B}^{T}: \mathbf{E}: \mathbf{B v} \triangle t d \Omega+\int_{\Omega^{A}, \Omega^{B}} \delta \mathbf{v K N} \triangle T d \Omega+ \\
& \int_{S_{C}} \delta \mathbf{v} \mathbf{N}_{C}^{T} \cdot \mathbf{L}_{C} \cdot \mathbf{B}_{C} \cdot \mathbf{v} \triangle t d S+\int_{S_{C}} \delta \mathbf{v} \mathbf{N}_{C}^{T} \cdot \boldsymbol{\tau}^{n-1} d S-\int_{S_{t}} \delta \mathbf{v} N \cdot \mathbf{t} d S=0
\end{aligned}
$$

Collecting the terms containing the independent degrees of freedom on the left side and the constant terms on the right it can be written

$$
\delta W^{M_{C}}=\left[\mathbf{K}^{M M}\right] \cdot \triangle \mathbf{u}+\left[\mathbf{K}^{M T}\right] \triangle \mathbf{T}=[\mathbf{F}]
$$


where $\mathbf{K}^{M M}$ and $\mathbf{K}^{M T}$ contains mechanical behavior and thermal expansion respectively and $\mathbf{F}$ denotes the mechanical force.

$$
\begin{aligned}
{\left[\mathbf{K}^{M M}\right] } & =\int_{\Omega^{A}, \Omega^{B}} \mathbf{B}^{T}: \mathbf{E}: \mathbf{B} d \Omega+\int_{S_{C}} \mathbf{N}_{C}^{T} \cdot \mathbf{L}_{C} \cdot \mathbf{B}_{C} \cdot d S \\
{\left[\mathbf{K}^{M T}\right] } & =\int_{\Omega^{A}, \Omega^{B}} \mathbf{B}^{T} \mathbf{K} N d \Omega \\
{[\mathbf{F}] } & =\int_{S_{t}} N \cdot \mathbf{t} d S+\int_{S_{C}} \mathbf{N}_{C}^{T} \cdot \tau^{n-1} d S
\end{aligned}
$$

The mechanical and thermal equilibrium, equations (4.42) and (4.32), can be combined into one system of equations:

$$
\left[\begin{array}{c}
\mathbf{K}^{M M} \mathbf{K}^{M T} \\
\mathbf{K}^{T M} \mathbf{K}^{T T}
\end{array}\right] \cdot\left[\begin{array}{c}
\triangle \mathbf{u} \\
\triangle \mathbf{T}
\end{array}\right]=\left[\begin{array}{c}
\mathbf{F}-\mathbf{R} \\
\mathbf{\Phi}
\end{array}\right]-\left[\begin{array}{c}
\mathbf{0} \\
\mathbf{\Lambda} \mathbf{T}^{\mathbf{n}-1}
\end{array}\right]
$$

It can be seen that the mechanical and the thermal problem are coupled: temperature changes cause isotropic stresses and mechanical work causes temperature increases.

The weak equilibrium equations (4.18) and (4.20) are evaluated at a number of discrete points. At the end of a time increment $t^{n}$ the unknown state and field variables are evaluated based on the previous solution at $t^{n-1}$. In order to obtain global equilibrium at the end of a time increment an iterative algorithm is employed as the solution method for the nonlinear semi discrete equations. Non-linearity in the formulated problem follows mainly from the contact formulation, equations (4.11). This necessitates an incremental solving procedure. In the end, the material displacements and temperature increments $\Delta u^{\alpha}, \Delta T^{\alpha}$ at the nodal point $(\alpha)$ are calculated. This completes the first step of the ALE procedure.

\subsubsection{ALE formulation}

A flow chart of the uncoupled ALE method is shown in figure 4.3. Deviation from the UL procedure occurs in the way the mesh is updated and the new state variables are calculated after solving the material increments. In the ALE formulation the material displacements are uncoupled from the nodal (grid) point displacements. In addition to the incremental (temperature) calculations as in UL, convection must be taken into account in order to be able to update the material state at the grid points.

The displacements at a grid point after a time increment $\Delta t$ is obtained from the material displacements at the previous step and by taking only the radial component:

$$
\mathbf{u}_{g}^{n}=\mathbf{u}_{g}^{n-1}+\triangle \mathbf{u}_{m} \cdot \mathbf{e}_{r} \otimes \mathbf{e}_{r}
$$

The temperature at a grid point after a time increment $\Delta t$ is approximated using an incremental form:

$$
T_{g}^{n}=T_{g}^{n-1}+\triangle T_{m}+\left(\Delta \mathbf{u}_{g}-\Delta \mathbf{u}_{m}\right) \cdot \nabla T_{g}^{n-1}
$$




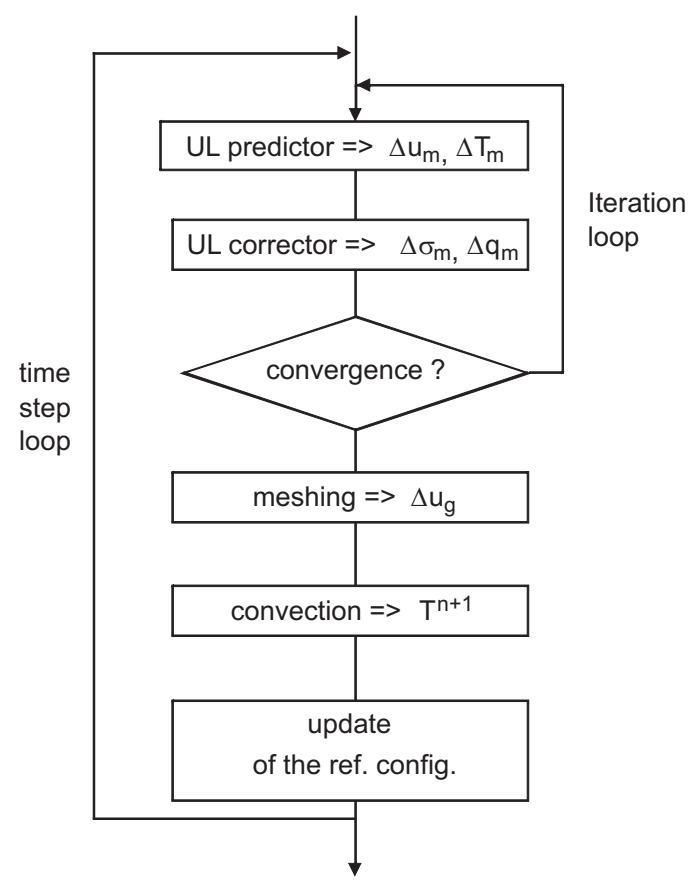

Figure 4.3: Flow chart of the uncoupled ALE method

The second term on the right-hand side of equation (4.49) equals the temperature increment obtained from the UL method, the third term represents the convective temperature increment.

The ALE procedure involves solving the material data at new locations after the grid is repositioned. This means the temperature and displacement fields may not satisfy the equilibrium equations any more. Therefore the obtained residual (equation (4.50)) is taken into account in a subsequent iteration. This is done by solving the equilibrium equations (4.47) again with the residuals on the right-hand side of the equation.

$$
\mathbf{R}=\left[\mathbf{K}^{M M}\right] \cdot \mathbf{u}_{g}+\left[\mathbf{K}^{M T}\right] T_{g}
$$

\subsubsection{Modeling contact}

In the simulations performed in this work, the contact model is combined with ALE formulation. The nodes of the contact elements are displaced convectively in accordance with the nodes on the moving body surface. Consequently convection should be taken into account. The convection in the contact elements is neglected however. This appears not to influence the results of the simulations too much [86]. 


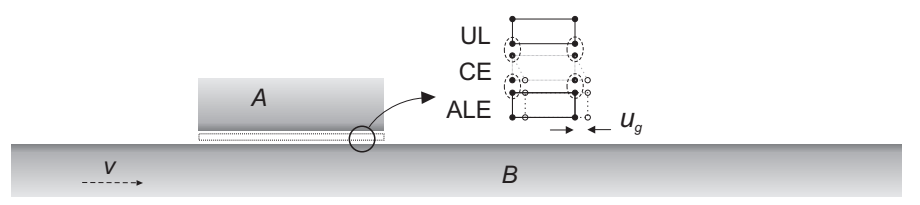

Figure 4.4: Schematic representation of contact modeled with ALE method

Figure 4.4 shows schematically contact modeled between two sliding bodies. Nodes of the contact elements (CE) are connected to the solid elements as depicted. The contact elements are simple node to node elements based on a penalty formulation. They can be seen as springs, having a certain stiffness and thermal conduction, and will be activated when the element thickness becomes zero. Coulomb friction is used with a constant coefficient of friction, $\mu$.

\subsection{Validation}

The discussed model is used to calculate the temperature field in the body $B$ due to the sliding body $A$. Only body $B$ is a heat conductor $\lambda=30 \mathrm{~W} / \mathrm{m}^{2} \mathrm{~K}$. The material data are chosen as for AISI-52100. The lower body $B$ moves with a constant velocity, $v=0.1 \mathrm{~m} / \mathrm{s}$ as depicted, see figure $4.5 \mathrm{a}$. The normal load $F=40 \mathrm{~N}$ results in the contact pressure $\mathrm{p}=4 \mathrm{kPa}$ (contact length, $L=0.01 \mathrm{~m}$ ).

a)

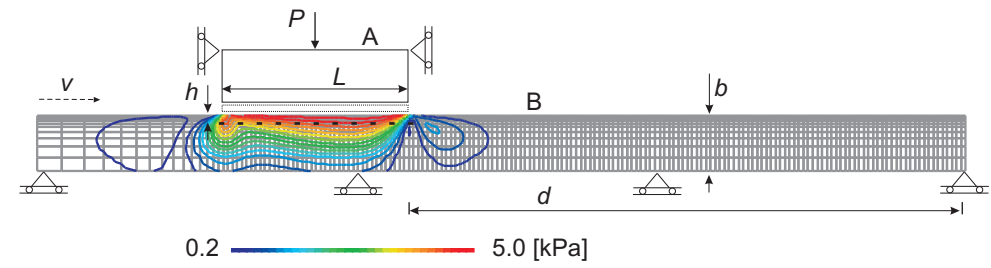

b)

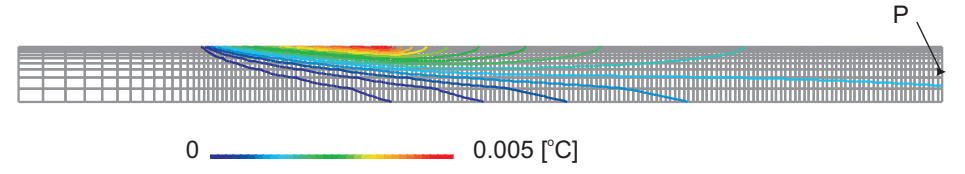

Figure 4.5: Sliding block on plate problem; a) boundary conditions and shear stress distribution, b) temperature field after the time $\mathrm{t}=1.2 \mathrm{sec}$

For validation the material outflow temperature at the point ' $\mathrm{P}$ ' is monitored. The temperature history is shown in the figure 4.6a. Here we compare the material outflow energy to the friction induced heat power. This gives us the following relation;

$$
\Delta T=\sigma_{s h} L /\left(\rho c_{p} b\right)
$$


a)

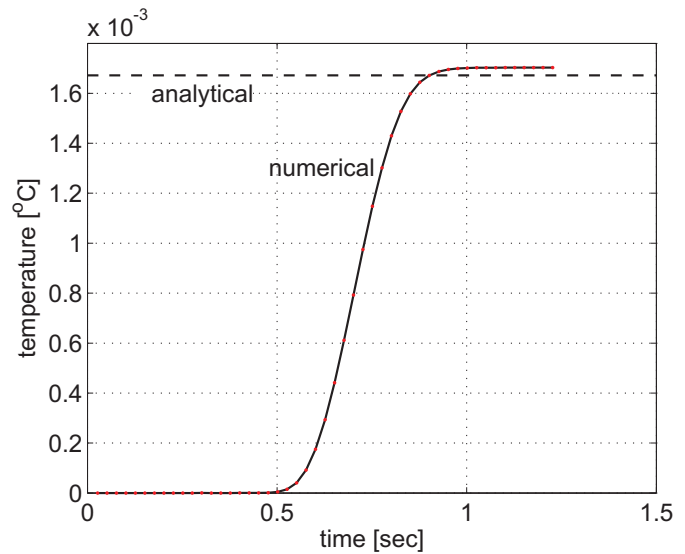

b)

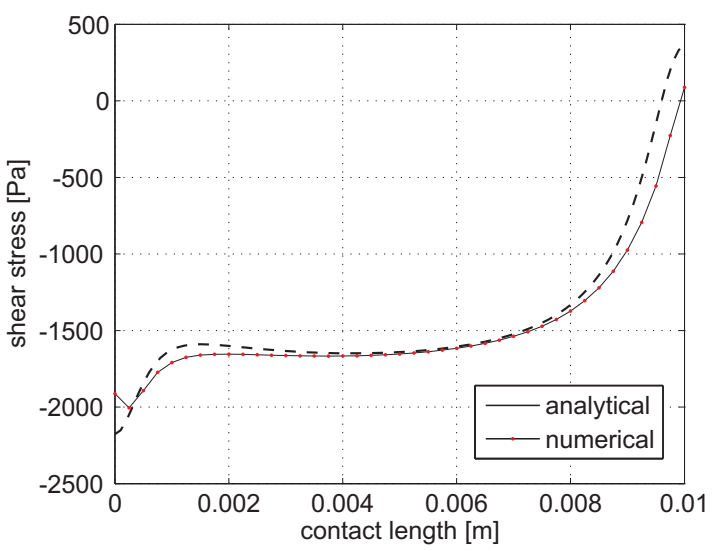

Figure 4.6: Simulation results; a) material temperature at the point ' $\mathrm{P}$ ' also the analytical solution is depicted, b) the shear stress at $0.7 \mathrm{~mm}$ beneath the sliding surface

where $\sigma_{s h}$ shear stress in the contact, $\rho$ material density, $c_{p}$ heat capacity, $d$ and $b$ dimensions. For our case the average material temperature should increase $\Delta T=$ $1.66 \times 10^{-3}{ }^{\circ} \mathrm{C}$. The distance $d$ is chosen sufficient for the temperature to equalize across the width. Attention is also paid to an accurate subsurface stress distribution. Coefficient of friction, $\mu=0.3$ was assumed.

In the figure $4.6 \mathrm{~b}$ the shear stress beneath the surface $(\mathrm{h}=0.7 \mathrm{~mm})$ obtained from the simulations is compared to the analytical solution [49]. We observe good agreement between the two solutions. The inaccuracy on the external sides of the contact can be explained by the coarse mesh. [68] 


\subsection{Concluding remarks}

An alternative numerical model was presented for the analysis of a thermo-mechanical problem including sliding contact with frictional heat generation. One test case was used to validate accuracy of the model. The example of a sliding block on a plate shows that the numerical results are in good agreement with the exact ones considering thermal balance. Subsurface shear stresses were also compared with an analytical solution. The presented method can be useful in simulations of thermo-mechanical distortions in mechanical assemblies. It has the advantage over other FEM that the contact search algorithms are excluded. 



\title{
Analysis of distortions of piston/cylinder assembly
}

\begin{abstract}
The presented numerical model in the previous chapter will be applied to simulate heat transfer between the reciprocating piston and the cylinder liner. For given operating conditions the resulting shape deformation will be estimated. The transient conditions during start-up leading to thermo-mechanical instabilities will be identified for various piston designs and materials. In Chapter 2 different piston designs were considered for improved gas sealing. Based on that discussion, the two configurations, namely; the clearance seal with a classical piston design and the U-piston will be examined.
\end{abstract}

\subsection{The analyzed system}

In the analysis of PCA thermo-mechanical distortions an accurate prediction of the sliding components temperature fields that are responsible for their deformation is crucial. Material deformation due to the thermal expansion is considered as dominant. Based on the results obtained from simulations applying both temperature and pressure on the boundary we concluded that the deformation due to gas pressure can be neglected. The operating conditions were discussed in Chapter 2. It is assumed that the piston is positioned concentric within the cylinder and it remains concentric during operation. This allows us to use axisymmetric elements. The system and its finite element discretization are shown in the figure 5.1a. As depicted the element size is refined towards the surfaces exposed to the heat source to improve accuracy. Element refinement in the cylinder longitudinal direction is limited because of the Courant number restriction [89] ${ }^{1}$.

\footnotetext{
${ }^{1}$ The number says basically that the maximum material displacement may not be larger then the element length in the flow direction within one increment.
} 
Based on the discussion in Chapter 4, the relative motion between piston and cylinder is partly modeled as a flow problem where the stationary body is descretized with a Lagrangian mesh and the moving body with an Eulerian mesh. This has the advantage that the meshes on both sides of the contact remain in conformity which means that no contact search algorithm is needed. The relative motion between the assembly parts is reversed in this analysis as compared to a real piston compressor. The piston is modeled stationary (Lagrangian mesh) and the cylinder material performs an oscillatory motion (Eulerian mesh), see section 4.1. The displacements were prescribed in the flow direction at the bottom cylinder edge as depicted in figure 5.1a. The contact elements are depicted in 5.1b and c. Figure 5.1d shows gas temperature and cylinder displacement variations with time. The convective heat
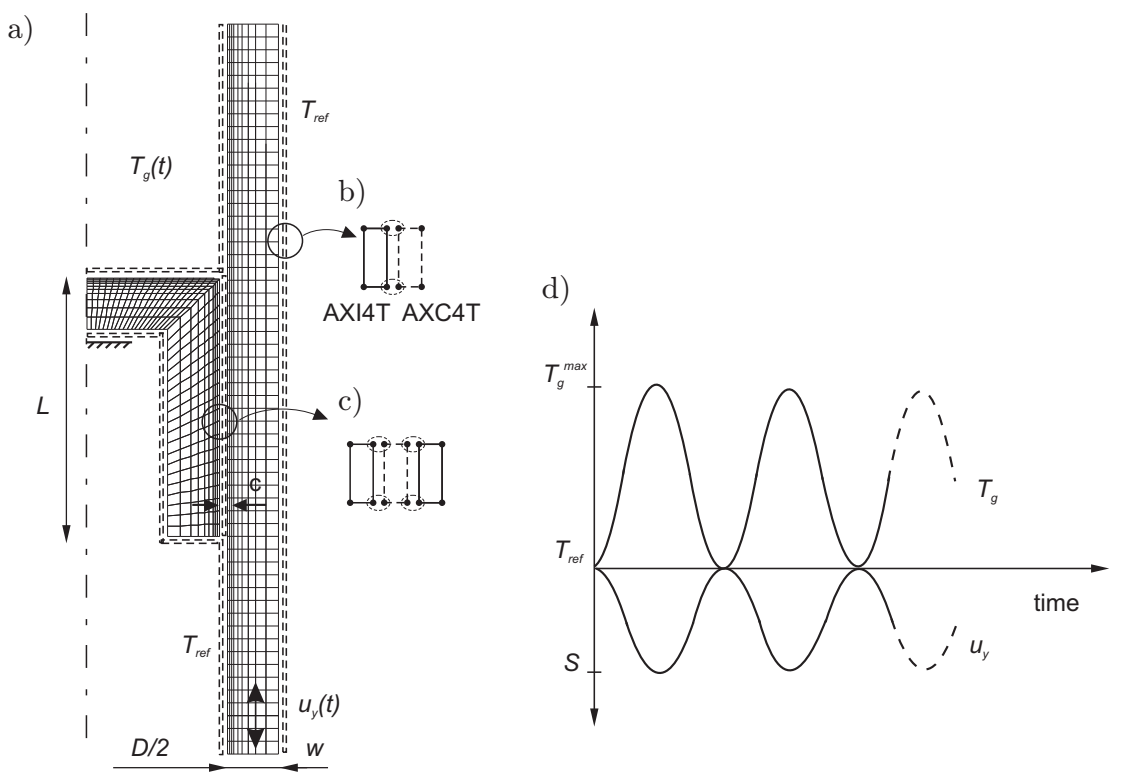

Figure 5.1: The FE model for thermo-mechanical distortion analysis of PCA

transfer between gas and solid is modeled with thermal contact elements, depicted by the dotted lines. The gas temperature is prescribed on the free side and it oscillates between the reference temperature $T_{\text {ref }}$ and $T_{\text {gmax }}$. Heat transfer coefficients for the following surfaces: gas-solid, coolant-solid (the external cylinder surface) and coolantsolid (beneath the piston) are assumed constant in time and space, respectively 500 , 100 and $350 \mathrm{~W} / \mathrm{m}^{2} \mathrm{~K}$ [58]. Baseline data and material properties used for the analysis are summarized in tables 5.1 and 5.2. 


\begin{tabular}{lcl}
\hline piston stroke, $S$ & 0.052 & {$[\mathrm{~m}]$} \\
reference temperature, $T_{r e f}$ & 0 & {$\left[{ }^{\circ} \mathrm{C}\right]$} \\
gas temperature range, $T_{g}$ & $0 . .160$ & {$\left[{ }^{\circ} \mathrm{C}\right]$} \\
heat transfer coefficient, $h_{g / s}$ & 500 & {$\left[\mathrm{~W} / \mathrm{m}^{2} \mathrm{~K}\right]$} \\
heat transfer coefficient, $h_{l c / s}$ & 100 & {$\left[\mathrm{~W} / \mathrm{m}^{2} \mathrm{~K}\right]$} \\
heat transfer coefficient, $h_{p c / s}$ & 350 & {$\left[\mathrm{~W} / \mathrm{m}^{2} \mathrm{~K}\right]$} \\
coefficient of friction, $\mu$ & 0.3 & \\
\hline
\end{tabular}

Table 5.1: Baseline thermal data used in the analysis

\begin{tabular}{lcl}
\hline & 213 & {$[\mathrm{GPa}]$} \\
elastic modulus, $E$ & 0.29 & {$[-]$} \\
Poisson ratio, $\nu$ & $12 \times 10^{-6}$ & {$[1 / \mathrm{K}]$} \\
thermal expansion coefficient, $\alpha$ & 46.6 & {$\left[\mathrm{~W} / \mathrm{m}^{2} \mathrm{~K}\right]$} \\
thermal conductivity, $\lambda$ & 7875 & {$\left[\mathrm{~kg} / \mathrm{m}^{3}\right]$} \\
density, $\rho$ & 300 & {$[\mathrm{~J} / \mathrm{kg} \mathrm{K}]$} \\
specific heat, $c_{p}$ & \\
\hline
\end{tabular}

Table 5.2: Baseline material properties (AISI 52100) used in the analysis

\subsection{Transient analysis}

The classical piston design 5.1 is analyzed under transient BC's. The simulation starts with an initial piston cylinder clearance $\mathrm{c}=3 \mu \mathrm{m}$. Figure $5.2 \mathrm{a}$ shows the temperature field after 8 cycles. The material deformation is exaggerated $(\times 500)$ in order to illustrate the deformed piston and cylinder shapes. Due to the material thermal expansion the upper piston corner touches the cylinder surface first. At this moment the temperature starts increasing rapidly leading to thermo-mechanical instability. Namely, the generated frictional heat involves an increase of material temperature. The temperature is mainly accumulated in the piston head. This is due to the fact that the piston corner is constantly exposed to the heat source during sliding, unlike the cylinder surface where the heat is distributed along the cylinder. The final temperature distribution and the parts deformation are shown in figure $5.2 \mathrm{c}$.

Various calculations were performed to simulate the entire process, from cold start till the steady state. This requires a large number of cycles and subsequently, time steps. From our experience it follows that the Courant number mentioned before must be reduced by a factor of 0.5. This means that the displacement within one time increment must be lower then half of the element length. Assuming operating frequency only $0.83 \mathrm{~Hz}, \Delta t<0.0012$. This means one full cycle needs 1000 steps.

The simulation results with different initial clearance and coefficients of friction are shown in figure 5.3. Two CoF are selected; 0.3 which can be representative for sliding condition of polymer coating and 0.02 which can be representative for sliding condition of DLC coating. It is clear from the plots that the piston sliding with $\mu=0.3$ generates a substantial amount of heat. The piston temperature and thermal expansion increase 
a)

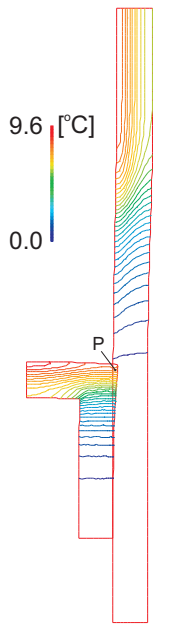

b)

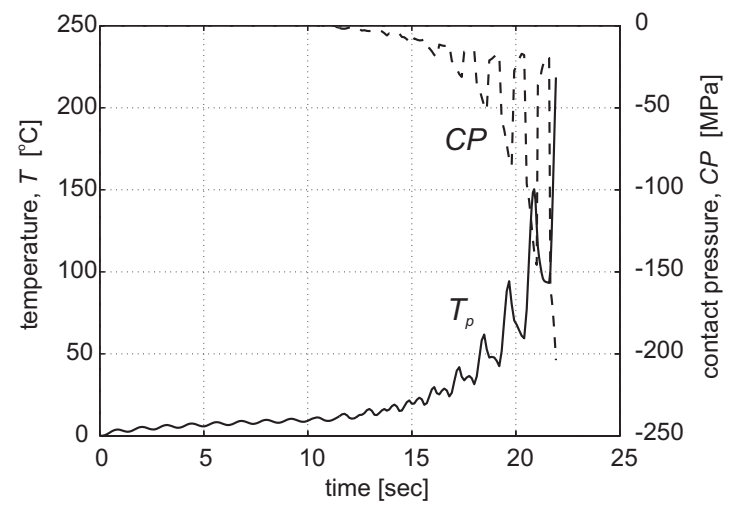

c)

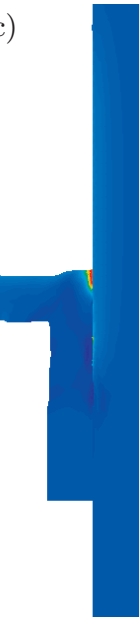

Figure 5.2: Simulation results from transient analysis a) temperature fields after 8 cycles and the deformed contours, b) evolution of piston temperature and contact pressure leading to thermo-mechanical instability, c) deformed shape and temperature field after thermo-mechanical instability

rapidly leading to thermo-mechanical instability. This effect generates high contact stresses. The results with initial clearance of $16 \mu \mathrm{m}$ show temperature increase that saturates to the steady state temperature of $49{ }^{\circ} \mathrm{C}$. As for the results with lower CoF, 0.02 , looking at the figure $5.3 \mathrm{a}$ only the piston temperature evolution with $c=1 \mu \mathrm{m}$ deviates from the main temperature plot. The temperature seems not to be saturating but increasing, nearly linearly. This is caused by the high contact stresses which also increase nearly linearly. The other temperature plots for $c=3,5$ and $16 \mu \mathrm{m}$ follow the same temperature course. Figure $5.3 \mathrm{~b}$ shows clearly how the radial displacement evolves until the moment when contact is established. After that moment the radial displacement is restricted by the cylinder wall. The contact pressure shown in figure $5.3 \mathrm{c}$ for initial clearance larger than $1 \mu \mathrm{m}$ and $\mu=0.02$ have a tendency to saturate. It appears that under those conditions and after ample time a steady state can be reached but with high contact stresses.

\subsection{Alternative piston design}

The assumed advantages of the U-piston were discussed more in Chapter 2. In this section the thermo-mechanical distortions and the ability to seal compressed gas will be discussed. Figure 5.4 shows analytical solution of the contact pressure on the ring/cylinder assembly. The increasing inner diameter of the ring reduces the ring's stiffness and consequently the contact pressure. If the groove length, see $2.6 \mathrm{c}$ is 
a)

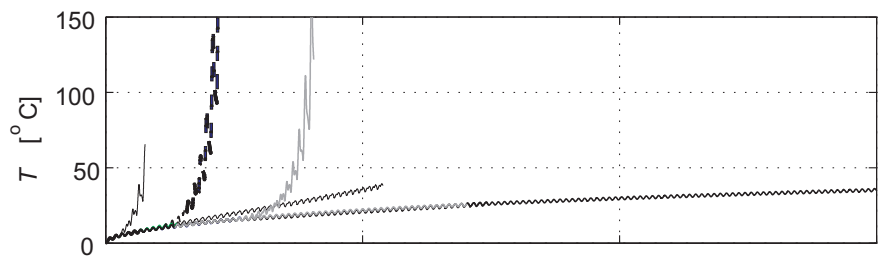

b)

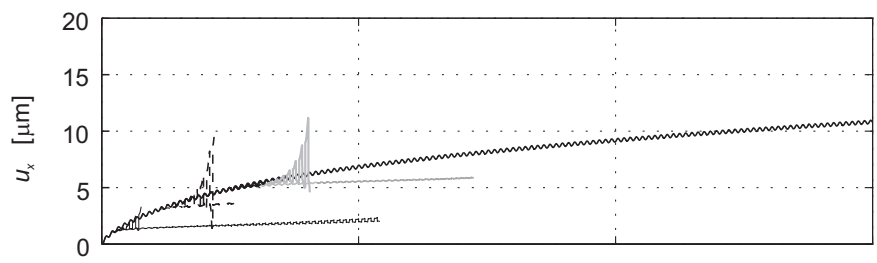

c)

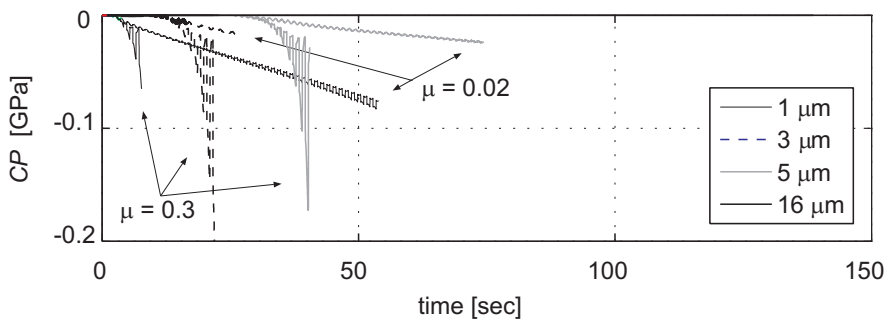

Figure 5.3: Simulation results with initial clearance $\mathrm{c}=1,3,5,16 \mu \mathrm{m}$ and two coefficients of friction, $\mu=0.02$ and 0.3 ; a) the piston temperature evolution traced at the location near the corner, see figure $5.2 \mathrm{a}, \mathrm{b}$ ) radial expansion of the piston, c) the resulting contact pressure

sufficient, meaning the lower part does not contribute to the stiffness, the contact pressure dependence can be approximated as shown in figure $5.4 \mathrm{~b}$. The performance of the simplified geometry of the U-piston is analyzed, see figure 5.5 and 5.6, considering different materials and operating conditions. In this analysis the coefficient of friction, 0.02 is assumed.

\subsubsection{The effect of operating frequency}

Simulation results with the U-piston and three different operating speed are shown in figure 5.5. Steady state can be obtained after $40 \mathrm{sec}$, with contact pressure of $10 \mathrm{MPa}$. For higher oscillating frequency, $250 \mathrm{RPM}$, more time is required to reach steady state operation. As expected, the higher sliding piston velocity generates higher temperature and consequently the contact pressure. Apparently for the 500 RPM the friction generated heat leads to thermo-mechanical instability. 
a)

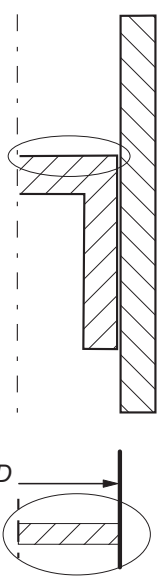

b)

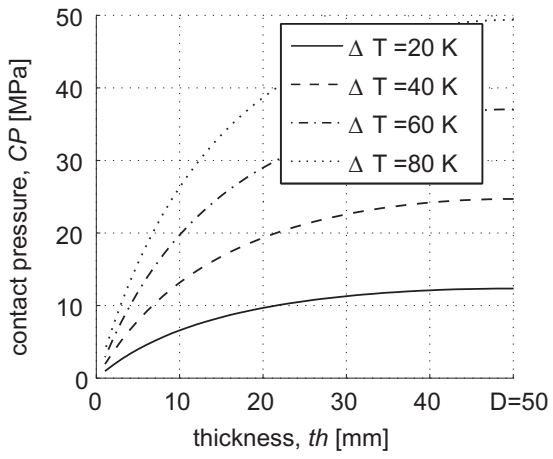

c)
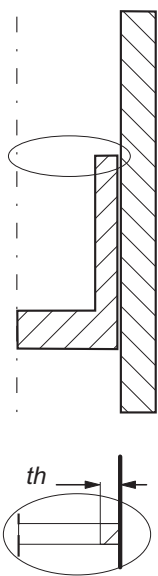

Figure 5.4: Cross view on the piston/cylinder assembly a) classical piston design, b) contact pressure on the piston/cylinder interface with respect to the wall thickness th, c) alternative piston design
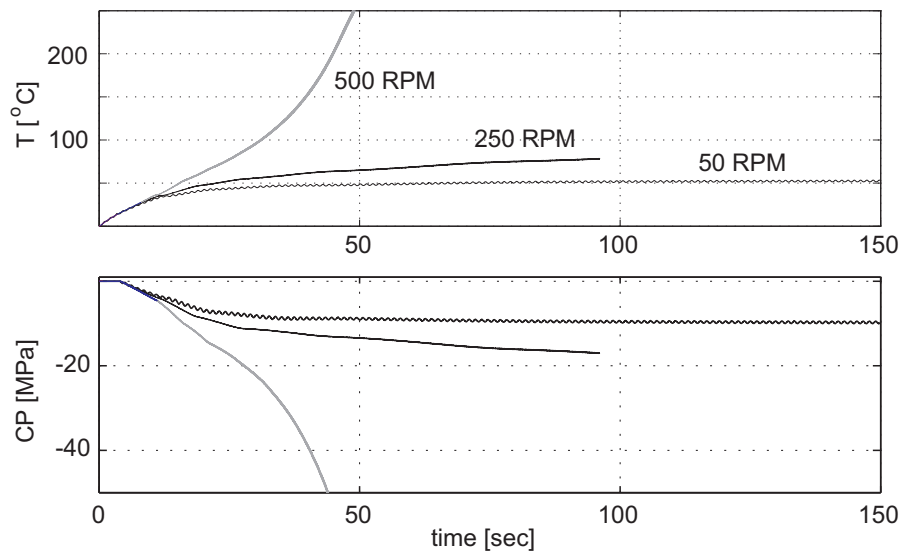

Figure 5.5: Simulation results with the U-piston, effect of the rotating speed

\subsubsection{The effect of piston material}

Figure 5.6 shows the difference in temperature and contact pressure development considering three typically used materials for pistons, the thermal and mechanical properties are given in table 5.3. Because of favorable material properties of the aluminium alloy both material temperature and contact pressure are lower compared to the other materials. The high thermal conduction efficiently conducts heat away from the sliding surface; this also results in more uniform and lower temperature 


\begin{tabular}{lcccl} 
& 52100 & AL $7075-\mathrm{T} 651$ & $316 \mathrm{~L}$ & \\
\hline elastic modulus, $E$ & 213 & 74 & 193 & {$[\mathrm{GPa}]$} \\
Poisson ratio, $\nu$ & 0.29 & 0.30 & 0.25 & {$[-]$} \\
thermal expansion coefficient, $\alpha$ & 12.0 & 22 & 16.2 & $\mathrm{x} 10^{-6}[1 / \mathrm{K}]$ \\
thermal conductivity, $\lambda$ & 46.6 & 120 & 17.0 & {$\left[\mathrm{~W} / \mathrm{m}^{2} \mathrm{~K}\right]$} \\
density, $\rho$ & 7875 & 2800 & 7990 & {$\left[\mathrm{~kg} / \mathrm{m}^{3}\right]$} \\
specific heat, $c_{p}$ & 490 & 960 & 500 & {$[\mathrm{~J} / \mathrm{kg} \mathrm{K}]$} \\
\hline
\end{tabular}

Table 5.3: Piston material properties used in the analysis
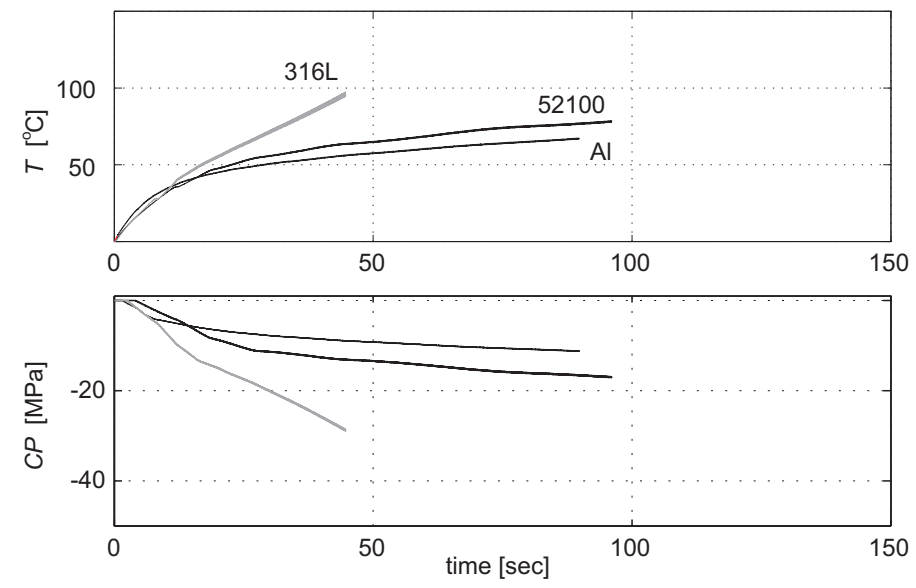

Figure 5.6: Simulation results with the U-piston, effect of different piston materials

distribution thus less material expansion. In addition, the lower elastic modulus further reduces the contact pressure. As can be already expected looking at the material data of the austenitic stainless steel, $316 \mathrm{~L}$, the situation is inverted compared to a piston made of aluminium alloy.

\subsection{Concluding remarks}

The analysis of a reciprocating piston has been done including prediction of thermomechanical instability. The finite element method with ALE formulation proved to be a useful tool in the analysis of coupled thermo-mechanical problem including sliding contact. It provides a means for studying a complex problem in detail which provides better understanding and eventually it may serve as a tool for optimization of the configuration. This is not only motivated by the results discussed in Chapter 4, but above all by the results with respect to the main goal in this thesis. It has been shown that the piston can be designed in such a way that the piston/cylinder clearance is 
eliminated during operation. This has the potential to reduce the gas leakage nearly to zero.

Performance of the U-piston was analyzed considering different operating speeds. From the results it follows that the given operating conditions, 500 RPM and initial clearance of $\mathrm{c}=5 \mu \mathrm{m}$ would lead to themo-mechanical instability. Clearly, that initial clearance is not sufficient. The difference in temperature and contact pressure development in the piston/cylinder assembly considering different materials was also demonstrated. Aluminium alloy is the best choice for piston design mainly because of the favorable material properties. 


\section{Stability analysis of a gas lubricated piston}

\subsection{Introduction}

In oil free piston compressors where low friction losses are required, solid lubricants can be used. In Chapter 3 concerning piston tribology, coatings with low friction and high wear resistance were investigated. In that design solution we assumed that sliding contact between piston and cylinder is unavoidable. In this chapter another approach is taken. The piston will be designed as a self-lubricating (aerodynamic) piston and the working gas will be used as the lubricant. Low viscosity of the gas offers virtually frictionless operation. In order to fully benefit from the low friction potential of gas lubrication any contact between the piston skirt and cylinder liner must be avoided. However, because of unbalanced forces acting on the piston, it will also move laterally in the cylinder and occasionally hit the cylinder wall. These occasional contacts cause friction, wear and also noise eventually leading to failure. A good design must therefore provide a smooth and stable reciprocating motion of the piston and ensure that the gas film separating the surfaces is maintained during operation. At the same time the gas leakage along the piston needs to be limited. The sealing of the compressed gas in this case is obtained by the small radial clearance between piston and cylinder only, also called a clearance seal. To optimize the piston design a complete dynamic analysis of the reciprocating piston is needed. The analysis requires simultaneous solution of the equations of motion of the piston along with the Reynolds equation for the lubricating gas film. For this purpose a numerical model has been developed for analysis of the secondary piston motion (SPM) of a gas lubricated ringless piston. It takes into account the piston geometry, dynamic properties of the piston and operating conditions. The influence of various design parameters and operating conditions on the SPM has been analyzed in the application to the gas spring test set-up presented in Chapter 2. 


\subsection{Gas lubrication}

In gas lubrication, two surfaces are separated with a thin layer (film) of gas. Besides protecting the surfaces from wear, the layer also provides a smooth movement of the surfaces which eliminates problems with stick-slip conditions present in boundary lubrication.

Some strengths and weaknesses of gas lubricated bearing compared to oil bearing are as follows:

- friction or viscous resistance is extremely low

- the lubricant is ample and clean

- gas operates well from high to low temperatures

- no thermal heating with high sliding speed

- for the same bearing size the load carrying capacity of gas bearing is many times less

- surface relative speed must be high

- alignment and dimensions must be extremely accurate

- surface finish must be of high quality

- less damping, stability characteristics are poor

In mechanical engineering, gas lubricated bearings are applied whenever it is necessary to have an extremely low friction or a clean environment. Examples can be found in:

- metrology, measurement units where precision and constancy of torque are critical

- high-speed precision machinery where precision of positioning is required and stick-slip or vibration need to be avoided. High speed grinding spindle, $(\mathrm{f}=$ $2000 \div 5000 \mathrm{~Hz}$ ) used for high speed production

- computer devices, such as tapes, discs containing read-write heads 'flying' with an air gap

- in food and textile processing machinery, where cleanliness and absence of contaminates are critical,

- medical applications, for example high-speed dental drills

- foil type gas bearings are used for turbo charged power generating units in aircraft and spaceborne applications.

The applications of gas bearings mentioned above can be divided into three categories; externally pressurized (aerostatic) bearings, self-acting (aerodynamic) bearings and hybrid bearings which are combinations of aerostatic and aerodynamic bearings. 


\section{Aerostatic lubrication}

a)

b)

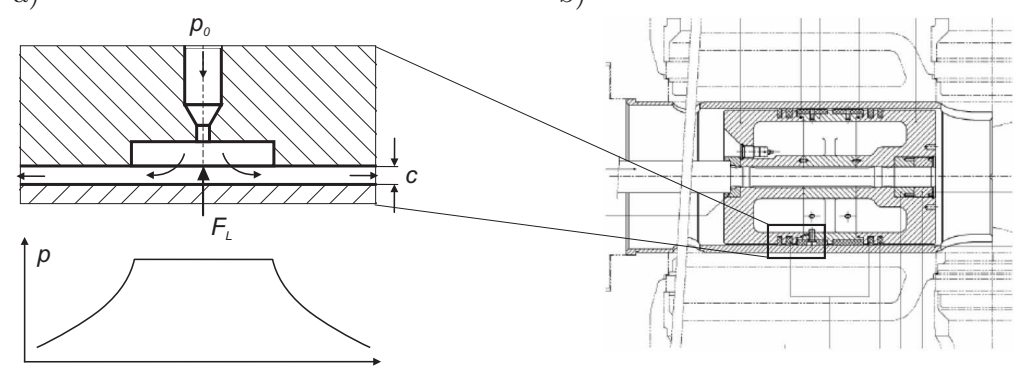

Figure 6.1: Aerostatic lubrication

Externally pressurized gas bearings are used most frequently. A compressed gas is used to generate a lift force that prevents contact between the sliding surfaces. Here relative motion of the surfaces is not required. Figure 6.1a shows schematically aerostatic bearing and pressure distribution. Compressed gas with pressure $p_{0}$ is supplied which generates the aerostatic lift force $F_{L}$. The technology of aerostatic lubrication is applied by Thomassen in the so-called 'free floating piston compressor' [55]. The compressor is shown in figure $6.1 \mathrm{~b}$.

$$
F_{L}=\int_{S} p d S
$$

\section{Aerodynamic lubrication}

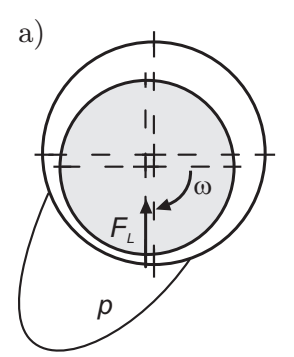

b)

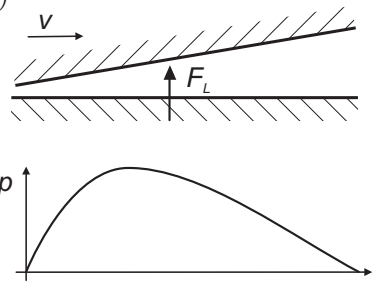

c)

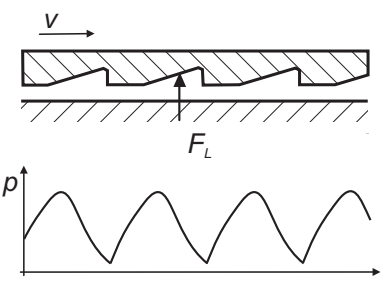

Figure 6.2: Examples of aerodynamic lubrication; a)journal and b,c) plane bearing with pressure distributions

In aerodynamic bearings the force separating two sliding surfaces is generated due to relative motion of the surfaces. If the shape of the surfaces forms a converging gap, a so-called bearing wedge, the gas flowing into the gap is being compressed which 
generates the aerodynamic lift force $F_{L}$. Figure 6.2 schematically shows principles of the aerodynamic lubrication in different applications.

The aerodynamic forces depend solely on the pressure distribution. Gas lubrication has specific characteristics due to compression of the lubricant. Firstly, gas has a very low viscosity, approximately $2 \cdot 10^{-5} \mathrm{~Pa} \cdot \mathrm{s}$ for air under room conditions, compared to oil with approximately $2 \cdot 10^{-3} \mathrm{~Pa} \cdot \mathrm{s}$. Therefore with aerodynamic lubrication lower bearing loads can be achieved for the same bearing dimensions. At the same time the viscous resistance is considerably lower. Secondly, the compressibility of the gas limits the pressure build-up. This is also related to rather low stiffness and damping ability of gas bearings. On the other hand, the separation between asperities of the sliding surfaces is also correspondingly smaller so that special precaution must be taken in manufacturing the bearing. Most gasses lack boundary lubricating properties, therefore damage can easily occur when the sliding surfaces touch. The critical situations are during starting/stopping or the extreme piston positions (TDC and $\mathrm{BDC})$ when the velocity is too low to lift the surfaces.

The bearing surfaces may have different geometries. The distance between the bearing surfaces is called the film thickness $h$. In the present work various piston geometries will be analyzed. It is assumed that the surfaces are undeformable, so mechanical and thermal deflections are negligible. This is a rather severe assumption since in reality thermal distortion may be considerable, see Chapter 4. Including those effects would complicate the problem to a large extent. It is believed, however, that the imposed simplification will not affect the validity of the parametric study too much.

\subsection{Literature survey}

Lubrication characteristics between piston and cylinder in reciprocating motion has been a well-explored topic. Mainly, investigations were carried out in the application to oil lubricated piston engines. However, some studies were also done on gas lubricated compressors. A brief survey, focusing on those contributions relevant to the present investigation, will now be presented.

\section{Oil lubricated piston}

Already before the eighties of the previous century, Li et al. [61], studied theoretically and experimentally piston lubrication. Those authors have shown through an analytical model that piston skirt friction can be significantly reduced if the piston center of rotation is located in a favorable position. Zhu et al. [88] numerically investigated piston motion, lubrication, and friction in mixed lubrication regime taking into account effects of surface waviness, roughness, surface profile, bulk elastic deformation, and thermal distortion of both piston skirt and cylinder bore. The proposed model was applied to a four-stroke automotive engine showing that good hydrodynamic lubrication can minimize the possibility of piston impact against the cylinder bore and reduce the frictional loss. Theoretical and experimental results were 
obtained by Yamaguchi [87] for two piston-cylinder assemblies. One operating in the standard mode, with hydrodynamic lubrication, and another operating in hydrostatic lubrication with oil being injected in the radial clearance between piston and cylinder. It was shown for low crankshaft velocities, that the hydrostatic piston can operate with reduced friction and same oil leakage compared to the hydrodynamic piston as long as the radial clearance is made small.

\section{Gas lubricated piston}

Mourelatos [65] determined the feasibility of using gas bearing technology to separate the piston from the cylinder liner in a low heat rejection (LHR) engine. An efficient method was presented to solve the transient two-dimensional, compressible Reynolds equation for a gas bearing based on FEM. The studies showed that under certain conditions a ringless piston with a carefully designed piston skirt profile can support a side load of several Newtons running on a gas film. It was shown also that the piston load capacity increases drastically if the piston is tapered instead of cylindrical. The LHR engine operates under elevated temperatures, (approx $500{ }^{\circ} \mathrm{C}$ ) and none of the commonly used lubricant oils could withstand these temperatures.

In a sequence of three papers [29] [37] [38] Gommed and Etsion presented a mathematical model for analyzing the dynamics of gas lubricated pistons. A relatively large size cryocooler was investigated (piston diameter $80 \mathrm{~mm}$, stroke $20 \mathrm{~mm}$ ) and it was shown that although a cylindrical piston shape is commonly used for ringless piston applications, this is not the best choice for an optimum design as far as piston stability and sealing performance are concerned. Other piston shapes were explored and improved design was obtained with non-cylindrical profiles.

\subsection{Problem formulation}

The piston, with radius $R$ and length $L$ is driven in reciprocating motion by the action of the connecting rod driven by the rotating crankshaft. The cycle starts at the bottom dead center (BDC), where $\phi_{c}=0$ and ends at the same point after one revolution. The angular speed $\omega$ is constant. The primary piston motion i.e. along the cylinder axis; position, velocity and acceleration are functions of the crank angle $\phi_{c}$ and can be readily determined. The secondary piston motion i.e. the piston lateral translations and rotations within the clearance is very limited but will be essential in this analysis. The piston axial velocities and acceleration characteristics are shown in figure $6.3 \mathrm{~d}$. The gas pressure boundary conditions are simplified to sinusoidal variations. The pressure above the piston $p_{a}$ changes with an amplitude as for gas compression ratio 2 . The pressure below the piston $p_{b}$ varies as well because that space changes its volume due to the pistons diameter difference. The pressure characteristics are shown in figure $6.3 \mathrm{~d}$. The buffer absolute pressure $p_{0}$ is taken equal to $0.1 \mathrm{MPa}$. Piston orientation within the cylinder bore is shown schematically in $6.3 \mathrm{~b}$. 
Geometric parameters

\begin{tabular}{lcl}
\hline cylinder diameter, $2 R$ & 0.05 & {$[\mathrm{~m}]$} \\
piston length, $L$ & 0.05 & {$[\mathrm{~m}]$} \\
piston center of mass, $c \mathrm{mr}$ & 0.04 & {$[\mathrm{~m}]$} \\
piston stroke, $S$ & 0.052 & {$[\mathrm{~m}]$} \\
\hline Dynamic properties & & \\
\hline piston mass, $m$ & 0.6 & {$[\mathrm{~kg}]$} \\
piston moment of inertia, $I$ & $3.210^{-4}$ & {$\left[\mathrm{~kg} \mathrm{~m}{ }^{2}\right]$} \\
\hline Operating conditions & & \\
\hline piston frequency, $f$ & 25 & {$[\mathrm{~Hz}]$} \\
gas viscosity, $\eta$ & $2.210^{-5}$ & {$[\mathrm{~Pa} \cdot \mathrm{s}]$} \\
gas density, $\rho$ & 2.75 & {$\left[\mathrm{~kg} / \mathrm{m}^{3}\right]$} \\
\hline
\end{tabular}

Table 6.1: Characteristic data

\subsection{Secondary piston motion model}

The Secondary Piston Motion (SPM) model consists of solving a system of equations describing the gas flow in a thin gap, which is the Reynolds equation accompanied by the equation for the gas film thickness and the equations of piston motion. The equations are discussed below.

\subsubsection{Gas flow model}

The gas pressure in the film generates lateral forces on the piston which are essential for the SPM analysis. An accurate method for prediction of their variations is needed. The analysis method consists of well-known theories for a gas flow in a narrow gap and a numerical solver to obtain a solution for the resulting equations. The general equations that describe the flow of viscous compressible fluid are the equations of mechanics and thermodynamics. Extensive description can be found in [10].

The following assumptions are made in the model. The radial variations of gas density, viscosity and pressure across the small clearance are neglected. The gas flow along the clearance is laminar and subsonic. Entrance effects into the clearance are neglected. Gas shear forces acting on the piston wall are negligible compared to all other forces acting on the piston.

\section{Basic equations}

It is assumed that the working gas in the cylinder behaves as an ideal gas under isothermal compression. The equation of state is given as:

$$
p=\rho R_{g} T
$$



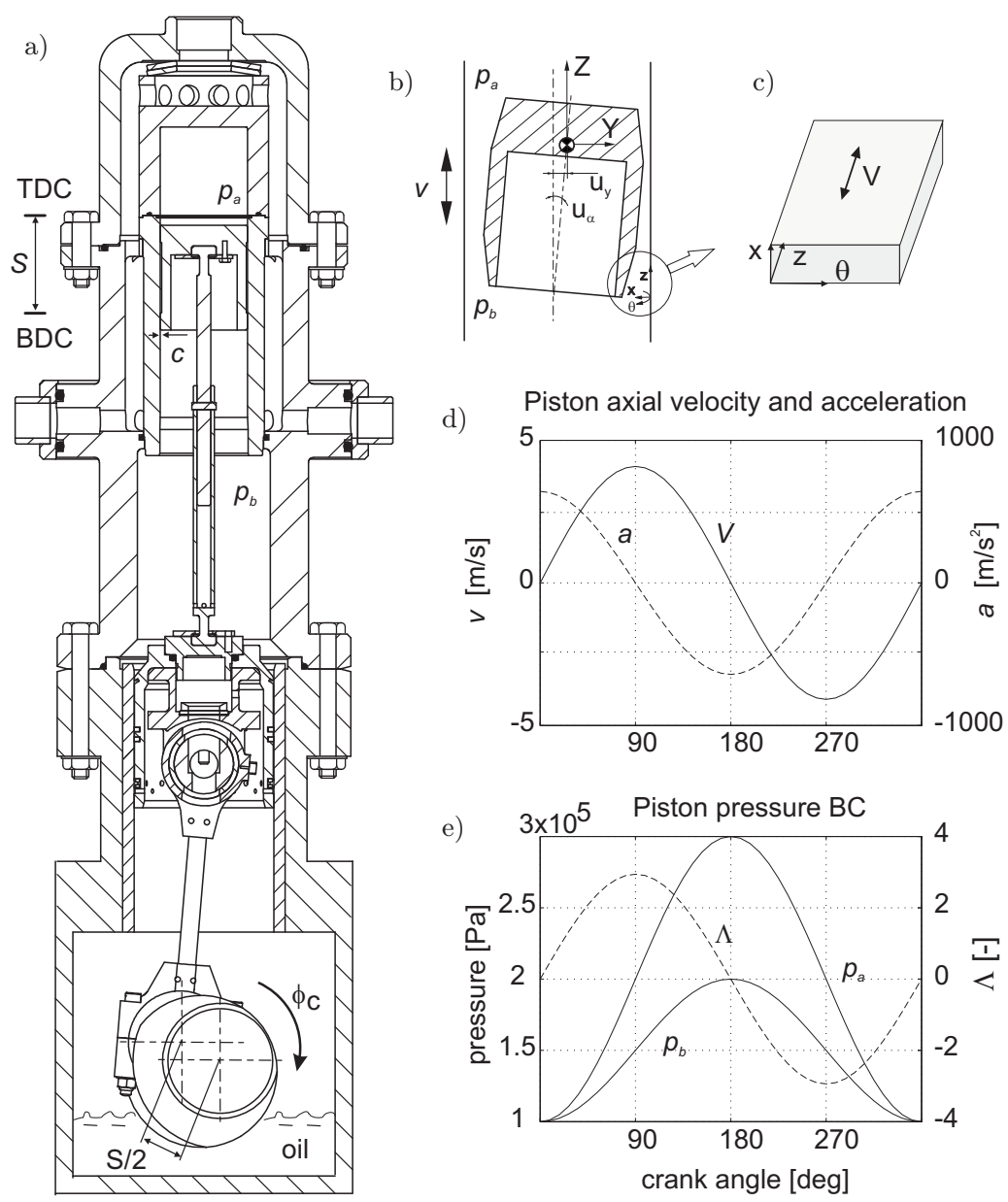

Figure 6.3: a) The test set-up b) piston orientation c) lubricated surfaces and the coordinate system d) piston axial velocity and acceleration e) compression space and buffer gas pressure change during cycle

where $p$ is the gas pressure, $\rho$ stands for the gas density, $R_{g}$ is the universal gas constant and $T$ is the temperature.

In non-steady state conditions the fluid density may change with time. This is taken into account considering mass flow. If the density $\rho$ is the same through the film thickness the continuity of mass flow $q$ is given by the equation:

$$
\frac{1}{R} \frac{\partial}{\partial \theta}\left(\rho q_{\theta}\right)+\frac{\partial}{\partial z}\left(\rho q_{z}\right)+\frac{\partial}{\partial t}(\rho h)=0
$$

Considering a force balance of an element of fluid which exhibits a proportional strain rate to the shear stress $\tau=\eta \partial u / \partial x$ (Newtonian fluid), where $\eta$ denotes the gas 
viscosity and $x$ is the coordinate in the film thickness direction, the force balance gives the following equation:

$$
\frac{\partial p}{\partial z}=\frac{\partial}{\partial x}\left(\eta \frac{\partial u}{\partial x}\right)
$$

Integration of the equation (6.4) with the assumption that the fluid speed at the surface is the speed of the surface itself leads to the equation of gas velocity $v_{z}$ in the film:

$$
v_{z}=\frac{\partial p}{2 \eta \partial z}\left(x^{2}-x h\right)+v \frac{x}{h}
$$

The sum velocity $v$ equals the sum of the velocities of the two surfaces, i.e. $v=v_{1}+v_{2}$, where $v_{2}=0$. In the present study the sum velocity is equal to piston velocity which is changing sinusoidally. The flow rate in $z$ direction per unit width of $R \theta$ is achieved by the integration $q_{z}=\int_{0}^{h} v_{z} d x$ which gives

$$
q_{z}=\frac{-h^{3} \partial p}{12 \eta \partial z}+v \frac{h}{2}
$$

\section{Reynolds equation}

In aerodynamic lubrication, the thickness of the lubricant film between the surfaces is generally small compared to the characteristic dimensions of the contact area. A force balance of an element of fluid and mass continuity of fluid, the model describing the flow of a Newtonian fluid, reduces to a single equation that relates the pressure in the gap to its geometry. If the velocities of both surfaces are in the z-direction only, the Reynolds equation reads:

$$
\frac{1}{R^{2}} \frac{\partial}{\partial \theta}\left(h^{3} p \frac{\partial p}{\partial \theta}\right)+\frac{\partial}{\partial z}\left(h^{3} p \frac{\partial p}{\partial z}\right)=6 \eta v \frac{\partial}{\partial z}(h p)+12 \eta \frac{\partial}{\partial t}(h p)
$$

where $p=p(\theta, z, t)$ is the hydrostatic pressure above ambient pressure and $h=$ $h(\theta, z, t)$ is the gap between the sliding surfaces. The terms on the right-hand side are generally referred to as the wedge term and the squeeze term according to the mechanism of pressure generation they represent. Flow induced by the wedge term is also known as Couette flow. Pressure induced flow, as on the left-hand side of the equation, is usually termed Poiseuille flow. The Reynolds equations is often analyzed in its dimensionless form where a bearing number $\Lambda$ represents the dimensionless velocity,

$$
\Lambda=\frac{6 \eta v L}{h_{2}^{2} p_{0}}
$$

where $L$ is the bearing length, $h_{2}$ and $p_{0}$ are the minimum gas film thickness and pressure. The bearing number is often used as a characteristic value of the analyzed gas bearing. When the bearing number increases the bearing load capacity increases as well. Gross et al. [40] have shown that the compressibility of the gas can be neglected in cases where the bearing number becomes very low. This effect is also 

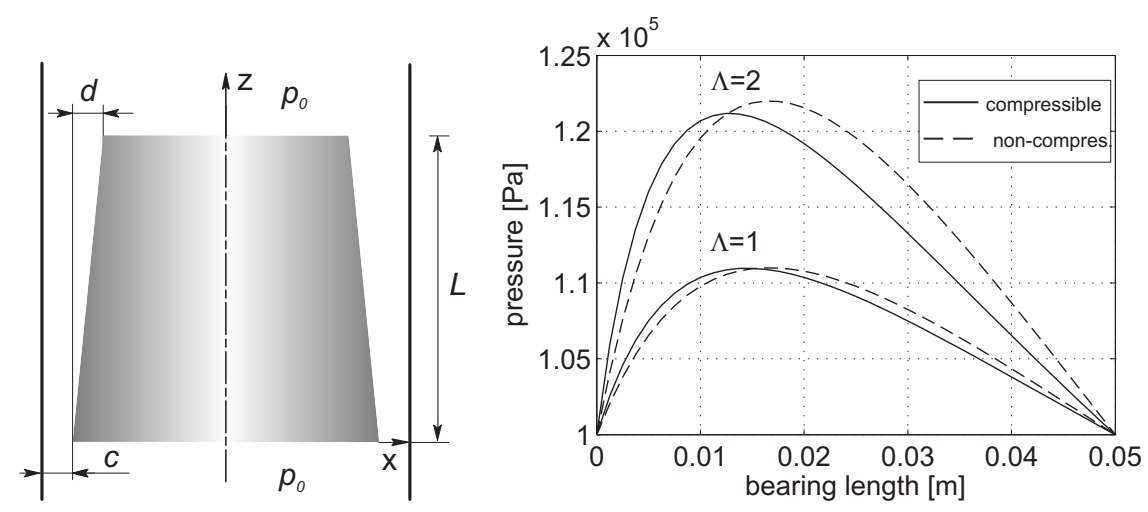

Figure 6.4: Wedge bearing and the pressure distribution

illustrated in figure 6.4 which shows the pressure distribution dependence in respect to a different bearing number.

The equation (6.7) can be solved only numerically. Different numerical methods were developed based on finite difference and finite element method. The advantage of FEM is that it can be applied to various geometries. Nevertheless, the FDM is also widely used especially for regular domains. The Reynolds equation to be solved requires conditions on the boundary enclosing the spatial domain. Figure $6.3 \mathrm{~d}$ and 6.3e shows the BC's where the piston velocity and pressures are given as a function of a crank angle. In this work the governing equation is solved with FDM with the grid and coordinate system as shown in figure 6.5. The nonlinear problem in $p$ is first linearized according to the Newton's method, [84]. As a consequence the nonlinear problem in $p$ is reduced to solving a linear partial differential equation in $\delta p$, equation (6.9) in order to determine the value that improves the accuracy of the estimate $p$.

$$
\begin{aligned}
& \frac{1}{R^{2}} \frac{\partial}{\partial \theta}\left(h^{3} p \frac{\partial \delta p}{\partial \theta}+\delta p h^{3} \frac{\partial p}{\partial \theta}\right)+\frac{\partial}{\partial z}\left(h^{3} p \frac{\partial \delta p}{\partial z}+\delta p h^{3} \frac{\partial p}{\partial z}\right)-6 \mu v \frac{\partial \delta p h}{\partial z}-12 \eta \frac{\partial \delta p h}{\partial t}= \\
& -\frac{1}{R^{2}} \frac{\partial}{\partial \theta}\left(h^{3} p \frac{\partial p}{\partial \theta}\right)-\frac{\partial}{\partial z}\left(h^{3} p \frac{\partial p}{\partial z}\right)+6 \mu v \frac{\partial p h}{\partial z}-12 \eta \frac{\partial p h}{\partial t}
\end{aligned}
$$

The Newton-Raphson (NR) algorithm [1] is used to solve the resulting system of equations.

\subsubsection{Equations of motion}

The piston motion in $z$-direction is defined. The compressed gas forces acting on the piston $p_{a}$ and $p_{b}$ and piston inertia forced are balanced by the driving force $F_{\mathrm{cr}}$. The piston radial motion is caused by the action of pressure in the gas film. The piston free body diagram and the coordinate system used to compute the secondary motion 
a)

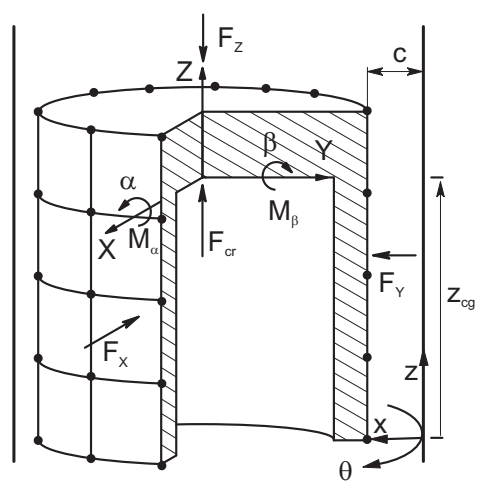

b)

(L)

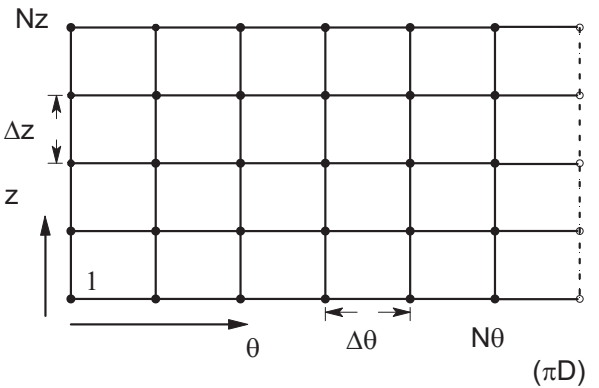

Figure 6.5: a) Free body diagram with the used coordinate systems, b) the grid used for FDM solution of the Reynolds equation

is depicted in figure 6.5. The origin of the coordinate system is located in the center of gravity of the piston. The piston is free to translate in $x$ and $y$-direction and to rotate around $x$ and $y$ axis. The $u$ vector contains the displacement and rotation components $u=\left(u_{x}, u_{y}, u_{\alpha}, u_{\beta}\right)$. The equilibrium equation can be written as:

$$
M \ddot{u}=F
$$

where $M$ is the mass matrix containing the piston mass and piston moment of inertia about the center of gravity. The transient forces $F=\left(F_{x}, F_{y}, M_{\alpha}, M_{\beta}\right)$ exerted by the lubricating gas pressure $p$ can be obtained from integration of the pressure distribution around the piston.

$$
\begin{array}{r}
F_{X}=-\int^{2 \pi} \int^{L} p(\theta z) R \cos \theta d z d \theta \\
F_{Y}=-\iint p(\theta z) R \sin \theta d z d \theta \\
M_{\alpha}=\iint z p(\theta z) R \sin \theta d z d \theta \\
M_{\beta}=-\iint z p(\theta z) R \cos \theta d z d \theta
\end{array}
$$

For uniform grid the forces can be written in matrix form:

$$
F=H^{T} p d A
$$

\subsection{Numerical procedure}

The theoretical model described in the previous section is used to calculate the trajectory of the ringless piston within the cylinder clearance under given operating 
conditions. The problem consists of finding the piston radial displacements $u_{x}, u_{y}$ and rotations $u_{\alpha}, u_{\beta}$ as a function of time. The algorithm is summarized in the flow chart, see figure 6.6.

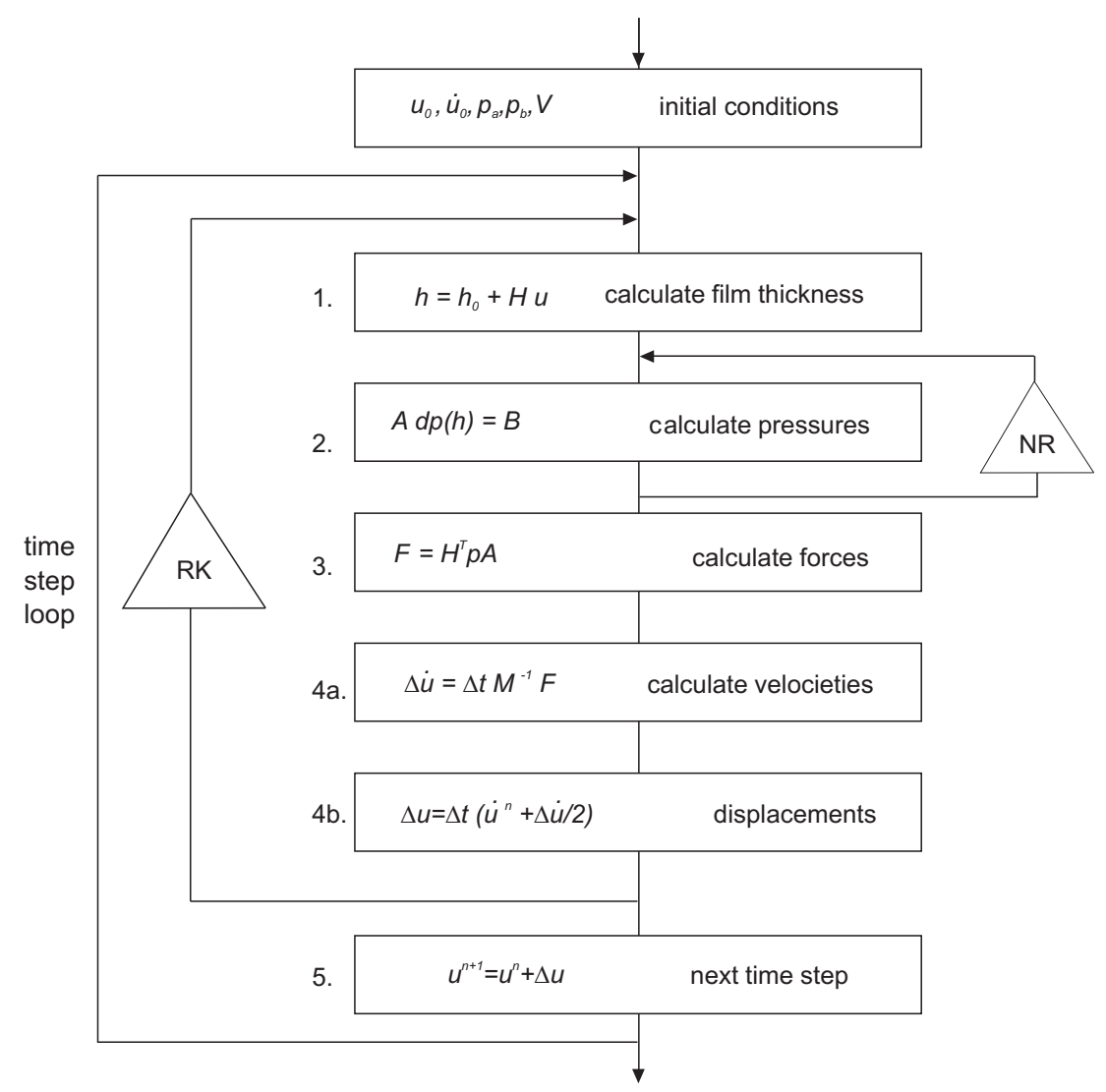

Figure 6.6: Algorithm of the SPM model

1. Assuming an initial piston position within the cylinder the vector containing gas film thickness $h$ can easily be derived with the following equation,

$$
h(z, \theta)=c(z)-u_{x} \cos (\theta)-u_{y} \sin (\theta)+u_{\alpha} \sin (\theta) z-u \beta \cos (\theta) z
$$

where $c$ is the initial piston clearance. For a straight piston surface this is a constant value, and for the complex geometry the clearance will depend on the piston shape. In the Reynolds equation (6.7) the derivatives with respect to $z$ and time are also needed and can be derived as follows:

$$
\frac{d h}{d z}=\frac{d c}{d z}+u_{\alpha} \sin (\theta)-u_{\beta} \cos (\theta)
$$




$$
\frac{d h}{d t}=-\dot{u}_{x} \cos (\theta)-\dot{u}_{y} \sin (\theta)+\dot{u}_{\alpha} \sin (\theta) z-\dot{u}_{\beta} \cos (\theta) z
$$

2. For the given film thickness and the BC's at the time $t=t^{n}$ one can calculate the pressure distribution on the piston skirt $p$. The aerodynamic pressure developed in the gas film is obtained from the transient compressible Reynolds equation (6.7) for one direction relative surface velocity $v$.

3. The transient forces $F_{X}, F_{Y}$ and moments $M_{\alpha}, M_{\beta}$ exerted by the gas pressure can be obtained from integration of the pressure distribution around the piston, equations (6.11), on the flow chart this equation is given in matrix form.

4. The equations of motion (6.10) can now be solved to find accelerations. From the accelerations the velocities $4 \mathrm{a}$. and displacements $4 \mathrm{~b}$. can be calculated. This gives the film thickness and its derivatives $\partial h / \partial t$ and $\partial h / \partial z$ for the next time step $t+\Delta t$.

The implicit fourth order Runge-Kutta (RK) method is employed to solve the nonlinear system of equations of motion. Based on the characteristics of the operating conditions the piston $\mathrm{BC}$ are estimated for $t=t+\Delta t$. An iterative process is then needed to determine the piston radial position and velocities at $t+\Delta t$, that is the $u^{t+\Delta t}$ and $\dot{u}^{t+\Delta t}$. Based on the forces obtained from (6.11) the increment of the velocity vector $\Delta v$ is calculated that contains the linear and angular components.

$$
\begin{array}{r}
\Delta \dot{u}=\Delta t \cdot M^{-1} \cdot F ; \\
\Delta u=\Delta t\left(\dot{u}^{n}+\Delta \dot{u} / 2\right) ;
\end{array}
$$

where $F$ is the force vector including the components defined by equations (6.11).

According to the Runge-Kutta procedure the value at $t^{n+1}$ is determined by the present value, at $t^{n}$ plus the product of the size of the interval $\Delta t$ and an estimated slope (6.16) is defined as:

$$
u^{n+1}=u^{n}+\Delta t / 6\left(\Delta u_{1}+2 \Delta u_{2}+2 \Delta u_{3}+\Delta u_{4}\right)
$$

5. In the new step for the given piston position and velocities at a time step $t^{n+1}$, the film thickness is calculated and the Reynolds equation can be solved for $p$ at $t+\Delta t$. As an initial guess the $p$ at previous time $t$ is used. From many test runs it was found that the maximum time step $\Delta t=2 \mathrm{e}-5$ is required. One full cycle $(\mathrm{T}=0.04 \mathrm{sec})$ then needs 2000 steps. Convergence of the Newton-Raphson algorithm at each time step was achieved at most in two iterations. Analysis of the results indicated that if a larger time step is chosen convergence problems arise when large pressure gradients are computed on the piston skirt near the edges of the profile. The high pressure gradients are caused at the locations of low gas film thickness corresponding to high bearing number. 


\subsection{Results}

Various piston skirt geometries were investigated with the aim to find a design that will guarantee contactless operation of the piston with a minimum gas leakage. The analyzed piston skirt geometries are shown in figure 6.7. The question we are trying to answer is whether it is possible to design a piston so that when a certain initial piston misalignment is introduced the piston can be self-corrected by the hydrodynamic forces during operation. This leads to a stable operation. The piston trajectory is used to estimate the piston's ability of reducing and stabilizing its secondary motion. Considering the experimental set-up discussed in Chapter 2, this is a practical question since a certain misalignment in the piston/cylinder assembly will always be present during assembly. We define a stable piston design that allows centering its position during operation.

Further, the gas flow in the clearance was analyzed and gas leakage out of the compression space was estimated. The escaped gas volume integrated over a cycle is a quantitative measure of the piston performance. In order to provide some validation of the model and to illustrate its usefulness a series of parametric studies were performed on different piston design aspects [69].

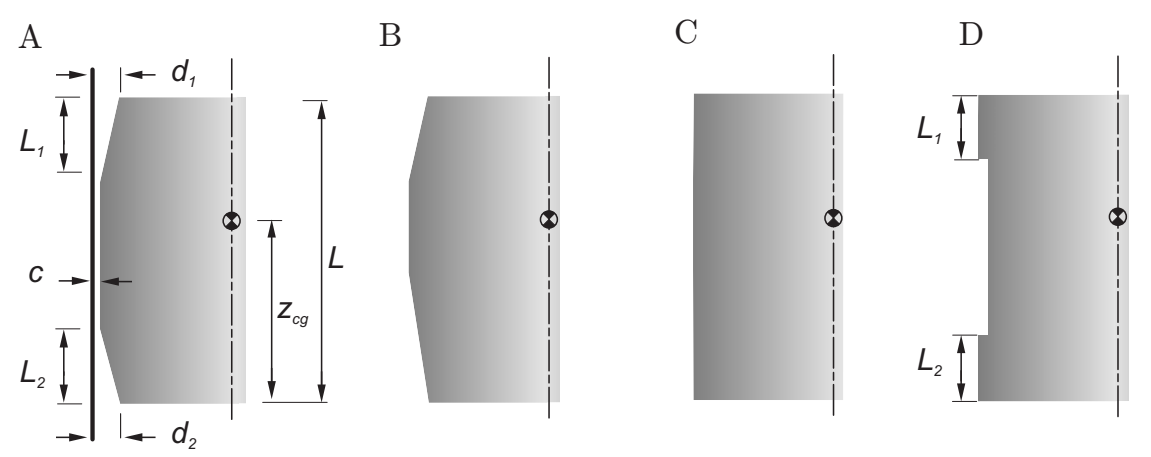

Figure 6.7: Analyzed piston geometries, $L=50 \mathrm{mmm:} \mathrm{A)} \mathrm{piston-tapered,} \mathrm{symmetric}$ $\mathrm{B})$ piston-tapered, nonsymmetric (a special case of piston A), C) cylindrical, D) test model

\begin{tabular}{llllll} 
& $\begin{array}{l}L_{1} \\
{[\mathrm{~mm}]}\end{array}$ & $\begin{array}{l}L_{2} \\
{[\mathrm{~mm}]}\end{array}$ & $\begin{array}{l}d_{1} \\
{[\mu \mathrm{m}]}\end{array}$ & $\begin{array}{l}d_{2} \\
{[\mu \mathrm{m}]}\end{array}$ & $\begin{array}{l}c \\
{[\mu \mathrm{m}]}\end{array}$ \\
\hline Piston A & 10 & 10 & 100 & 100 & 10 \\
Piston B & 10 & 20 & 40 & 1000 & 10 \\
Piston C & - & - & - & - & 10 \\
Piston D & 10 & 10 & 100 & - & 10 \\
\hline
\end{tabular}

Table 6.2: Chosen characteristic piston dimensions 


\subsubsection{Gas leakage}

In addition to the contactless operation, the gas leakage across the piston is of great interest. The variation of piston location in the cylinder as well as the sinusoidal nature of the operating conditions result in time-dependent gas flow rate across the piston. The instantaneous volumetric gas leakage through the clearance between the piston and cylinder is obtained by integrating the gas flow, equation (6.6) on the piston circumference:

$$
q_{z}=\int_{0}^{2 \pi}\left(\frac{-h^{3} \partial p}{12 \eta \partial z}+v_{p} \frac{h}{2}\right) R d \theta
$$

From that, cycle average leakage can be calculated,

$$
Q_{z}=-\frac{1}{T} \int_{0}^{T} q_{z} d t
$$

The gas leakage is strongly affected by the gas film thickness and the pressure gradient. For a clearance seal this means the more the piston displaces radially the higher the gas leakage. A gas flow is considered in the clearance seal shown in the figure $6.8 \mathrm{a}$ where a static pressure gradient $\Delta p / L$ is $2 \mathrm{MPa} / \mathrm{m}$ and zero relative velocity. It is clear from the results presented in the figure $6.8 \mathrm{~b}$ that higher eccentricity involves larger gas leakage. However, a piston tilt involves decreasing gas flow which is explained by the decreasing gas film thickness. In both cases, only small perturbations are negligible. Considering the radial perturbation, the piston needs to be displaced 2 $\mu \mathrm{m}$. Consequently, for more unstable piston regarding lateral motion one can expect higher gas flow. Yet, an angular piston motion can reduce the gas flow.

a)

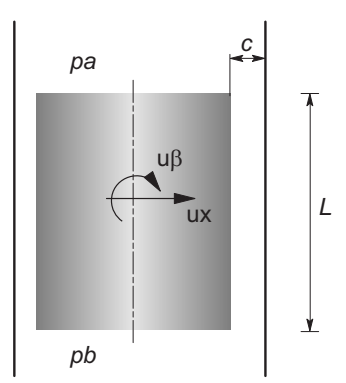

b)

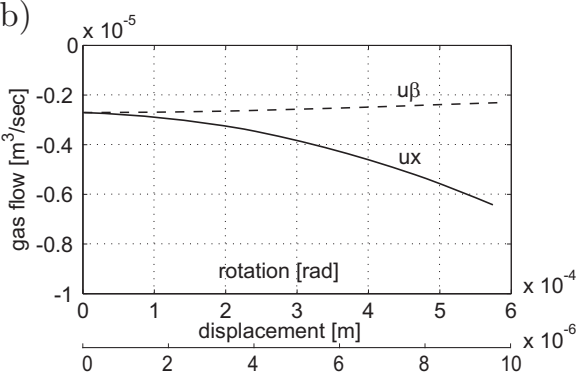

Figure 6.8: a) clearance seal and b) volumetric gas flow vs piston radial displacement and piston rotation

\subsubsection{Parametric study}

\section{Piston geometry}

Figure $6.9 \mathrm{a}$ and $\mathrm{b}$ shows solutions for the piston trajectory represented by the radial displacement and tilt with the corresponding force and moment, $6.9 \mathrm{c}$ and $\mathrm{d}$. The time 
$t=0$ corresponds to the BDC. At this position the piston is tilted in the XY plane $u_{\beta}=1.510^{-4} \mathrm{rad}$.
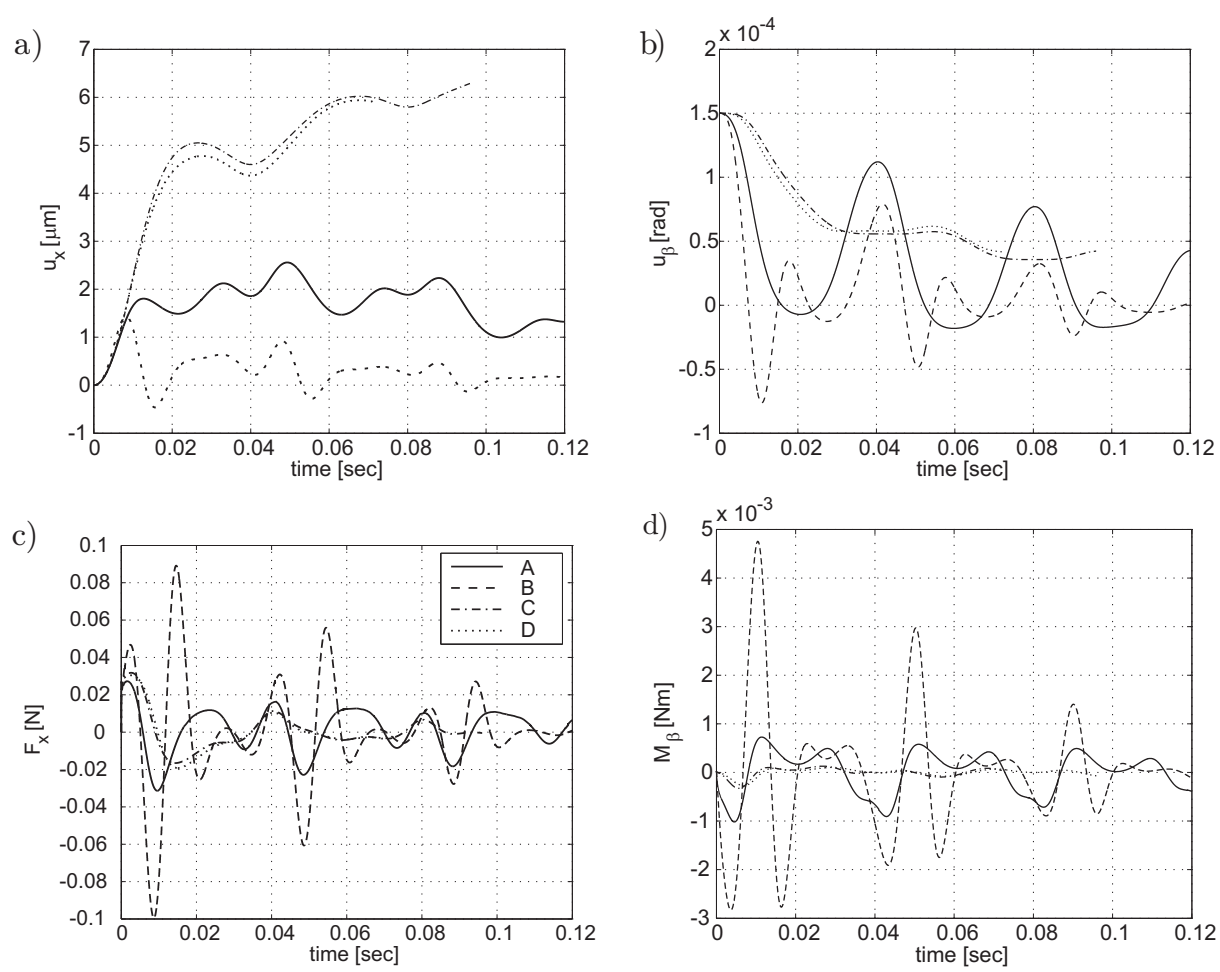

Figure 6.9: Results of SPM analyses for the four piston geometries

In general it can be observed that the characteristics differ considerably on the piston skirt profiles. In case of the piston $C$ and $D$ the restoring forces are too weak to gain a stable motion. In both cases the forces gradually move the piston away from the center. Consequently, the profiles $A, B$ and $C, D$ are classified as respectively stable and not stable designs.

The forces in the middle of the piston stroke mainly move the piston to the center. This is caused by the aerodynamic lubrication action due to the high piston velocity. This effect is observed in all the stable cases. As the piston moves away from that location the aerodynamic effects become smaller and the forces drop. However the lubrication becomes dominated by aerostatic lubrication due to the increasing pressure gradient along the piston skirt. Whenever the piston approaches the cylinder wall the film thickness drops and consequently the aerodynamic forces increase. This situation corresponds to higher bearing number as shown for a plane wedge slider bearing, see figure 6.4. The closer to the wall, the more important becomes the squeeze film effect and will contribute to the repulsive forces. At the BDC the side forces can only be present because of this action. Away from the two dead centers all the effects are 
a)

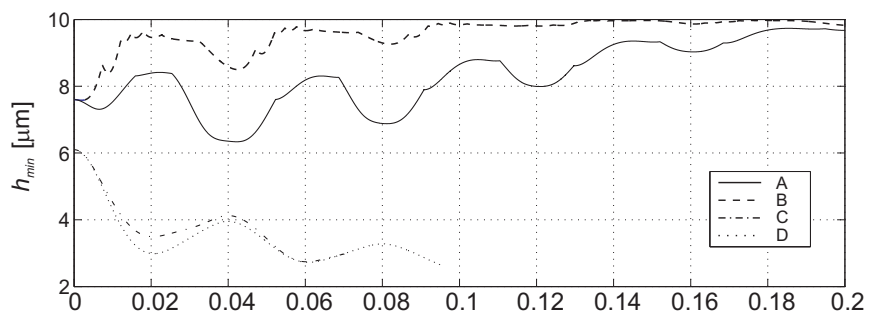

b)

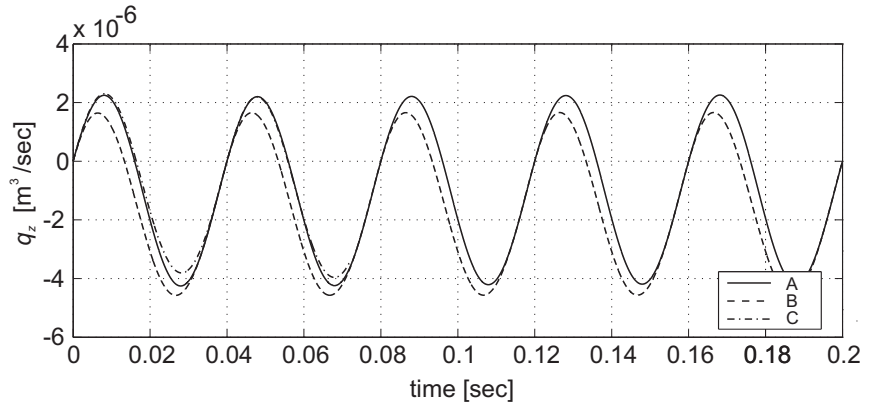

Figure 6.10: a) Minimum film thickness value variation and b) instantaneous volumetric gas leak

important hydrostatic bearing, squeeze film and wedge film.

Analyzing the forces and displacements in figure 6.9 it can be seen that initially the forces rapidly increase, resulting in positive displacements. Within the half stroke of the piston until the time $t=0.01 \mathrm{sec}$ the forces gradually weaken and change direction, in case of piston $\mathrm{A}$ and $\mathrm{B}$. For the profiles $\mathrm{C}$ and $\mathrm{B}$ the direction would be changed later in time, in the second half of the stroke. This is explained by the piston tilt in the reversed direction, see figure $6.9 \mathrm{a}$, upon the negative moment produced by the hydrodynamic pressure, see figure $6.9 \mathrm{~b}$. This means that the piston and cylinder axis are tending to be more parallel. Consequently, the gas film wedge is reduced so the hydrodynamic bearing forces are reduced as well. The negative side forces at half stroke of the piston result in constrained radial motion with a different degree depending on the force level. The profile A gains the highest side force which reduces the piston radial displacements considerably, gradually damping the motion in successive cycles. The profile B gains lower side forces and the reduction in radial displacement is less effective. As can be seen during the first three cycles the side forces are relatively weak and the piston remains eccentric, see figure $6.9 \mathrm{~d}$ depicted with the black line. However, as will be shown later after a number of cycles the motion is stabilized.

The gas pressure gradient can be reduced with a longer bearing length and consequently the gas flow rate, according to equation 6.18 . Figure $6.10 \mathrm{~b}$ shows the results of instantaneous volumetric gas leak for the stable cases $\mathrm{A}$ and $\mathrm{B}$ and the piston C. As can be seen in all cases the gas leak is mainly dominated by the piston 
velocity. The effect of the gas pressure is also visible by the negative gas leak value at TDC $(t=0.02 \mathrm{sec})$. From the results of gas film thickness variation, figure $6.10 \mathrm{a}$, we can see that for the piston $\mathrm{C}$, the minimum thickness changes as much as $50 \%$ unlike the other two stable cases A and B, approximately $25 \%$ only. The relatively bigger eccentricity influences the gas leak. This can be observed by the small increase in maximum gas flow between the two cycles. Lower gas leak in case of the piston B compared to the piston A which is yet lower than the $\mathrm{C}$ piston can be explained by the difference in sealing/sliding piston length. Apparently the more unstable motion for the piston $\mathrm{B}$ has a lower effect on the volumetric gas leak.

\section{Piston center of mass}

a)

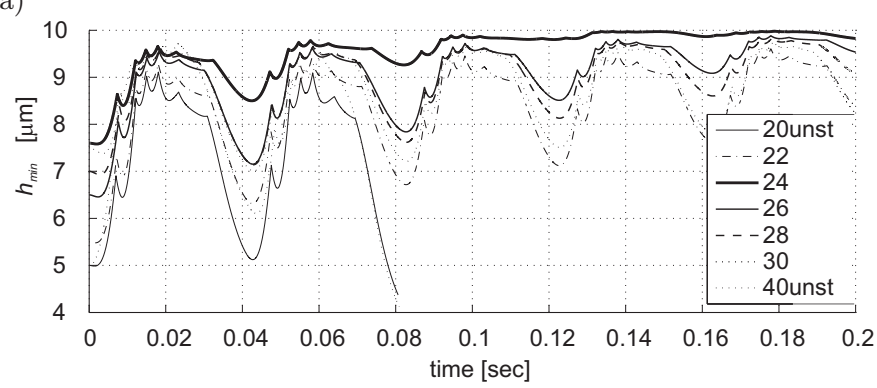

b)

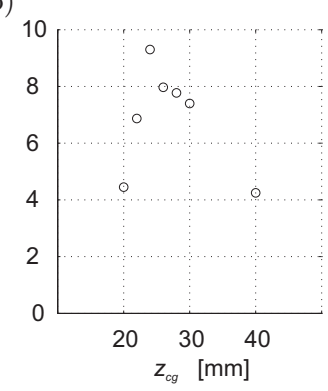

Figure 6.11: Variation of minimum film thickness with the location of center of mass a) and the logarithmic decrement b)

The stability of piston B was analyzed varying the location of the piston center of mass, figure 6.11a shows the results. It is clear this design parameter has a large influence on the piston motion. A logarithmic decrement for two first cycles is estimated, see figure $6.11 \mathrm{~b}$. The optimum location of the piston center of mass is found at $z_{\mathrm{cg}}=0.024 \mathrm{~m}$.

\section{Initial clearance}

The piston/cylinder clearance is a parameter that influences both the piston motion and gas leakage. A study of the effects on piston motion of varying the clearance will give valuable information on the possible effects of skirt deformation as this deformation influence the gas film thickness. In contrast to the previous analysis, here the piston initial position is: radial displacement, $u_{x}=3 \mu \mathrm{m}$ and rotation, $u_{x x}$ $=1.510^{-5} \mathrm{rad}$. This was done to enhance the difference in gas flow between cycles. Figure $6.12 \mathrm{a}$ shows the variations in radial motion with the clearance. Reducing the clearance leads to significantly higher gas pressure in the film. The gas pressure increase is in inverse proportion to the separation of the sliding surfaces as defined by the Reynolds equation 6.7. The higher gas pressure in the film has a positive effect 

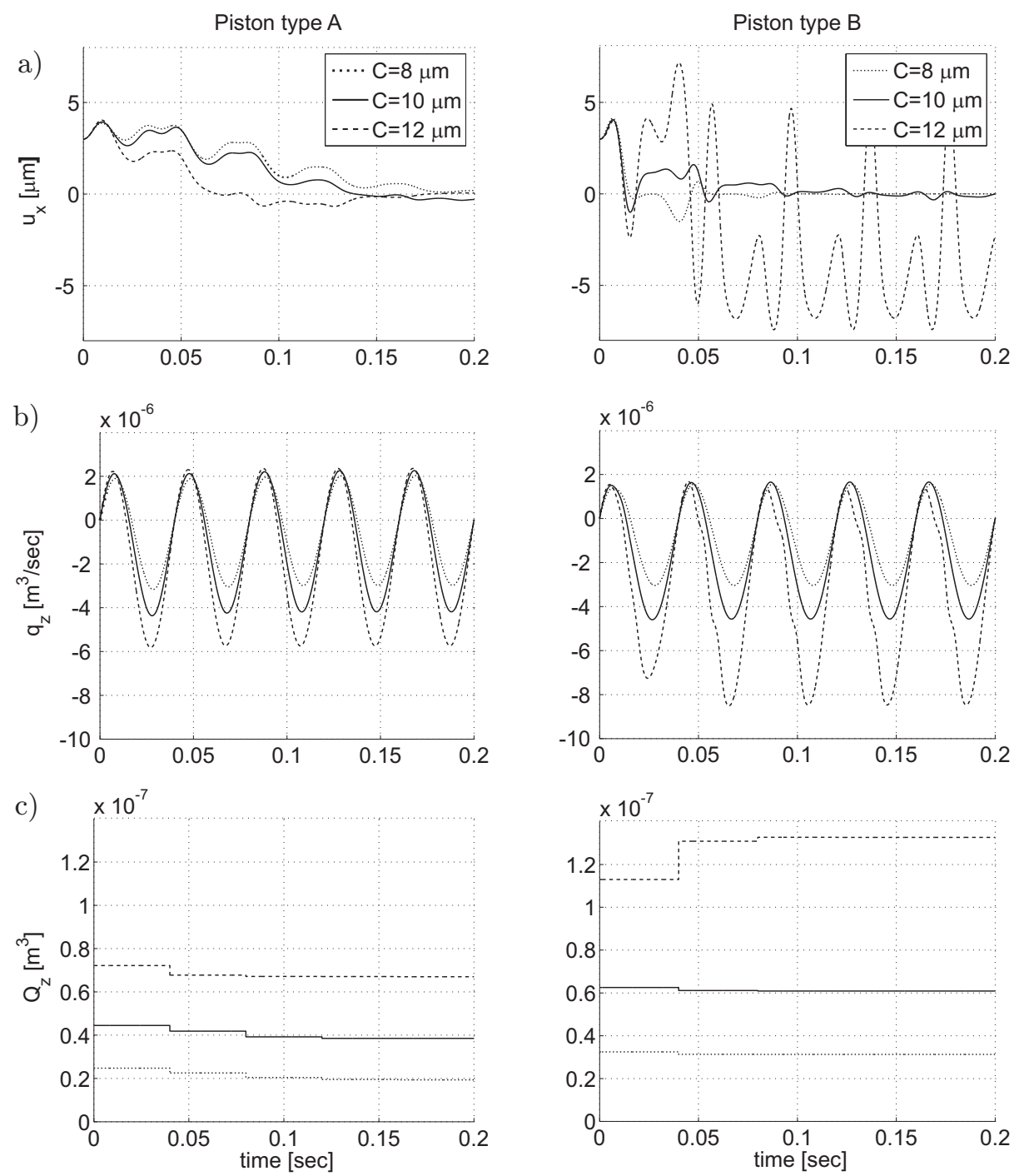

Figure 6.12: Results of SPM analysis for piston design A and B; a) variation of radial motion, b) volumetric gas flow and c) gas leakage with different initial piston clearance

on the piston B stability. Reduced film thickness leads to stable motion after two cycles already. From the other side, if the film thickness is increased to $c=12 \mu \mathrm{m}$ the piston motion would be on the edge of stability. However, for the piston A the effect is inverse, the piston centers its position, with some overshot, within two cycles for larger initial clearance. Reduced clearance would require more time to stabilize the piston motion. 

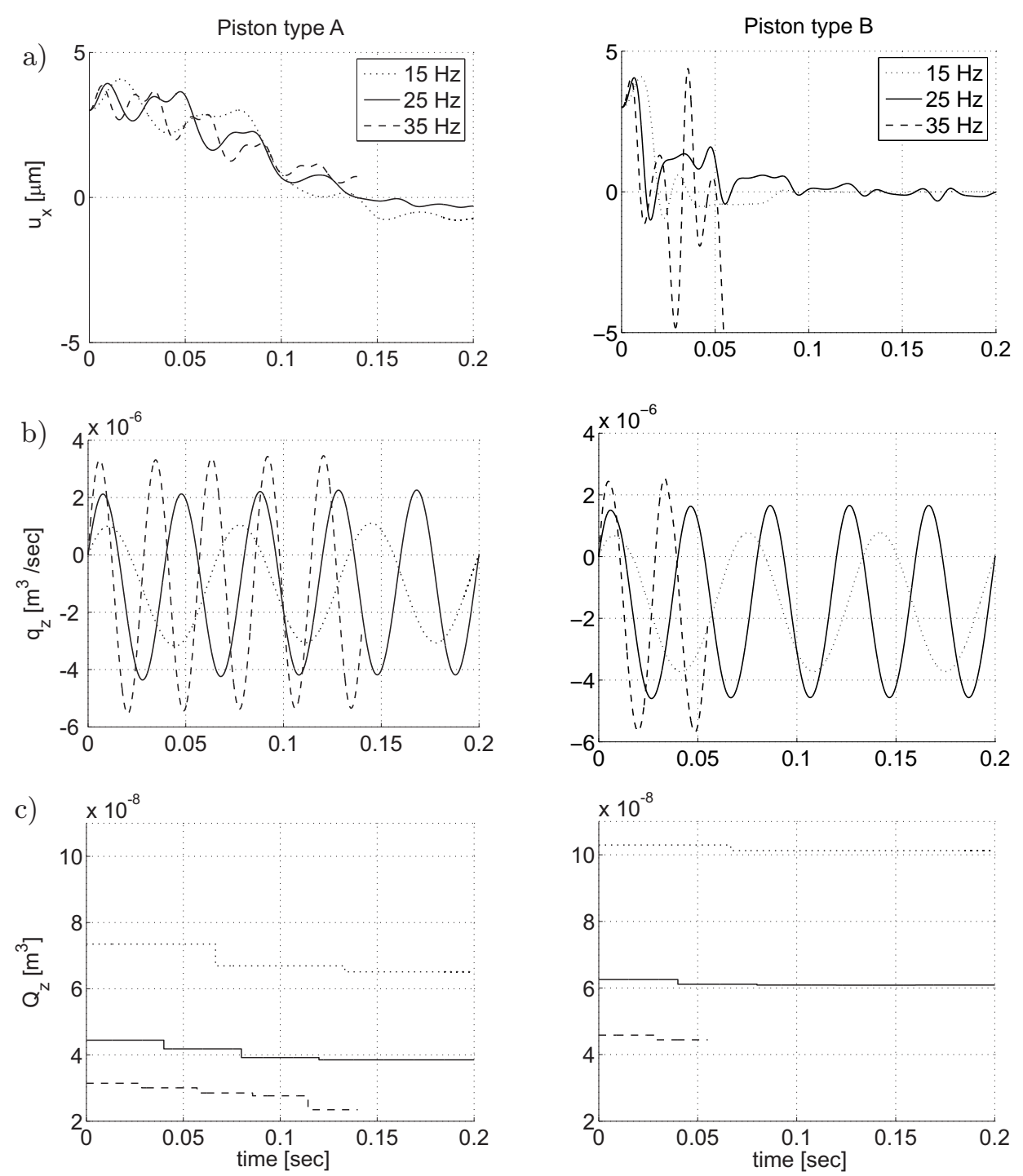

Figure 6.13: Results of SPM analysis for piston design A and B; a) variation of radial motion, b) volumetric gas flow and c) gas leakage with different piston frequency

With a larger clearance, since more volume is available for the motion, the piston tends to follow the same pattern but with a higher amplitude. Furthermore, large nominal clearance implies a considerably higher gas leakage since the gas leakage is proportional to the third power of the clearance. 


\section{Piston speed}

An analysis of the effect of the piston speed was performed by using the same base line operating conditions as described before and varying the operating frequency; 15, 25 and $35 \mathrm{~Hz}$. This corresponds to maximum piston velocities, respectively; 2.45 , 4.08 and $5.72 \mathrm{~m} / \mathrm{sec}$. Figure $6.13 \mathrm{a}$ shows the variation in radial displacement with the chosen operating frequency. As can be seen for piston A the radial motion is independent from the frequency. The higher magnitude of motion in case of piston $\mathrm{B}$ under $35 \mathrm{~Hz}$ is due to the higher forces acting on the piston skirt which is a direct response of the aerodynamic effect. Under those conditions the forces in case of the piston $\mathrm{B}, 35 \mathrm{~Hz}$ led to unstable motion. Gas flow is illustrated in $6.13 \mathrm{~b}$; as can be seen, it is also dominated by the piston speed.

\subsection{Concluding remarks}

Gas lubrication has been considered in this chapter as a possible solution for the lubrication problem in oil-free piston compressors. Low or zero wear rate giving long lifetime and low friction giving low power losses and cool running characteristics are the main advantages. This can be achieved by a higher price of the bearing components. Since in gas lubricated bearings the separated surfaces operate at a very small distance, gas bearings need high surface accuracy and strict manufacturing tolerances which requires expensive machining. Nevertheless, for already expensive machines such as Stirling coolers, higher costs for machining can be overcome.

A numerical model was presented for the analysis of the secondary piston motion. The model incorporates equations for both piston dynamics and gas lubrication. The aerodynamic forces acting on the piston are calculated by using FDM. A NewtonRaphson procedure is employed in solving the nonlinear Reynolds equations. The equations of motion are then solved using the Runge-Kutta procedure. In order to provide some validation for the model and to illustrate its usefulness in aerodynamic lubricated piston analysis, a series of parametric studies was performed on various piston design aspects.

The results explored the effects of the piston shape, initial piston/cylinder clearance, operating piston frequency on the stability. It was shown that a stable piston trajectory can be obtained if the piston skirt is properly designed. That is, the piston is non-cylindrical with both ends tapered. The piston center of mass also plays an important role and the results show that the location at $0.024 \mathrm{~m}$ from the bottom (as illustrated in figure 6.5) is favorable considering stability unlike the designed location at $0.04 \mathrm{~m}$. It was also found that the operating conditions and initial radial piston clearance considerably influence piston stability and gas leakage. The gas leakage can be reduced with longer bearing length; this means the length of the tapered section must be short. 


\section{7 \\ Conclusions and recommendations}

Piston compressors are critical components in various installations. Refrigeration systems are specific examples. Because of their application, they must provide reliable service with sufficient lifetime. Moreover, due to economical and environmental reasons high efficiency level and no harmful refrigerants are expected.

The objective of the research project was to provide design guidelines for novel design of an oil free piston compressor. This is motivated by the insufficient lifetime and reliability of the existing compressors to be used in the future applications. Design of a piston compressor is a difficult process involving analysis of complex heat transfer phenomena, thermal and mechanical interaction, tribology and material selection. For those reasons numerical and experimental tools were developed. With the help of those tools one can predict response of the trial design and/or performance of the selected materials under various operating conditions.

The additional benefit is that such a compressor would require less machine parts, like oil pumps, filters, rings etc. The consequence of this will be that the parts need to be produced in such a way that friction, thermal expansion and thermal fatigue do not cause problems in the piston cylinder contact.

Two design strategies are proposed. The piston is considered to operate either in the boundary lubrication (BL) regime or the full film lubrication (FFL) regime each with a characteristic level of the coefficient of friction, volumetric wear coefficient and surface separation, as depicted in figure 1.2. In FFL regime the sliding surfaces are completely separated by a gas film. The CoF results only from shear of the gas and surface wear is avoided. When the piston operates in BL regime physical contact between the sliding surfaces occur. This results in higher CoF and material wear depending on the surface quality. The relative sliding velocity generates heat on the interface which contribute to the material thermal expansion. If not properly designed it may lead to thermo-mechanical instability. Possible design solutions were 
investigated considering both lubrication regimes.

It should be mentioned that the problem of piston compressor design is a generic problem. The presented design approach is not limited to refrigeration systems only. In specific applications where lubricants are not allowed, like the process industry and in medical applications the same design strategy can be adopted.

\section{Regarding the experimental work}

Reliability and efficiency of the piston compressor depends mainly on friction and wear between the matching parts (assuming that dry sliding is unavoidable). Therefore coatings of low coefficient of friction were studied. Tribological tests under conditions as expected in a Stirling cooler were performed. From the results it follows that the reciprocating piston sliding conditions can be improved drastically when the sliding surface is protected with DLC coating. As illustrated the DLC coatings can provide unique properties. In particular, the combination of excellent wear resistance and low friction makes these coatings ideal for severe tribological applications. The tribological behavior of single and multi-layered a-C:H DLC coatings have been investigated in a controlled environment using the newly developed vacuum tribotester. Exceptionally low coefficient of friction $(\mu=0.01)$ and wear rate in the order of $10^{-10}$ can be achieved. The exceptional tribological behavior can be explained by the specific mechanical properties and favorable environment. The viscoplastic-like properties and structure relaxation of those materials permits weaker interaction between asperities on the sliding surfaces. On the other hand, the hydrogen-terminated carbon surface provides no chemical or physical interactions during sliding. Moreover, the formation of transferred material on the uncoated surface is paramount to achieve low friction if only one surface is coated. The presence of transferred material on disc surfaces was also observed in the performed experiments.

The gas spring test rig was designed and built to simulate operating conditions as in a piston compressor and to validate novel design aspects experimentally. The test rig is equipped with the gas installation and various sensors to accurately monitor the working conditions. Helium or other gas can be supplied to the system under controlled pressure. The fast response pressure and temperature sensors allows to investigate experimentally the complex transient heat transfer phenomena.

\section{Regarding the numerical modeling}

Modeling tools were developed to predict structural and thermal behavior of close tolerance, dry sliding piston cylinder assembly. The FEM+ALE model implemented in the code DiekA was used. The specific formulation used intensively in forming processes proved to be useful also in modeling heat transfer and thermo-mechanical distortions in a piston cylinder assembly. The ALE method allows to exclude contact search algorithms during the solution process. This offers advantages in respect 
to computational time. The model was outlined in Chapter 4. In Chapter 5 this model was applied to simulate thermo-mechanical instabilities of a conventional piston design. An alternative piston design, so-called U-piston, was also investigated. From the numerical results it follows that with the U-piston the contact pressures can be significantly reduced and under the applied simulated operating conditions will lead to stable operation. The influence of operating conditions and different materials for the piston were investigated. The best results were obtained with aluminium alloy. This was expected because of the favorable thermal and mechanical properties.

In Chapter 6 full film lubrication (FFL) has been considered as a possible solution for the lubrication problem in oil-free piston compressors. Numerical model for secondary piston motion analysis was presented. The model incorporates equations for both gas lubrication and piston dynamics. The aerodynamic forces acting on the piston are calculated by using FDM. The complete piston trajectories as functions of time were calculated with the developed computer program. The results explored the effect of the piston design on the stability. It was shown that a stable piston trajectory can be obtained if the piston skirt is properly designed. The piston is non-cylindrical with both ends tapered.

\section{Recommendations}

Although some of the friction and wear experiments have shown excellent results, reliability of the coatings performance must be improved in order to make them competitive to conventionally used coatings in piston compressors. The failure mechanism should be studied more thoroughly. Dedicated series of experiments should be conducted under the same conditions to better estimate the reproducibility. Microscope inspection of the sliding surfaces is recommended also before the experiments. Surface scratches and any signs of coating defects must be avoided. One should also consider the counter surface roughness. For those sliding surfaces where material transfer occurs, i.e. polymer coatings, there is an optimum roughness level that helps to preserve the transferred material.

It should be emphasized here that DLC stands for an extensive group of coatings. Each deposition technique offers a large number of possibilities. In addition, incorporation of additional metallic and/or gaseous elements and combinations of PECVD and PVD techniques in one deposition process means that the number of possibilities appears almost infinite. In the case of such complex materials, careful optimization towards the specific application is recommended. Further research on this subject will be very helpful and valuable in the field of tribology and machine design. The surface wear is a common problem for machine lifetime. The number of other practical applications where DLC coatings can be applied is extensive.

Considering our design problem, the coatings should also be investigated under more representative conditions, i.e. conforming surfaces, reciprocating sliding and helium environment. Those tests should be conducted on a piston compressor or an experimental set-up like the newly developed gas spring test rig. 
The numerical results have shown that the U-piston design has its advantages and it should be explored in more detail. The response under different operating conditions and sensitivity of various design parameters can be analyzed with the FEM+ALE model. Further optimization of the U-piston is recommended.

Gas lubrication technology proved to be a reliable design solution in many applications particularly those that require long lifetime. With the numerical model we have shown that a piston can center its position in time when subjected to operating conditions. In a real system the piston will be driven by a crank shaft or magnetic field. This will introduce certain lateral forces depending on the particular design. Those forces should also be taken into account. Moreover, it would be interesting to analyze the piston dynamics in a vibrating system. Those conditions will lead to occasional sliding contact. Implementation of sliding contact in the algorithm is essential for those simulations. 


\section{A \\ Oil-free piston compressors in cryocoolers}

The use of refrigeration equipment to produce temperatures lower than $77 \mathrm{~K}$ is known as cryogenics. Throughout its history several motives can be distinguished that drove the development of low temperature technology. The first relates to pure science and the development of technology to reach the lowest possible temperature. The other motive was the application of the low temperature for studying material properties. The next motive is the application of cryogenic cooling.

Nowadays cryogenics plays an important role in modern science and industry. Air separation plants use cryogenics to break down air into components for industrial and medical uses. Magnetic resonance imaging (MRI) systems that use superconducting magnets cooled by liquid helium have become common in modern hospitals. The particle accelerators used at the European CERN institute use many superconducting magnets to study the fundamental law of matters. Currently the most important application is cooling of infrared sensors. The superconductivity technology is expected to expand in the near future. The High Temperature Super-conducting (HTS) materials ${ }^{1}$ discovered in 1986 offer extra electric capacity and efficiency, but their utilization depends on reliable, efficient, low cost cooling down to cryogenic temperature. For large market applications the cryogenic system still needs to be realized in such a way that it is reliable and totally transparent to the user [80], analogous to e.g. a power supply in a PC [53]. The cryocooler must be considered as a black box and the user should not be concerned about it. This especially becomes important in space or military applications. Because of their constructional complexity cryocoolers are rather difficult to be manufactured. This aspect directly translates into higher costs.

\footnotetext{
${ }^{1}$ Conventional metallic superconductors such as lead or niobium operate below $10 \mathrm{~K}$, the relatively new ceramic superconductors such as $\mathrm{YBaCuO}$ operate typically below $80 \mathrm{~K}$.
} 


\section{Reliability and lifetime}

The term 'lifetime' is frequently used by compressor producers as the period of time that the compressor is guaranteed to operate according to the specifications. This lifetime does not mean that the compressor will not fail during the specified period of time; the cooler will have a finite probability of failing. Reliability refers to that failure probability [79]. The rate at which devices under test fail is denoted by the

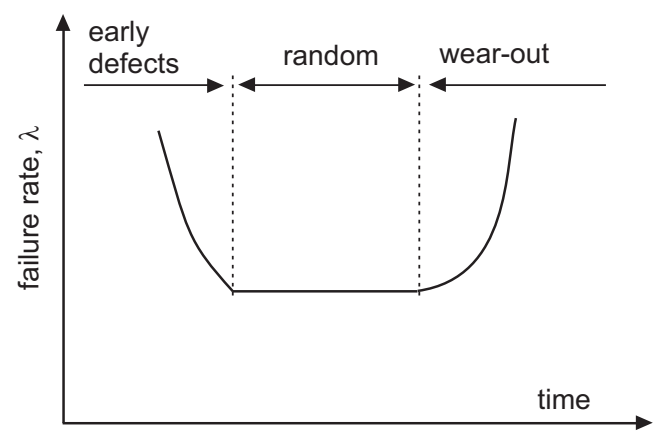

Figure A.1: Typical failure rate curve

failure rate $\lambda(t)$ : the number of failures per unit of time, normalized to the number of devices that are still operating at time $t$. A typical failure rate curve is given in figure A.1, which due to its shape is often called the 'bathtub curve'. An initial period of running-in is followed by a steady low wear. Some early defects, causing 'infant mortality', that can arise during manufacturing can be detected and removed from shipment before some predetermined number of operating hours. After shipment, the coolers are expected to operate without problems. In practice, some of them will fail because of random events (e.g. electrical damage). These events can occur randomly any time, and as a result the failure rate is constant in this period. At some point in time, wear-out mechanisms will become significant and more and more important as time evolves. In contrast to random events, material wear will affect all compressors, e.g. wear of seals or leakage of working medium. The end-of-life occurs when material wear starts to dominate over the random defects. Usually at that point, the failure rate rapidly increases. Thus lifetime is determined by material wear mechanisms and not by the random defects. 


\section{Closed thermodynamic cycles and refrigeration systems}

In a closed thermodynamical cycle a fixed mass of gas undergoes repetitively thermal processes. First, a general heat engine is schematically depicted in figure B.1a. While heat, $Q_{H}$ flows from a hot reservoir at the high temperature $T_{H}$ into a thermodynamic system, energy can be absorbed and the system is able to perform mechanical work $W$ on the environment. The efficiency of a heat engine is defined as the ratio of the work output to the heat input at the hot end: $\eta=W / Q_{H}$. Maximum performance is obtained when all thermodynamic processes involved are reversible i.e. with no losses and, therefore, the entropy $S$ of the thermodynamic system should not change. Among other sources of losses: sliding friction and heat transfer can be mentioned here. A typical example of ideal cycle is the Carnot cycle. It consists of four processes, two isothermal and two adiabatic, depicted in figure B.1d. For analysis purposes the thermal cycles are outlined in diagrams e.g. pressure-volume (p-V) or entropytemperature (T-S) where the individual thermal processes are represented by lines. The work performed on the environment can be expressed in differential form as $d W=P d V$. Therefore the net work achieved equals the area enclosed by the curves in the P-V diagram. Similarly, because $d Q=T d S$, the first law shows that the work supplied also equals the area enclosed by the curves in the T-S diagram: $\left(T_{H}-T_{A}\right) \mathrm{dS}$. Therefore the engine Carnot efficiency is given as:

$$
\eta_{\text {Carnot }}=\frac{W}{Q_{H}}=\frac{T_{H}-T_{A}}{T_{H}}
$$

The Stirling cycle is another example of ideal cycle. Common for the two cycles is that the entropy of the working gas can be changed significently at constant temperature, while absorbing or rejecting heat. When the thermodynamic system in a heat engine is reversed, a heat pump or a cooler can be realized. 
a)

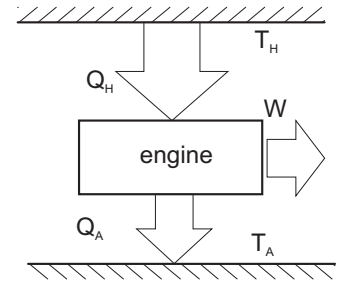

b)

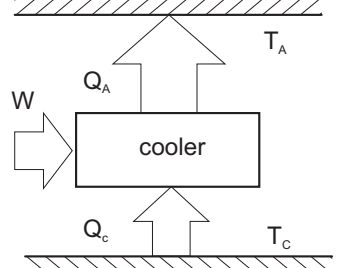

c)

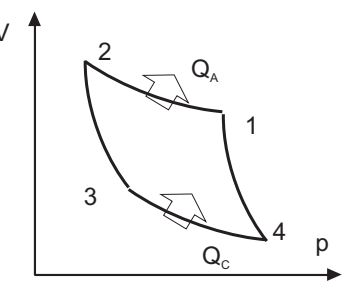

d)

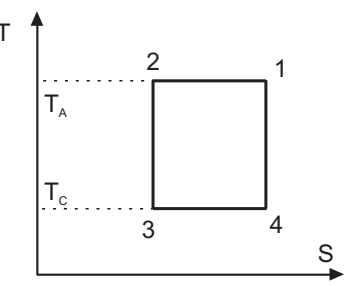

Figure B.1: Schematic representation of: a) heat engine, b) cooler, c) p-V and d) T-S diagram of the Carnot cycle

\section{Refrigeration effect}

In the reverse case, when the heat engine works as a cooler heat is removed from a lower temperature reservoir $Q_{C}$ and rejected to an ambient temperature reservoir $Q_{A}$ by the input of mechanical work $W$. This is schematically shown in figure B.1b. The refrigeration effect is obtained via mechanical work performed on a working gas inside a compression space according to the specific thermodynamical cycle. In this work we will consider only the reversed Stirling and Rankine cycle. In those cycles a piston compressor plays an essential role converting the mechanical energy into thermal. The piston compressor generates a pressure wave, see e.g. figure B.2. The magnitude of the generated pressure directly determines the cooling performance.

In the analysis of a refrigeration cycle the amount of work, $W$ required to remove a given amount of heat $Q_{C}$ is considered. The overall performance of a refrigeration system is usually expressed as the coefficient of performance (COP) that is defined as the ratio of the heat removed from the low temperature reservoir, $Q_{C}$ to the required amount of mechanical work, $W$.

$$
C O P=\frac{Q_{C}}{W}
$$

Analogous to the heat engine Carnot efficiency, the COP is limited by Carnot's COP. A reversed Carnot cycle represents the most efficient possible refrigeration cycle operating between two specific temperature limits.

$$
C O P_{\text {Carnot }}=\frac{T_{C}}{T_{A}-T_{C}}
$$


where $T_{C}$ is the temperature of low temperature reservoir. Frequently, efficiencies are given as a percentage of the Carnot efficiency.

\section{Stirling cycle}

Robert Stirling invented a simple hot-air engine converting heat into mechanical power and patented it in 1816. Compared to its contemporary, the steam engine, the Stirling engine worked with a much lower operating pressure and, therefore, had a much lower risk of explosion. In 1834 Hershel suggested to reverse the operation of the Stirling engine and thus turn it into a cooler. Many people tried to put the Stirling cycle to work for refrigeration. The efficiency of these early machines, however, was rather unsatisfactory. The turning point came during the second world war. At that time Rinia and his colleagues at Philips Research Laboratories/the Netherlands reconsidered the Stirling cycle as a hot-air engine to power radio sets at remote locations. In 1945 Rinia and Du Pré converted a one-horse-power engine into a refrigerator and obtained a temperature of $83 \mathrm{~K}$ [54].

Essential elements of a Stirling device are two spaces: compression (CS) and expansion (ES), at different temperatures, whose volumes can be varied cyclically and which are connected by ducts containing heat exchangers and a regenerator. These elements can be combined in a wide range of mechanical configurations. The $\beta$-Stirling configuration is most common in cryocoolers and it will be used for illustration of the Stirling cycle. In the ideal Stirling cycle a working gas is alternatively compressed and decompressed. The change in gas pressure will subsequently lead to change in temperature. This is accomplished by moving the gas back and forth between cold and hot heat exchangers with a regenerator in between. In the Stirling device heat is absorbed at the cold head $(\mathrm{CH})$ due to an expansion process and rejected at the warm end due to compression process.

The piston and displacer can move up and down independently, see figure B.2. In the $1^{\text {st }}$ process, the working gas is compressed isothermally between piston $(\mathrm{P})$ and displacer (D). Compression heat is created in the compression space and rejected to the ambient reservoir, $Q_{A}$. Next in an isochoric process, the gas is displaced through the regenerator $(\mathrm{R})$ in a constant volume by simultaneous movement of both piston and displacer. The compression heat is stored in the regenerator. The gas temperature is cooled to almost the temperature prevailing in the expansion space. The $3^{r d}$ process is an isothermal expansion. The displacer together with the piston is driving downwards. The working gas expands absorbing heat from the cold environment $Q_{C}$. Actual cold production takes place here. The last $4^{\text {th }}$ process is an isochoric displacement. The displacer is driving upwards. Gas returns to the space between the displacer and piston. Passing through the regenerator the amount of heat stored in the regenerator is transferred back from the regenerator to the gas. Stirling cryocoolers are able to lift heat down to $77 \mathrm{~K}\left(-200{ }^{\circ} \mathrm{C}\right)$, which is sufficient to liquefy air. Lower temperatures down to $40-60 \mathrm{~K}$ can be obtained depending on the particular design. Stirling cryocoolers for this purpose are competitive with other cryocooler technologies. The working gas should have a low heat capacity, so that a 

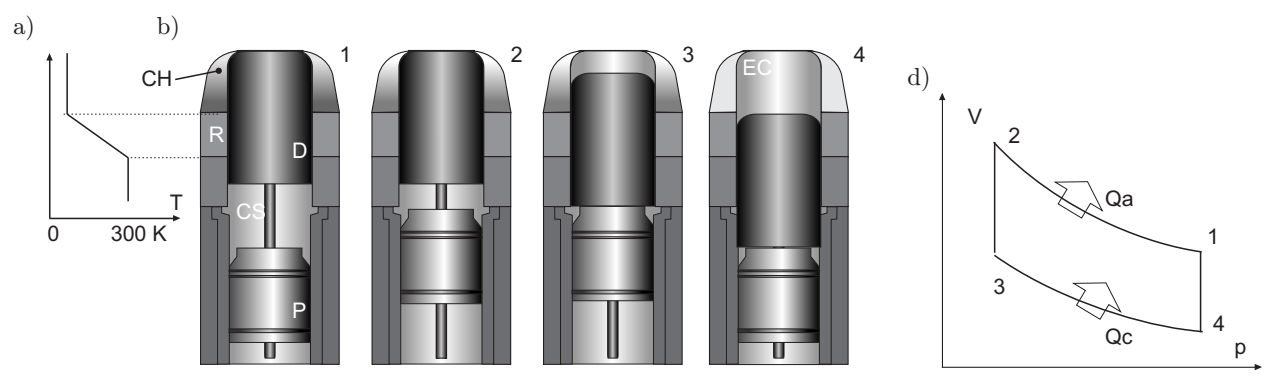

c)
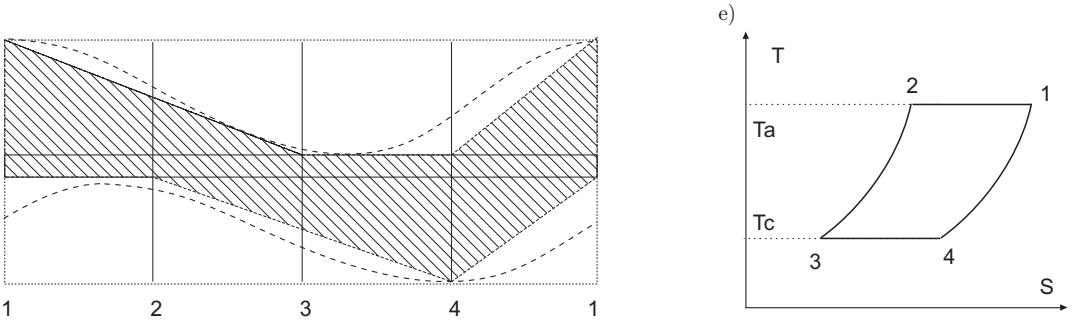

Figure B.2: Model of a $\beta$-Stirling cooler a) temperature profile along the heat exchangers b) four steps of the Stirling cycle c) discontinuous and harmonic displacement-time diagrams of the two pistons (the shaded area is proportional to the volume occupied by the working gas) d) p-V and e) T-S diagram of the Stirling cycle

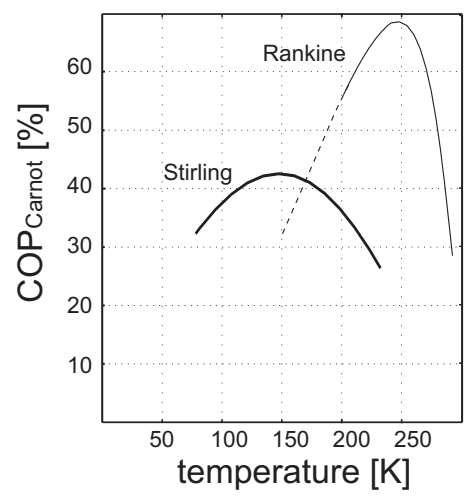

Figure B.3: Efficiency of Stirling and Rankine coolers

given amount of transferred heat leads to a large increase in pressure. Considering this issue, helium would be the best gas because of its very low heat capacity. On the other hand low viscosity and high thermal conductivity of hydrogen makes the gas a better choice. However, most technically advanced Stirling devices use helium as the working gas, because it functions close to the efficiency and power density of hydrogen with fewer of the material compatibility issues. Helium is inert, which 


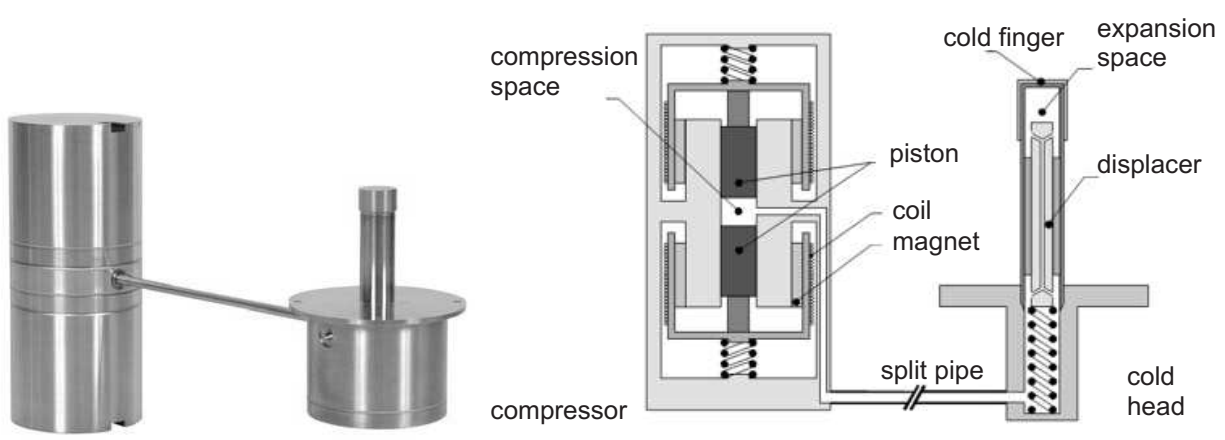

Figure B.4: Stirling cooler, split configuration, Thales-Cryogenics/Eindhoven

removes all risk of flammability, both real and perceived. Regarding friction and wear of sliding components the environment plays a dominant role. Therefore the choice of the working gas may have a considerable effect on the compressor's lifetime.

\section{Stirling cryocoolers}

Stirling cryocoolers are produced as crank driven or free moving piston. The lifetime and reliability of small size cryocoolers has been improved significantly over the last years mainly due to the improvements in the compressor. The requirements were originally put forward by space applications but later on where also demanded by commercial applications of high temperature superconductors. Incorporation of flexure bearings and clearance seal in the free moving piston allowed to eliminate rubbing contact and material wear which is the limiting factor in compressor lifetime. With this technology the specified lifetime of these compressors can exceed $50.000 \mathrm{~h}$.

The free piston compressor technology allows designing efficient and compact cooling units. Indeed, free piston Stirling coolers driven by an electric linear alternator have proved to be reliable devices. However, this is obtained at the cost of compressor total mass. Free piston compressors are approximately three times as heavy as crank driven compressors. For the applications where the ratio weight to power matters, crank driven compressors are preferred. However, the lifetime of those compressors is limited due to material wear.

A wide variety of smaller size Stirling cryocoolers are commercially available for tasks such as the cooling of electronic devices. For these applications cryocoolers based on Stirling cycle are the highest performance technology available to extract heat efficiently down to cryogenic temperatures. Thales - Cryogenic BV/Eindhoven offers cryocoolers in an integrated and split version. In the integrated version the crank driven piston compressor and the cold finger are part of the same body[12]. This provides high efficiency and allows smaller dimensions, lower weight and lower input power. In the split version, the compressor and cold finger are separated components 
linked by a split pipe. This design provides flexibility and isolates the cooled object from the (vibrating) compressor.

For large cryocoolers used for gas liquefaction plants, crank driven compressors are used. Stirling Cryogenics BV produces fully automatic, stand-alone, liquid gas production plants and cold cabinets for cryogenic treatment of metal components. Stirling BV offers also solutions for the HTS market to cool different applications and devices. The so-called Power Cooler provides refrigeration down to $65 \mathrm{~K}$ or, in the case of the two-step cooler, down to $20 \mathrm{~K}$. The specified lifetime is typically 5,000 hours. The figure B.5 shows a cryogenerator with the trade name SPC-1.
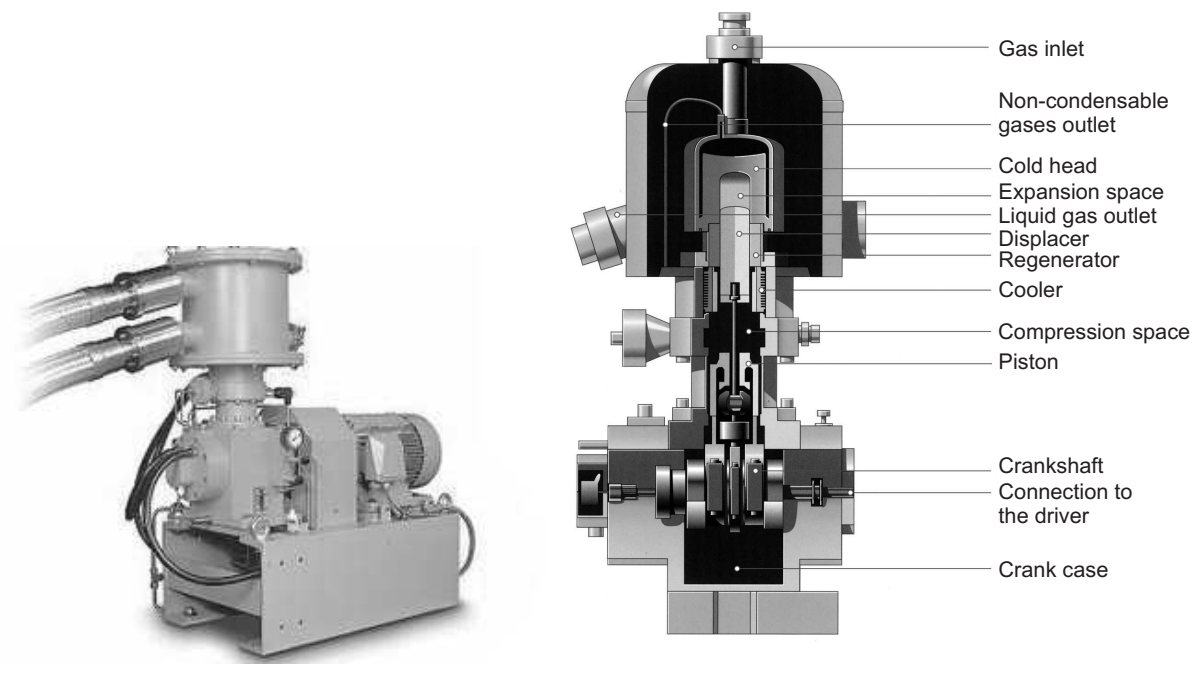

Figure B.5: Cryogenerator SPC-1, Stirling Cryogenics BV/Eindhoven

\section{Rankine cycle}

A schematic diagram of a typical Rankine cycle, also called vapor compression cycle, is given in figure B.6a. The refrigerant in a gaseous form is isentropically compressed by the compressor and delivered to the high pressure condenser (step 1-2). The temperature of the refrigerant increases during this process well above the temperature of the surrounding medium. The refrigerant enters the condenser as a superheated vapor, point 2. Inside the condenser the refrigerant is cooled and heat is transferred to ambient temperature reservoir (step 2-3). Because of the heat transfer the output of the condenser is a saturated liquid, point 3. Next the refrigerant is expanded with a throttling valve to a lower pressure and sent to the evaporator, point 4 . Due to the expansion, the temperature of the refrigerant drops below the temperature of the refrigerated space. During step 3-4 the actual refrigeration process take place, heat from the low temperature reservoir is absorbed. The gas from the evaporator leaves 
as saturated vapor and re-enters the compressor, completing the cycle. The steps of the thermodynamic cycle are also illustrated in the T-S diagram in the figure B.6b.

a)

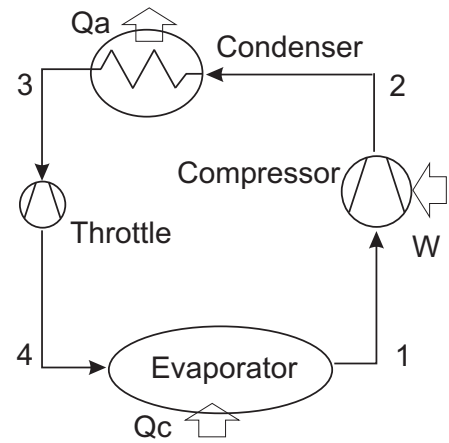

b)

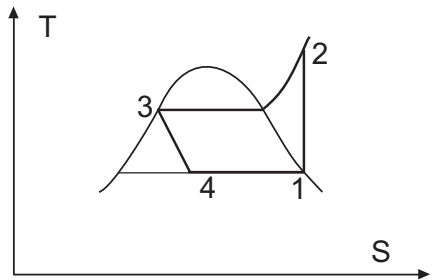

Figure B.6: a) Schematic diagram of the Rankine cycle and b) T-S diagram

The heat transfer in the condenser takes place mainly as a result of a phase change, compared to the isothermal heating of an ideal gas in the Carnot cycle. This effect enhances the efficiency being closer to the Carnot efficiency. The Rankine cycle is a common alternative to the ideal Carnot cycle. In contrast to the Carnot or Stirling cycle, in the Rankine cycle the working fluid, also called the refrigerant, changes phase between liquid and gas. The refrigerant is chosen based on its boiling point and heat of vaporization. The physical properties of the fluid limit the operating temperature. A relatively high COP in a one stage Rankine device can be reached down to 200-340 $\mathrm{K}$, see figure B.3. The dotted line represents the COP for a two stage Rankine cycle. Below that range there are no suitable refrigerants with such low boiling points.

\section{Rankine systems}

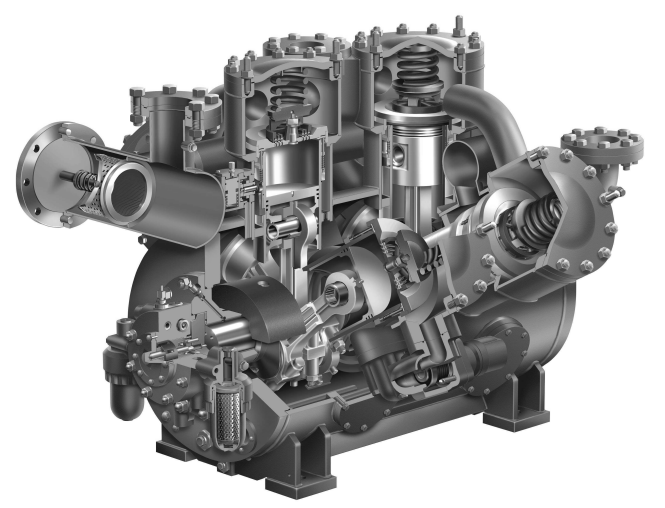

Figure B.7: Refrigeration compressor, Grasso 
Crank driven piston compressors are the most widely used configuration in Rankine systems, see figure B.7. These systems are suitable for working under high pressure and large capacity. Due to the high contact forces on the piston/cylinder interface, the sliding system requires oil lubrication.

For environmental reasons the harmful refrigerant R-12 was systematically phasedout from refrigeration systems. It was mainly replaced by a new range of hydrofluorocarbon refrigerants including R-134a. In recent years, the refrigeration industry has shown great interest in the use of natural refrigerants, such as $\mathrm{CO}_{2}$. With the introduction of environmentally friendly refrigerants in oil free piston compressors, the sliding interfaces may no longer be able to withstand the harsh operating conditions caused by smaller clearances and increased loads found in the compressors. Such severe conditions will lead to excessive wear and premature failures, thus becoming a major liability issue for compressor manufacturers. The transition to new refrigerants and the advent of natural refrigerants has made the search for wear resistant and low friction materials and interfaces a topic of great interest [15] [16]. 


\section{Installations and procedures}

The sketch and instrumentation diagram of the test rig is given in figure 2.4. The helium installation (depicted dotted line) supplies the gas to the test rig. The gas He4.6 is fed from a pressure bottle 200 bar. A set of tree filters (HFS) is installed on the supply line: $0.1 \mu \mathrm{m}$ element, $0.001 \mu \mathrm{m}$ element and active coal element. The pressure is reduced from the bottle pressure to the charge pressure with the pressure reduction valve $(\mathrm{RV})$.

Before starting an experiment, in order to minimize air contamination in the working gas the compression space is vacuumed. Firstly, 3-way valve (V2) is opened for the vacuum pump and closed on the helium-bottle side. The gas spring will be vacuumed up to a reasonable under-pressure $(0.1 \mathrm{bar})$, with sufficient time in order to evacuate gases above and under the compressing piston, and out of the crankcase space as well. Next, valve V2 will be closed on the vacuum side and opened for the helium charge with an overpressure of 2.5 bar. The gas spring will be charged and discharged several times in order to clean the compressing space from the air residuals and enable the compressing gas to be as clean as possible. The last step before charging will be flushing the set-up while operating on low speed. Namely, helium supply will feed the gas to the compressing space, which will, due to the over-pressure, blow by the compressing piston, through the buffer space, by the guiding lower piston and the discharge valve (DV) and out of the set-up. Finally, the DV will be closed and the gas spring will be charged to a pressure of 1.5 bar. Care will be taken to give enough time for the pressure to stabilize; the set-up will be operated then. The pressure controller will monitor the buffer space pressure between the two pistons and keep it on the mean cycle pressure. Static pressure transducer measures the pressure in the buffer space between the two pistons. The helium charge in the set-up is controlled via the line connecting the buffer space and the Helium bottle, thus SPT is giving precise information of the pressure. The chosen sensor is Druck PMP 4000 series, with Hastelloy steel membrane construction, 0-35 bar absolute measuring range, 0.04 $\%$ overall accuracy and $0-5 \mathrm{~V}$ output.

The cooling installation supplies air from the pressure line ( 6 bar) available in the 
laboratory room via one $4 \mu \mathrm{m}$ filter (AFS). The cooling air is supplied into the gas spring via a specially constructed feedthrough on the top frame. With the same feedthrough the sensors wires are conducted. The cooling air is first directed on the cylinder head. With the radially distributed cooling passages the air flows into the cooling channel along the cylinder head. Here the cylinder head outer surface temperature is monitored with the infrared sensor. The cooling channel in the cylinder head and the liner section are designed to make sure the cooling air velocities are similar. On the bottom the cooling channel is sealed from the buffer space with an O-ring between the lower flange and the liner. Further, via radial passages in the main frame the cooling air is directed with the collector out of the test rig.

Measured data is collected using two M-serie; PCI boards 6250 and 6280 (National Instruments). The devices are optimized for high accuracy at fast sampling rates. To ensure accuracy they have an onboard NI-PGIA 2 amplifier designed for fast settling times at high scanning rates, ensuring 16-bit accuracy even when measuring all channels at maximum speeds. The devices have a minimum of 16 analog inputs, 24 digital I/O lines. Sampling rate: $1.25 \mathrm{MS} / \mathrm{s}$ single channel, $1.00 \mathrm{MS} / \mathrm{s}$ multichannel.The NI PCI-6280 is a high-accuracy multifunction M Series data acquisition (DAQ) board optimized for 18-bit analog input accuracy. This resolution is equivalent to 5 digits for DC measurements. For accuracy, the NI-PGIA 2 amplifier technology is optimized for high linearity and fast settling to 18 bits, and programmable lowpass filters reject high-frequency noise and prevent aliasing. With the NI LabVIEW software a custom data acquisition application was developed. 


\section{Coating deposition techniques}

Due to intensive research on DLC coatings dictated by the large number of applications in many branches of industry, many advanced deposition methods have been developed. In $[39,72]$ an extensive review on the methods used in industry can be found. Most of the carbon coatings are obtained by chemical (CVD) or physical vapor deposition (PVD) techniques. The process takes place in a chamber with controlled atmosphere at relatively low pressure and high temperature. The coating is formed on the surface from a vapor containing the coating elements material. The major difference between them is the evaporation method of the coating materials as well as the process temperature.

PVD, the metallic coating component (for example $\mathrm{Ti}, \mathrm{Al}$ or $\mathrm{Cr}$ ) is vaporized or sputtered while the non-metallic component (e.g. C, N) is supplied in the form of a gas to the reaction chamber. Using graphite or a carbonaceous target will lead to amorphous carbon a-C:H and high $\mathrm{sp}^{3}$ content with low hydrogen content (less than $10 \%$ ) For PVD processes the temperature is typically in the range of $200-500{ }^{\circ} \mathrm{C}$.

CVD, a coating is deposited on the surface due to chemical reaction between a gas of at least two components flowing around the coated workpiece. For classical CVD processes the process temperature is high, in the order of $600-1100{ }^{\circ} \mathrm{C}$. This high temperature can be destructive for some materials due to both altering the microstructure and changing the dimensions of the component. With a plasma or laser enhanced PECVD process the temperature can be significantly lowered.

As mentioned, internal stresses as well as other properties can be improved by combining different coating materials. The composition of the coating material can be changed resulting in various structures: a) multilayer, has a better tribological performance because the internal shear stresses are distributed over many interfaces. The matching of the thermal expansion and chemical bonding of the subsequent layers also improves the coatings adhesion. b) gradient coatings are a special type of the multilayer, here the material composition varies continuously over the coating thickness, which has the advantage that there is no abrupt change in composition 
and no stress concentration at the interfaces. By a proper control of the deposition parameters it is possible to obtain a layer with diverse content of the diamond or graphite phases. Thus obtained properties result from the phase content. c) multiphase, multicomponent, composite coatings, it is possible to obtain coating of different phases and/or components. In composite coating one phase is dispersed in a continuous matrix of the other phase.

The delivered coatings, the coatings named 26A and 27A delivered by Bekaert Advanced Coatings were prepared with PACVD deposition method, same coatings as the commercially available, Dylyn $+[8]$. The coatings are characterized by multilayer structure (metallic adhesion layer, transition layer, DLC layer). According to the supplier the hydrogen content is in the range of 25-30 at \%, coating thickness 1.5 to $2 \mu \mathrm{m}$. Adhesion strength $>20 \mathrm{~N}$ critical load measured by the scratch method.

Hauzer Techno Coating BV delivered multilayer tungsten-containing DLC coatings, named H1. The coatings were produced by the PVD method. Detailed study on the influence of the metal to carbon ratio on the mechanical and tribological properties are reported in [76]. The adhesion measurements with scratch testing showed good adhesion level $\geq 50 \mathrm{~N}$ critical load. We measured thickness of the coatings, 2.3 to 2.5 $\mu \mathrm{m}$, using the Calotester.

The second coating type delivered by Hauzer is produced with PECVD process with highly hydrogenated plasma, up to $95 \% \mathrm{H}$ and $5 \% \mathrm{C}$. The coating has a wear rate from about $10^{-10}$ to $5 \times 10^{-11} \mathrm{~mm}^{3} \mathrm{~N}^{-1} \mathrm{~m}^{-1}$ measured in a dry, inert environment and coefficient of friction from 0.001 to 0.007 measured in a substantially clean nitrogen environment [22]. 


\section{Environmental effects and superlubricity of DLC coatings}

In inert or vacuum conditions, friction and wear can be accurately controlled by hydrogen. Highly hydrogenated films may exhibit superlow friction due to a rather high hydrogen concentration on the surface. The presence of hydrogen atoms reduces adhesive forces between the opposite surfaces by eliminating dangling $\sigma$-bonds on the carbon based surface. Hydrogen has a strong chemical affinity to carbon. It bonds strongly to the carbon atoms and thus effectively passivates their free $\sigma$-bonds leading to very little adhesive interactions.

Erdemir further explains possible phenomena that allow to reach super low coefficient of friction [24]. One more reason why these DLC films provide superlow friction is that, when the free electrons of hydrogen atoms pair with the dangling $\sigma$-bonds of surface carbon atoms, the electrical charge density is permanently shifted to the other side of the nucleus of the hydrogen atom and away from the surface. Such a shift in charge density allows the positively charged hydrogen proton in its nucleus to be closer to the surface than the electron that is used up by the $\sigma$-bonds of the surface carbon atoms. Therefore, it is reasonable to state that the creation of such a dipole configuration at the sliding interface should give rise to repulsion rather than attraction between the hydrogen-terminated sliding surfaces of the DLC films.

Hydrogen doesn't control exclusively the superlow friction of DLC coatings. Silicon incorporation in DLC structure may also reduce friction ( 0.007). The experiments were performed in vacuum, a steel ball was sliding on the flat surfaces coated with [77]. Fluorine incorporation in DLC may also reduce CoF to values lower then 0.01 . With an a-C:H:F coating containing 18 at. $\% \mathrm{~F}$ and only 5 at. $\% \mathrm{H}$ the stabilized CoF observed in vacuum is only about 0.005 [32]. Super low CoF was reported in 
ambient air conditions [33] in DLC coating containing sulfur. The a-C:H:S coating contains 30 at. $\% \mathrm{H}$ and only 5 at. $\% \mathrm{~S}$. Thermal stability has been proved to be $300{ }^{\circ} \mathrm{C}$ in air. Due to $\mathrm{S}$ incorporation the hardness decreased from 13 to $8 \mathrm{GPa}$. The positive nitrogen effect on sliding DLC under different conditions and environments has also been reported [74] [51]. Ultra and super low CoFs were measured.

DLC coatings are known to be thermodynamically unstable above a certain temperature. Upon exposure to elevated temperature gradual transformation may proceed from a highly disordered amorphous state to increasingly ordered or graphitic structure. If the DLC coating is hydrogenated, some of the hydrogen atoms, especially the one unbounded to carbon, may begin to diffuse out and leave a relatively porous structure behind. Under such conditions, when the surface chemistry and structure changes the $\mathrm{CoF}$ consequently will change as well. Hydrogen free coatings may have relatively higher endurance limits to elevated temperatures. The density of those films doesn't change much up to very high temperatures (i.e. $500^{\circ}$ ) at which point it may also transform to graphite. According to [28] a-H:C coatings depending on the hydrogen content may provide low friction up to about $300{ }^{\circ} \mathrm{C}$ during short duration (i.e. approx $1 \mathrm{~h}$ ) longer duration tests tend to wear faster than at room temperature. 


\section{The vacuum tribo-tester}

The friction characteristics were evaluated in a pin-on-disc configuration tribometer. The vacuum tribotester was developed in the Surface Engineering and Tribology group (University of Twente) during this project [67]. The tribometer is built in a Norcal 18" vacuum chamber. The normal load $F_{n}$ on the pin is applied by means of the Squiggle SQ115-V piezo motor (motor2, see figure F.1) driving the measurement arm via blade springs. The motor has a moving range of $15 \mathrm{~mm}$ with a maximum force $5 \mathrm{~N}$ and linear velocity 0.001 to $2 \mathrm{~mm} / \mathrm{s}$ with displacement resolution of $20 \mathrm{~nm}$. A feedback system is incorporated to increase accuracy of the motion control. The feedback is realized by a Sheavitz MHR250 LVDT (Linear Variable Differential Transformer) which measures expansion of the blade springs. The signal from the LVDT sensor is logged to an amplifier processed and corrections are made to the motion. The measurement arm is supported on elastic hinges of known stiffness. The force $F_{n}$ is determined as a function of the blade spring stiffness, length of the measurement arm as well as the stiffness of the elastic hinges.

The support table is mounted on two linear roller bearings allowing lateral motion. The position is controlled by a SQ115-C motor with a LVDT feedback. This option allows to measure $\mathrm{CoF}$ on sliding tracks of different diameters. The friction force $F_{t}$ is acquired by a Kistler 9203 piezo transducer mounted in the measurement arm. The pin's vertical position can also be measured. However this option was not used during our experiments due to insufficient accuracy.

The disc sample is driven by the Kollmorgen (H-344) brushless servomotor with a planetary gearbox 5:1 (NemaTrue34) and a controller (ROL-20012). The motor is controlled with a Galil DMC 1840 PC-card which is fully programable with LabView. In addition to a constant rotating speed, with the maximum $1 \mathrm{~m} / \mathrm{s}$, it can also be programmed for reciprocating motion. The maximum sliding speed in reciprocating motion is $0.1 \mathrm{~m} / \mathrm{s}$. The maximum sliding speeds are associated with motor (inertia) limits. The motor is placed outside the vacuum chamber. The disc holder is connected via an elastic coupling and the feedthrough in the vacuum chamber. The elastic coupling eliminates possible misalignments between the motor and the disc. 
Several feedthrough ports are used to supply measurement signals and liquids into the vacuum chamber. A rotation feedthrough, a bellow seal Pfeiffer UD 140 is used to seal the disc holder / electric motor coupling. Gases are supplied with the Pfeiffer DN $40 \mathrm{CF}-\mathrm{F}$ valve. Signals are conducted with various MPFPI ports. The vacuum chamber is connected to a turbo molecular vacuum pump Pfeiffer Turbocube 071. According to requirements an appropriate gas can be introduced to the chamber at an ambient pressure.

The data are measured via an in-house developed software. This software was written in the National Instruments LabVIEW environment to automate signal acquisition, data processing, and driving of the motors.

a)

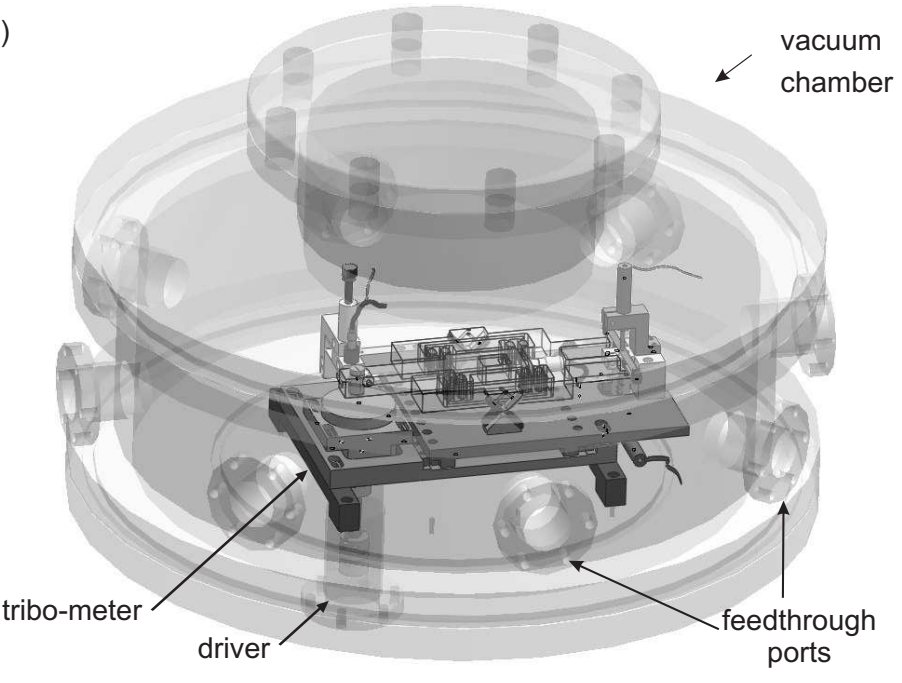

b)

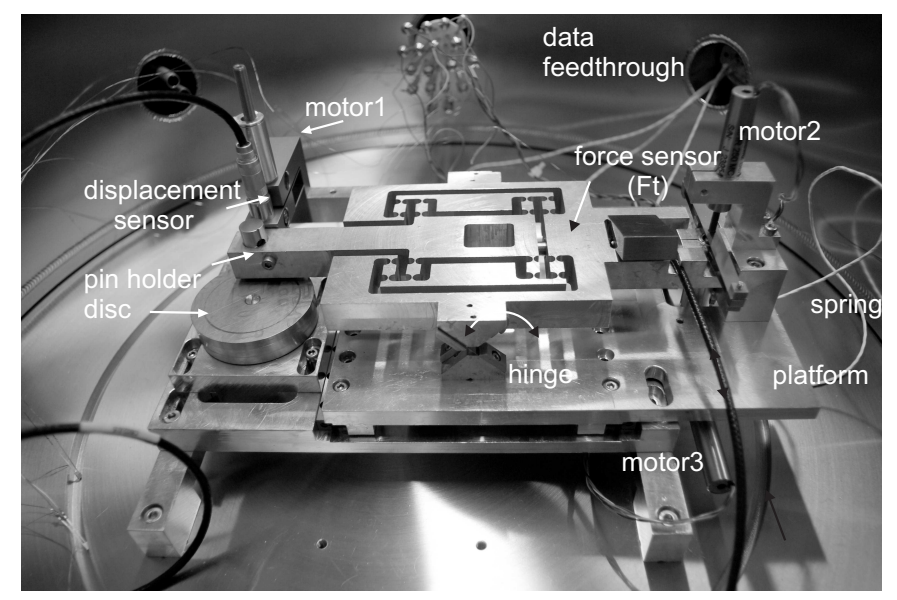

Figure F.1: a) Vacuum tribotester and b) photo of the pin-on-disc tribometer 


\section{Friction measurements performed in vacuum}

The three coatings 27A, H1 and NFC were tested in vacuum. As the counter sliding surface, the 52100 discs were used. The other test conditions are given in table 3.6.

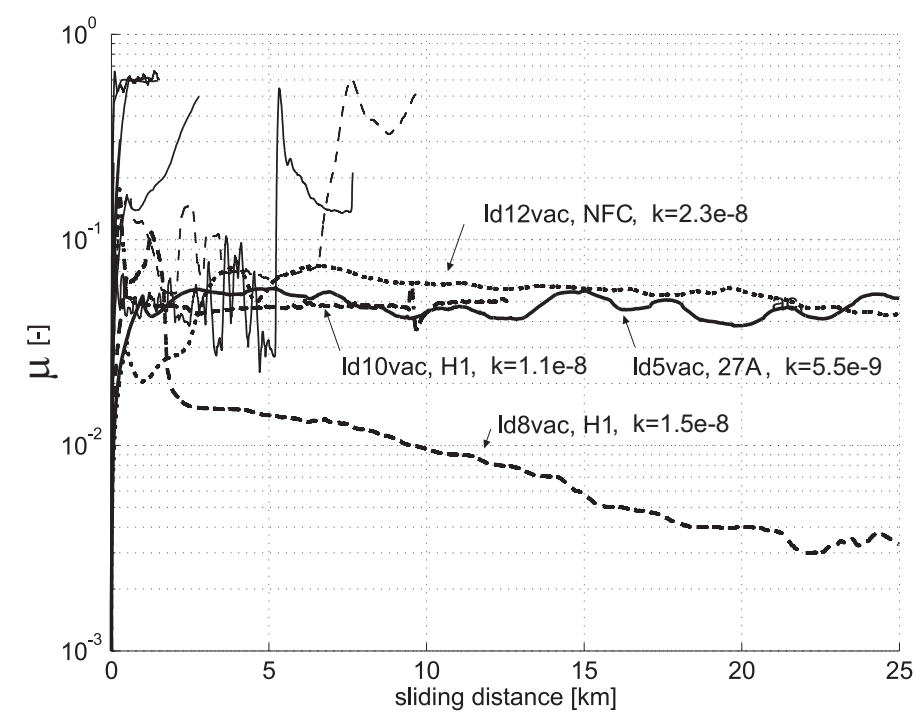

Figure G.1: CoF of different coatings measured in vacuum with 52100 discs

Figure G.1 shows all CoF measuremens performed in vacuum. Each coating type has been tested four times, twelve tests in total. As can be seen from the results, four tests, namely lt5vac, lt8vac, lt10vac and lt12vac shows that those coatings have 
a)

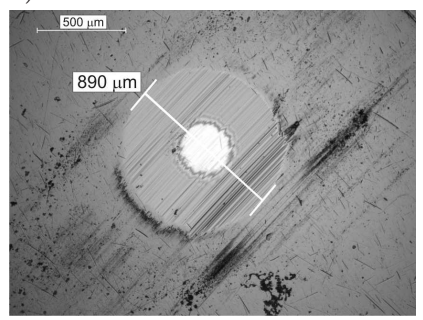

b)

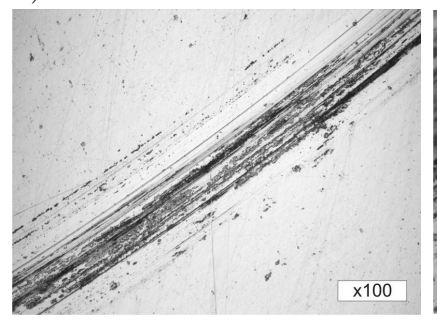

c)

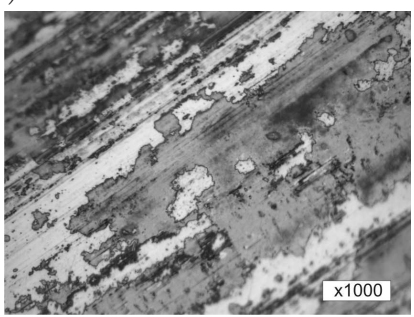

Figure G.2: Wear observations after the test ld8vac, coating H1, performed in vacuum: a) pin worn spot, b) sliding track on the 52100 disc with 100x magnification and c) zoom-in on the wear track, magnification 1000x

run-in to a state of ultra and super-low CoFs (ld8vac). The H1 coating gives again the best performance in terms of $\mathrm{CoF}$ and reproducibility. The test ld10vac was stopped due to computer problems. If coating failure occur, most often it would be during the running-in stage, very often soon after the test has been started. The same observation was made after the previous tests in helium. In vacuum however three tests, depicted with the thin line, failed after $5 \mathrm{~km}$ sliding distance. This can be explained by the poor convective heat transfer in vacuum.

The degree of material wear of selected tests was inspected with a light microscope and SEM (Scanning Electron Microscope). The test ld8vac from the figure G.1a shows the lowest CoF value among all the experiments. The worn spot of the pin is shown in figure G.2a. As can be seen there is a considerable amount of free wear debris covering sides of the worn spot. The white circle in the middle is the interlayer material as was revealed with EDX (Energy-Dispersive X-ray) measurement. Irregular and rather dense transfer material can be seen on the images G.2b and G.2c. The agglomeration of much smaller wear debris forms a thick transfer layer. It is the interaction of this transfer debris layer with the counterface that controls the friction behavior during wear tests, as discussed before. No delamination of the coating can be observed. In the middle of the wear track the transfer material is well adhered to the disc surface. Some wear debris outside the sliding region could been observed.

EDX spectroscopy measurements were taken concurrent with SEM to identify the worn material elemental composition after the tests. The point shoot analysis of free debris, depicted by the points on figure G.3 revealed that the material consists of high amount of carbon 97,3 to $98,9 \%$, Si up to $2.6 \%$ and Fe. The material at the points 4 and 5 contains also $\mathrm{O}_{2} 26 \%$ and $\mathrm{Fe} 10.7 \%$, possibly a corrosion product. The zoom-in image on the worn spot (the white circle) is shown in figure G.3b. At the point 1, $64 \% \mathrm{~W}$ and $31 \% \mathrm{C}$ was found the rest is Ti, Cr, Fe. The point 2 contains $21 \% \mathrm{~W}$ and $77 \% \mathrm{C}$, the rest consists of $\mathrm{Ti}$ and Fe. Titanium is used as an interlay to improve the coating's adhesion. The other metallic elements like tungsten are used as an alloying element in DLC coatings. 
a)

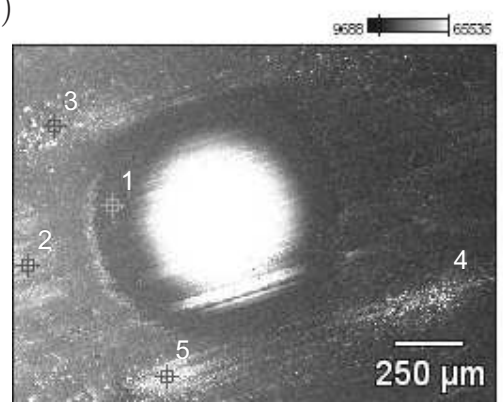

b)

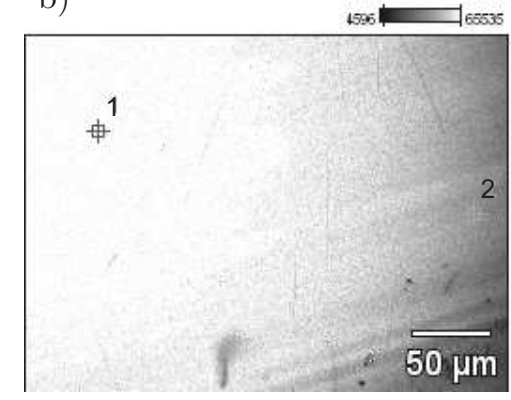

Figure G.3: a) SEM+EDX analysis on the pin surface after the test ld8vac, coating $\mathrm{H} 1$, in vacuum and b) zoom-in on the worn spot

a)

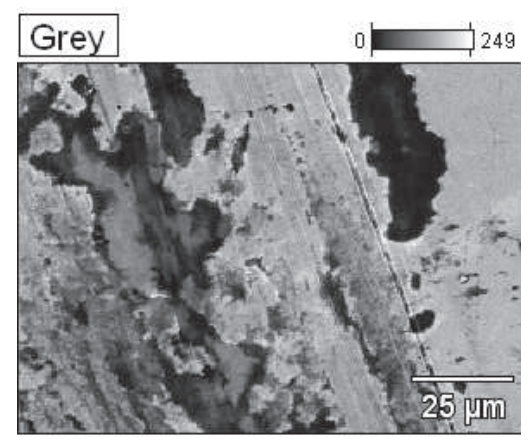

b)

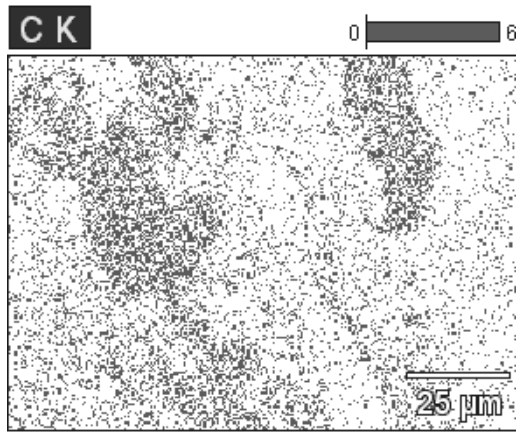

Figure G.4: SEM+EDX analysis on the disc wear track after the test ld8vac, coating $\mathrm{H} 1$, in vacuum a) and the carbon concentration map b) 
Figure G.4a shows SEM image of the disc wear track from test ld8vac. The carbon concentration given on the image G.4b corresponds well to the black areas in figure G.4a. This finding confirms that this is the transfer material from the pin surface. 


\section{Nomenclature}

\section{Greek symbols}

$\begin{array}{ll}\boldsymbol{\epsilon} & \text { strain tensor } \\ \boldsymbol{\Phi} & \text { contact function } \\ \boldsymbol{\sigma} & \text { Cauchy stress tensor } \\ \boldsymbol{\tau} & \text { contact stress tensor } \\ \alpha & \text { thermal expansion coefficient } \\ \delta & \text { admissible variation field } \\ \Delta & \text { difference; increment } \\ \eta & \text { gas viscosity } \\ \kappa & \text { heat transfer coefficient on the contact interface } \\ \Lambda & \text { bearing number } \\ \lambda & \text { failure rate } \\ \lambda & \text { thermal conductivity } \\ \mu & \text { coefficient of friction } \\ \Omega & \text { analysis domain, volume } \\ \rho & \text { density } \\ \tau & \text { gas shear stress }\end{array}$

\section{Roman symbols}

$\begin{array}{ll}\mathbf{D} & \text { rate of deformation } \\ \mathbf{d} & \text { surface relative velocity } \\ \mathbf{E} & \text { elasticity tensor } \\ \mathbf{f} & \text { force vector in a discretized system } \\ \mathbf{F} & \text { force vector } \\ \mathbf{I} & \text { second-order unit tensor } \\ \mathbf{K} & \text { stiffness matrix } \\ \mathbf{K} & \text { thermal material tensor } \\ \mathbf{L} & \text { velocity gradient tensor } \\ \mathbf{q} & \text { heat flow } \\ \mathbf{t} & \text { traction on a surface }\end{array}$




\begin{tabular}{|c|c|}
\hline $\mathbf{u}$ & displacement vector \\
\hline $\mathbf{v}$ & velocity vector \\
\hline $\mathbf{w}$ & vector of weight functions \\
\hline $\mathbf{x}$ & current cartesian coordinates \\
\hline $\mathbf{X}$ & reference cartesian coordinates \\
\hline$a$ & acceleration \\
\hline$B$ & bulk modulus \\
\hline$c$ & initial piston/cylinder clearance \\
\hline$c_{p}$ & heat capacity \\
\hline$d$ & sliding distance \\
\hline$D$ & diameter \\
\hline$E$ & elastic modulus \\
\hline$f$ & frequency \\
\hline$F_{L}$ & lift force \\
\hline$g$ & contact gap \\
\hline$G$ & shear modulus \\
\hline$h$ & gas film thickness \\
\hline$H$ & material hardness \\
\hline$I$ & moment of inertia \\
\hline$k$ & wear rate coefficient \\
\hline$L$ & piston length \\
\hline$m$ & mass \\
\hline$M$ & moment \\
\hline$N$ & interpolation function \\
\hline$P$ & power \\
\hline$p$ & pressure \\
\hline$Q$ & cycle averaged gas leakage \\
\hline$q$ & gas flow rate \\
\hline$R$ & radius \\
\hline$S$ & piston stroke \\
\hline$T$ & temperature \\
\hline$t$ & time \\
\hline$v$ & velocity \\
\hline$V$ & volume \\
\hline$W$ & work \\
\hline$z_{c g}$ & piston center of gravity \\
\hline
\end{tabular}

\section{Operators}

$\begin{array}{ll}\mathbf{x}^{\mathrm{T}} & \text { transpose of } \mathbf{x} \\ \dot{x} & \text { time derivative of } \mathrm{x} \\ \nabla x & \text { gradient of } \mathrm{x}\end{array}$




\section{General superscripts and subscripts}
(.) $)^{A, B} \quad$ body A, B
(.) ${ }^{A} \quad$ ambient
$(.)^{\text {el }} \quad$ elastic part
$(.)^{\text {th }} \quad$ thermal part
$(.)^{\mathrm{p}} \quad$ plastic part
$(.)_{\mathrm{p}} \quad$ piston
$(.)^{\mathrm{M}} \quad$ mechanical part
$(.)^{\mathrm{T}} \quad$ thermal part
(.) 0 initial or nominal state
$(.)_{\mathrm{C}} \quad$ cold
$(.)_{\mathrm{C}} \quad$ contact
(.) $)_{\mathrm{S}}$ surface
(.) crank

$\begin{array}{ll}\text { Abbreviations } \\ \text { ALE } & \text { arbitrary Lagrangian Eulerian } \\ \text { BC } & \text { boundary conditions } \\ \text { BL } & \text { boundary lubrication } \\ \text { BTC } & \text { bottom dead center } \\ \text { CoF } & \text { coefficient of friction } \\ \text { COP } & \text { coefficient of performance } \\ \text { CSM } & \text { continuous stiffness measurement } \\ \text { CVD } & \text { chemical vapour deposition } \\ \text { DLC } & \text { diamond like carbon } \\ \text { EDX } & \text { energy-dispersive X-ray } \\ \text { FDM } & \text { finite difference method } \\ \text { FEM } & \text { finite element method } \\ \text { FFL } & \text { full film lubrication } \\ \text { HTS } & \text { high temperature superconductivity } \\ \text { ML } & \text { mixed lubrication } \\ \text { MTTF } & \text { mean time to failure } \\ \text { PC } & \text { personal computer } \\ \text { PCA } & \text { piston cylinder assembly } \\ \text { PVD } & \text { physical vapour deposition } \\ \text { RPM } & \text { revolutions per minute } \\ \text { RT } & \text { room temperature } \\ \text { sec } & \text { seconds } \\ \text { SEM } & \text { scanning electron microscopy } \\ \text { SPM } & \text { secondary piston motion } \\ \text { TDC } & \text { top dead center } \\ \text { UL } & \text { updated Lagrangian } \\ \text { e.g. } & \text { exempli gratia (for example) } \\ & \end{array}$


i.e. id est (that is) 


\section{Acknowledgments}

This project has been sponsored by the STW foundation, the research was carried out at the University of Twente in the three research groups: Applied Mechanics, Thermal Engineering and Surface Engineering and Tribology.

I gratefully acknowledge all the people who have contributed in one way or another to this work.

First of all I would like to thank my promotor, Prof. Han Huétink for giving me the opportunity to work in the DiekA group. I am grateful for your guidance and scientific support. You gave me possibilities to develop myself, both scientifically and personally. This thesis owes much to my daily supervisor Dr. ir. Bert Geijselaers. Bert, I would like to thank you for your critical look at my progress and constructive criticism. I am grateful for your support, helpful discussions and for always being available when I needed you.

When I look back I realize that I have been particulary fortunate that I had the opportunity to do my master project in the DiekA group. Although forming processes was not my preferred specialization, the application projects and analysis of heat transfer in machine components with DiekA gave us the idea of preparing a research project proposal. Recognizing the need for Dutch piston compressor producers and their support made this project come true. Thanks to all who supported me in preparing this proposal.

This research was carried out within two parallel running projects. Uros Lekić worked under supervision of Jim Kok. Thank you for your contribution to this work. Part of my research was done in the Tribology Group. I thank Matthijn de Rooij for numerous advice concerning coatings and tribology.

Special thanks go to the industrial partners who joined this project for their support and encouragement. I would like to acknowledge Erik Haenen (Stirling Cryogenics BV). Thanks Erik for your enthusiasm about this project and valuable inputs. I am grateful to you for reading my thesis thoroughly and sharing your comments. Additionally, Tonny Benschop (Thales Cryogenics BV), Gorge Bon (GEA Grasso BV), Harry Slaghuis (Noble Products Twente BV), Semfira Stoye (Noble Products Germany), Roland Groenen (Bekaert Advanced Coatings). 
I owe my appreciation to the following collaborators who helped us to build the experimental set-ups and supported us when performing the experiments. Particulary, I would like to mention Erik de Vries for building the vacuum tester and for the many friction measurements he carefully carried out and other various supports and discussions regarding measurement techniques. Henk-Jan Moed for his support during constructing the helium installation and instrumentation of the gas spring. Willy Kerve for making parts and preparing samples for the experiments. Special thanks go to Wim ten Heggeler (TenHeggeler Machine Fabriek) and his co-workers for professional support and understanding manufacturing components for the gas spring set-up. Thanks to Laura Vargas from the Production Technology Group for the beautiful SEM pictures. Many thanks also go to Dr Jacek Grabarczyk from the Technical University of Łódź for performing the nano-indentation measurements.

I would like to acknowledge Han Huétink, Bert Geijselaers and Matthijn de Rooij for carefully reading my thesis and helping me with improving its content considerably.

I would like to express my gratitude to all permanent members of the group Ton van den Boogaard, Harm Wisselink, Timo Meinders, Nico van Vliet, Herman van Corbach, Wilko Emmens and former and present researchers at the group for your time and valuable suggestions. The positive and open working environment was very encouraging and motivating. Working with DiekA code would simply not be possible without Harm, Timo, Nico and Herman and their support is greatly acknowledged. My thanks go to Tania Gerrits and Debbie Vrieze-Zimmerman van Woesik for helping me with many administrative and other personal questions during my project. Additionally, the efforts of Vivien Cook in correcting the English language are appreciated.

I also would like to thank all colleagues who I had the chance to meet from the Applied Mechanics section providing a wonderful social environment. In particular, many thanks to Didem Akcay and Semih Perdahcioğlu, Bert Koopman, Igor Burchitz, Marieke Hannink, Emre Dikmen, Wissam Assaad, Ashraf Hadoush, Maarten van Riel, Srihari Kurukuri, Wouter Quak, Muhammad Niazi and Johan Hol.

Special thanks to my parents for continuous encouragement, confidence in me and support in different ways. Many thanks to all my family in Poland for showing interests in my work and for motivation.

Szczególne podziȩkowania moim rodzicom za nieustanne wsparcie, zaufanie oraz pomoc wszelkiego rodzaju. Wiele podziȩkowań catej moje rodzinie w Polsce za okazane zainteresowanie moja praca i motywacje.

Finally I want to thank my wife Iza for her love and trust. I received indispensable support from you during this time. Thank you. 


\section{References}

[1] E.A. Atkinson. An introduction to numerical analysis. John Wiley and Sons, 1978.

[2] P.B. Bailey, M.W. Dadd, J.S Reed, and C.R. Stone. Gas spring losses in linear clearance seal compressors. In S.D. Miller and R.G. Ross, editors, International Cryocooler Conference, 2007.

[3] K.D. Beaty. Long-life engine design. Technical report.

[4] B. Bhushan. Modern tribology handbook. CRC Press LLC, 2001.

[5] E. Broszeit, C. Friedrich, and G. Berg. Deposition, properties and applications of PVD CrxN coatings. Surface and Coatings Technology, 115(1):9-16, 1999.

[6] Hauzer Techno Coating BV. http://www.hauzertechnocoating.com.

[7] B. Carleer. Finite element analysis of deep drawing. PhD thesis, University of Twente, 1997.

[8] Bekaert Advanced Coating. www.bekaert.com.

[9] V.N. Constantinescu, A. Nica, i M.D. Pascovic, G. Ceptureanu, and S. Nedelcu. Sliding bearings. Allerton Press Inc./ New York, 1985.

[10] V.N. Constantinscu. Gas lubrication. The American Society of Mechanical Engineers, 1969.

[11] R.D. Cook and Plesha M.E. Concept and application of finite element analysis. John Wiley and Sons, 2001.

[12] Thales Cryogenics. http://www.thales-cryogenics.com/.

[13] Thales Cryogenics. Personal communication on existing coatings used in cryocoolers. 
[14] H. Czichos. Tribology: A System Approach to the Science and Technology of Friction, Lubrication and Wear. Elsevier Scientific Publishing Company, Amsterdam, Oxford, New York, 1978., 1978.

[15] D. Dascalescu, K. Polychronopoulou, and A. A. Polycarpou. The significance of tribochemistry on the performance of PTFE-based coatings in co2 refrigerant environment. Surface and Coatings Technology, 204(3):319-329, 2009.

[16] N. G. Demas and A. A. Polycarpou. Tribological performance of PTFE-based coatings for air-conditioning compressors. Surface and Coatings Technology, 203(3-4):307-316, 2008.

[17] D.W. Dickey and Ch.D. Wood. Analitical and experimental investigation of ringless piston concept. Technical report, Gas Research Institute, 1988.

[18] M.F. Doerner and W.D. Nix. Stresses and deformation processes in thin films on substrates. CRC critical reviews in solid state and materials sciences, 14(3):225$268,1988$.

[19] C. Donnet, M. Belin, J. C. Auge, J. M. Martin, A. Grill, and V. Patel. Tribochemistry of diamond-like carbon coatings in various environments. Surface and Coatings Technology, 68-69(C):626-631, 1994.

[20] C. Donnet and A. Erdemir. Tribology of Diamond-Like Carbon Films. Springer, 2008.

[21] C. Donnet, T. Le Mogne, L. Ponsonnet, M. Belin, A. Grill, V. Patel, and C. Jahnes. The respective role of oxygen and water vapor on the tribology of hydrogenated diamond-like carbon coatings. Tribology Letters, 4(3-4):259-265, 1998.

[22] A. Erdemir. U.S patent 6.548.173 b2.

[23] A. Erdemir. The role of hydrogen in tribological properties of diamond-like carbon films. Surface and Coatings Technology, 146-147:292-297, 2001.

[24] A. Erdemir. Genesis of superlow friction and wear in diamondlike carbon films. Tribology International, 37(11-12 SPEC.ISS.):1005-1012, 2004.

[25] A. Erdemir, C. Bindal, G. R. Fenske, C. Zuiker, and P. Wilbur. Characterization of transfer layers forming on surfaces sliding against diamond-like carbon. Surface and Coatings Technology, 86-87(1 -3 pt 2):692-697, 1996.

[26] A. Erdemir, C. Bindal, J. Pagan, and P. Wilbur. Characterization of transfer layers on steel surfaces sliding against diamond-like hydrocarbon films in dry nitrogen. Surface and Coatings Technology, 76-77(PART 2):559-563, 1995.

[27] A. Erdemir and C. Donnet. Tribology of diamond-like carbon films: Recent progress and future prospects. Journal of Physics D: Applied Physics, 39(18), 2006. 
[28] A. Erdemir and G. R. Fenske. Tribological performance of diamond and diamondlike carbon films at elevated temperatures. Tribology Transactions, 39(4):787-794, 1996.

[29] I. Etsion and K. Gommed. Improved design with noncylindrical profiles of gaslubricated ringless pistons. Journal of Tribology, 117(1):143-147, 1995.

[30] K. Farrell, E. D. Specht, J. Pang, L. R. Walker, A. Rar, and J. R. Mayotte. Characterization of a carburized surface layer on an austenitic stainless steel. Journal of Nuclear Materials, 343(1-3):123-133, 2005.

[31] J. Fontaine, T. Le Mogne, J. L. Loubet, and M. Belin. Achieving superlow friction with hydrogenated amorphous carbon: Some key requirements. Thin Solid Films, 482(1-2):99-108, 2005.

[32] J. Fontaine, J. L. Loubet, T. Le Mogne, and A. Grill. Superlow friction of diamond-like carbon films: A relation to viscoplastic properties. Tribology Letters, 17(4):709-714, 2004.

[33] C. A. Freyman, Y. Chen, and Y. W. Chung. Synthesis of carbon films with ultra-low friction in dry and humid air. Surface and Coatings Technology, 201(12):164-167, 2006.

[34] J. A. Gatowski, M. K. Smith, and A. C. Alkidas. An experimental investigation of surface thermometry and heat flux. Experimental Thermal and Fluid Science, $2(3): 280-292,1989$.

[35] J.A. Gatowski, M.K. Smith, and A.C. Alkidas. Experimental investigation of surface thermometry and heat flux. Experimental Thermal and Fluid Science, pages 280-292, 1992.

[36] G. Gille and B. Rau. Buckling instability and adhesion of carbon layers. Thin Solid Films, 120(2):109-121, 1984.

[37] K. Gommed and I. Etsion. Dynamic analysis of gas lubricated reciprocating ringless pistons. basic modeling. Journal of Tribology, 115(2):207-213, 1993.

[38] K. Gommed and I. Etsion. Parametric study of the dynamic performance of gas lubricated ringless pistons. Transactions - ASME: Journal of Tribology, 116(1):63-69, 1994.

[39] A. Grill. Diamond-like carbon: State of the art. Diamond and Related Materials, 8(2-5):428-434, 1999.

[40] W.A. Gross, editor. Theory of lubrication: with applications to liquid- and gasfilm lubrication. Stanford, CA : Stanford University Press, 1962.

[41] J. Guttler and J. Reschke. Metal-carbon layers for industrial application in the automotive industry. Surface and Coatings Technology, 60(1-3):531-535, 1993. 
[42] R.B. Haber and B.H. Harjandja. An Eulerian-Lagrangian finite element approach to large deformation frictional contact. Computers and Structures, 30(1-3):193$201,1985$.

[43] T. Hejwowski and A. Weroński. The effect of thermal barrier coatings on diesel engine performance. Vacuum, 65(3-4):427-432, 2002.

[44] S. Hogmark and P. Hedenqvist. Tribological characterization of thin, hard coatings. Wear, 179(1-2):147-154, 1994.

[45] H. Holleck, M. Lahres, and P. Woll. Multilayer coatings-influence of fabrication parameters on constitution and properties. Surface and Coatings Technology, 41(2):179-190, 1990.

[46] J. Huétink. On the simulation of thermomechanical forming processes. PhD thesis, University of Twente, 1986.

[47] J. Huétink and H. G. Mooi. Application of numerical simulation in design and control of forming processes. Journal De Physique, 3(7 pt 2):1129-1134, 1993.

[48] J. Huétink, P. T. Vreede, and J. Van Der Lugt. Progress in mixed EulerianLagrangian finite element simulation of forming processes. International Journal for Numerical Methods in Engineering, 30(8):1441-1457, 1990.

[49] K.L. Johnson. Contact mechanics. Cambridge University Press, 1989.

[50] A. Matthews K. Holmberg, editor. Coatings tribology, volume 28 of Tribology series. Elsevier, 1994.

[51] K. Kato, N. Umehara, and K. Adachi. Friction, wear and N2-lubrication of carbon nitride coatings: A review. Wear, 254(11):1062-1069, 2003.

[52] A. Kaźmierczak. The new ring seal of the combustion engines and its surface free energy. Industrial Lubrication and Tribology, 56(1):6-13, 2004.

[53] P.J. Kerney and M. Nisenoff. Workshop on cryogenic packaging of high temperature superconducting (HTS) electronic devices. Technical report, 1995.

[54] J.W.L. Kohler. The stirling refrigeration cycle. Scientific American, 212(119127), 1965.

[55] L.G.M. Koop. Free floating piston, case history of revamp of existing compressors, 2005.

[56] A. A. Kornhauser. Dynamic modeling of gas springs. Journal of Dynamic Systems, Measurement and Control, Transactions of the ASME, 116(3):414-418, 1994.

[57] H.H. van den Kroonenberg and F.J. Siers. Methodisch ontwerpen. Educaboek, Culemborg, 1992. 
[58] S. Kwaśniowski, Z.J. Sroka, and W. Zabłocki. Modelling Thermal Loads in Combustion Engine Components [in Polish]. Wroclaw University of Technology Publishing House, Wroclaw, 1999.

[59] U. Lekić and J.B.W. Kok. Heat and fluid flows in gas spring. 2009.

[60] U. Lekić and J.B.W. Kok. Heat transfer and fluid flows in gas springs. The Open Thermodynamics Journal, 4:13-26, 2010.

[61] D.F. Li, S.M. Rohde, and H.A. Ezzat. Automotive piston lubrication model. ASLE transactions, 26(2):151-160, 1983.

[62] E. Liu, X. Shi, H. S. Tan, L. K. Cheah, Z. Sun, B. K. Tay, and J. R. Shi. The effect of nitrogen on the mechanical properties of tetrahedral amorphous carbon films deposited with a filtered cathodic vacuum arc. Surface and Coatings Technology, 120-121:601-606, 1999.

[63] Y. Liu, A. Erdemir, and E. I. Meletis. A study of the wear mechanism of diamondlike carbon films. Surface and Coatings Technology, 82(1-2):48-56, 1996.

[64] K. Miyoshi, F.S. Honecy, P.B. Abel, S.V. Pepper, T. Spalvins, and D.R. Wheeler. Vacuum $\left(10^{-9}\right.$ Torr $)$ friction apparatus for determining friction and endurance life of $\mathrm{MoS}_{x}$ films. STLE Tribology Transactions, 36(3):351-358, 1993.

[65] Z. P. Mourelatos. Gas lubrication of a ringless piston in an internal combustion engine under dynamic loading. Journal of Tribology, 110(4):718-726, 1988.

[66] W. C. Oliver and G. M. Pharr. Improved technique for determining hardness and elastic modulus using load and displacement sensing indentation experiments. Journal of Materials Research, 7(6):1564-1580, 1992.

[67] P. Owczarek, M. de Rooij, and E. de Vries. Tribological evaluation of DLC coatings in an inert atmosphere. In Seals and sealing technology of machines and devices, Kudowa Zdrój, 2010.

[68] P. Owczarek and H.J.M. Geijselaers. Analysis of thermo-mechanical distortions in sliding components - an ALE approach. Proceedings of the STLE/ASME International Joint Tribology Conference, 2008.

[69] P. Owczarek and H.J.M. Geijselaers. Analysis of secondary piston motion of a gas lubricated compressor. In Seals and sealing technology of machines and devices, Kudowa Zdrój, 2010.

[70] R.H. Pasaribu. Friction and wear of zirconia and alumina ceramics doped with CuO. PhD thesis, University of Twente, 2005.

[71] A.D. Rietman. Numerical analysis of inhomogenieous deformation in plane strain compression. PhD thesis, University of Twente, 1999. 
[72] J. Robertson. Diamond-like amorphous carbon. Technical report, Engineering Department, Cambridge University, 2002.

[73] H. Ronkainen, S. Varjus, and K. Holmberg. Friction and wear properties in dry, water- and oil-lubricated DLC against alumina and DLC against steel contacts. Wear, 222(2):120-128, 1998.

[74] J. C. Sánchez-López, M. Belin, C. Donnet, C. Quiro's, and E. Elizalde. Friction mechanisms of amorphous carbon nitride films under variable environments: A triboscopic study. Surface and Coatings Technology, 160(2-3):138-144, 2002.

[75] S. Sasaki. The effects of the surrounding atmosphere on the friction and wear of alumina, zirconia, silicon carbide and silicon nitride. Wear, 134(1):185-200, 1989.

[76] C. Strondl, N. M. Carvalho, J. Th M. De Hosson, and G. J. Van der Kolk. Investigation on the formation of tungsten carbide in tungsten-containing diamond like carbon coatings. Surface and Coatings Technology, 162(2-3):288293, 2003.

[77] I. Sugimoto and S. Miyake. High lubrication performance of tribologically oriented fluoropolymer molecules analyzed by polarized infrared microspectroscopy. Journal of Applied Physics, 67(9):4083-4089, 1990.

[78] Bodycote Metal Technology. Promotional information on the Kolsterising metal treatment, Bodycote Metal Technology, 2005.

[79] H. J. M. ter Brake and G. F. M. Wiegerinck. Low-power cryocooler survey. Cryogenics, 42(11):705-718, 2002.

[80] H.J.M. ter Brake. Cryogenic systems for superconducting devices. In H. Weinstock, editor, Applications of Superconductivity, pages 561-639. Kluwer Academic Publishers, 2000.

[81] J. v/d Lugt. A finite element method for the simulation of thermo-mechanical contact problems in forming processes. PhD thesis, University of Twente, 1988.

[82] P.T. Vreede. A finite element method for the simulations of 3-dimensional sheet metal forming. PhD thesis, University of Twente, 1990.

[83] J. Wang and M. S. Gadala. Formulation and survey of ALE method in nonlinear solid mechanics. Finite Elements in Analysis and Design, 24(4):253-269, 1997.

[84] N. Wang and Ch. Chang. An application of Newton's method to the lubrication analysis of air-lubricated bearings. Tribilogy Transactions, 42(2):419-424, 1999.

[85] Y. Wang and S. C. Tung. Scuffing and wear behavior of aluminum piston skirt coatings against aluminum cylinder bore. Wear, 225-229(PART II):1100-1108, 1999. 
[86] H.H. Wisselink. Analysis of guillotining and slitting. PhD thesis, University of Twente, 2000.

[87] A. Yamaguchi. Motion of the piston in piston pumps and motors (Experiments and theoretical discussion). JSME International Journal, Series B: Fluids and Thermal Engineering, 37(1):83-88, 1994.

[88] D. Zhu, Y-Z Hu, H.S. Cheng, T. Arai, and K. Hamai. Numerical analysis for piston skirts in mixed lubrication - Part II: Deformation considerations. Journal of Tribology, 115(1):125-133, 1993.

[89] O.C. Zienkiewicz and R.L. Taylor. The finite element method. Elsevier Butterworth-Heinemann, 2005.

[90] J. Zuk. Fundamentals of fluid sealing. Lewis Research Center Cleveland, Ohio, 1976. 